\title{
SELECTED HYDROLOGIC DATA FOR CACHE VALLEY, UTAH AND IDAHO, 1969-91
}

By D. Michael Roark and Karen M. Hanson

UTAH HYDROLOGIC-DATA REPORT NO. 48

U.S. GEOLOGICAL SURVEY

Open-File Report 92-173

Prepared in cooperation with the

UTAH DEPARTMENT OF NATURAL RESOURCES,

DIVISION OF WATER RESOURCES

DIVISION OF WATER RIGHTS

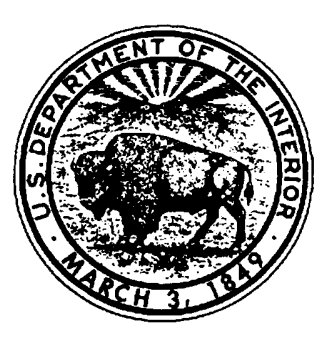

Salt Lake City, Utah 


\title{
U.S. DEPARTMENT OF THE INTERIOR
}

\section{MANUEL LUJAN, JR., Secretary}

\author{
U.S. GEOLOGICAL SURVEY \\ Dallas L. Peck, Director
}

For additional information write to:

District Chief

U.S. Geological Survey, WRD

Room 1016 Administration Building

1745 West 1700 South

Salt Lake City, Utah 84104
Copies of this report can be purchased from:

U.S. Geological Survey

Books and Open-File Reports Section

Federal Center

Box 25425

Denver, Colorado 80225 


\section{CONTENTS}

Page

Introduction

1

References cited.

1

\section{ILLUSTRATIONS}

(Plate is in pocket)

Plate 1. Map showing location of selected hydrologic-data sites, Cache Valley,

Utah and Idaho, 1969-91.

Figure 1. Diagram showing numbering systems used in this report for

hydrologic-data sites in Utah and Idaho

\section{TABLES}

Table 1. Records of selected wells........................................................... 4

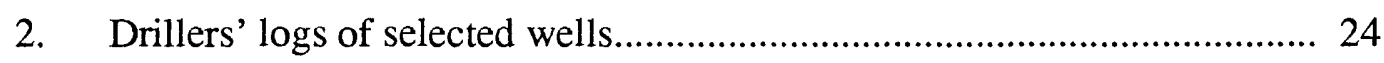

3. Water levels in selected wells ........................................................... 29

4. Discharge of selected flowing wells .................................................. 55

5. Temperature and specific conductance of water from selected wells........ 57

6. Records of selected springs ............................................................6 60

7. Field measurements of discharge, specific conductance, and water temperature at selected surface-water sites 


\section{CONVERSION FACTORS, VERTICAL DATUM, AND ABBREVIATED WATER-QUALITY UNITS}

\section{Multiply}

acre

foot

cubic foot per second

gallon per minute

inch

mile

square mile
By

To obtain

$\begin{array}{cl}0.4047 & \text { hectare } \\ 4,047 & \text { square meter } \\ 0.3048 & \text { meter } \\ 0.02832 & \text { cubic meter per second } \\ 0.06308 & \text { liter per second } \\ 25.4 & \text { millimeter } \\ 0.0254 & \text { meter } \\ 1.609 & \text { kilometer } \\ 2.59 & \text { square kilometer }\end{array}$

Water temperature is given in degrees Celsius $\left({ }^{\circ} \mathrm{C}\right)$, which can be converted to degrees Fahrenheit $\left({ }^{\circ} \mathrm{F}\right)$ by the following equation:

$$
{ }^{\circ} \mathrm{F}=1.8\left({ }^{\circ} \mathrm{C}\right)+32 .
$$

Sea level: In this report, "sea level" refers to the National Geodetic Vertical Datum of 1929-a geodetic datum derived from a general adjustment of the first-order level nets of the United States and Canada, formerly called Sea Level Datum of 1929.

Specific conductance and water temperature are given only in metric units. Specific conductance is given in microsiemens per centimeter $(u \mathrm{~S} / \mathrm{cm})$ at 25 degrees Celsius. 


\title{
SELECTED HYDROLOGIC DATA FOR CACHE VALLEY, UTAH AND IDAHO, 1969-91
}

\author{
By D. Michael Roark and Karen M. Hanson
}

\section{INTRODUCTION}

This report contains hydrologic data collected in Cache Valley from 1969 to 1991. The report area is in north-central Utah and southeast Idaho, within the Basin and Range physiographic province described by Fenneman (1931), and includes about 660 square miles.

Most of the data in this report were collected by the U.S. Geological Survey in cooperation with the Utah Department of Natural Resources, Divisions of Water Resources and Water Rights. Some of the data collected before 1969 were previously published by McGreevy and Bjorklund (1970).

The purpose of this report is to provide hydrologic data for use by the general public and by officials managing the area's water resources, and to supplement interpretive reports for the area. Tables 1 to 7 contain selected well, spring, and surface-water data. The numbering systems used in this report for hydrologic-data sites are illustrated in figure 1. Hydrologic-data sites are shown on plate 1 .

These data could not have been collected without the cooperation of local residents and officials of irrigation companies and municipalities, that permitted access to their wells, springs, and canals to measure water levels in wells and flow in springs and canals. The Idaho Department of Water Resources also provided valuable assistance and data.

\section{REFERENCES CITED}

Fenneman, N.M., 1931, Physiography of the western United States: McGraw-Hill, New York, $534 \mathrm{p}$.

McGreevy, L.J., and Bjorklund, L.J., 1970, Selected hydrologic data, Cache Valley, Utah and Idaho: Utah Basic Data Release 21, $51 \mathrm{p}$. 
The system of numbering wells and springs in Utah is based on the cadastral land-survey system of the U.S. Government. The number, in addition to designating the well or spring, describes its position in the land net. The land-survey system divides the State into four quadrants separated by the Salt Lake Base Line and Meridian. These quadrants are designated by the uppercase letters A, B, C, and D, indicating the northeast, northwest, southwest, and southeast quadrants, respectively. Numbers designating the township and range, in that order, follow the quadrant letter, and all three are enclosed in parentheses. The number after the parentheses indicates the section and is followed by three letters indicating the quarter section, the quarter-quarter section, and the quarterquarter-quarter section, generally 10 acres $^{1}$ for regular sections. The lower case letters a, b, c, and d indicate, respectively, the northeast, northwest, southwest, and southeast quarters of each subdivision. The number after the letters is the serial number of the well or spring within the 10-acre tract. The letter $\mathrm{S}$ preceding the serial number designates a spring. The letter $\mathrm{W}$ following the serial number denotes a stream, and the letter $\mathrm{B}$ denotes a canal or ditch. Thus, (A-12-1)29cab-1 designates the first well constructed or visited in the NW $1 / 4 \mathrm{NE}^{1} / 4 \mathrm{SW}^{1} / 4$, section 29, T. 12 N., R. 1 E.

In Idaho, the well- and spring-numbering system is based on the cadastral land survey with reference to the Boise Base Line and Meridian. It is similar to the Utah system except that springs are not designated by the letter $\mathrm{S}$ and the quadrant letter is omitted; the townships are labeled $\mathrm{N}$ or $\mathrm{S}$ to designate north or south and the ranges are labeled $\mathrm{E}$ or $\mathrm{W}$ to designate east or west. The letter $\mathrm{W}$ following the serial number denotes a stream, and the letter B denotes a canal or ditch. Thus, 16S 40E 29CCB1 is the first well visited in the $\mathrm{NW}^{1} / 4 \mathrm{SW}^{1} / 4$ $\mathrm{SW}^{1 / 4} 4$, section 29, T. $16 \mathrm{~S}$., R. $40 \mathrm{E}$.

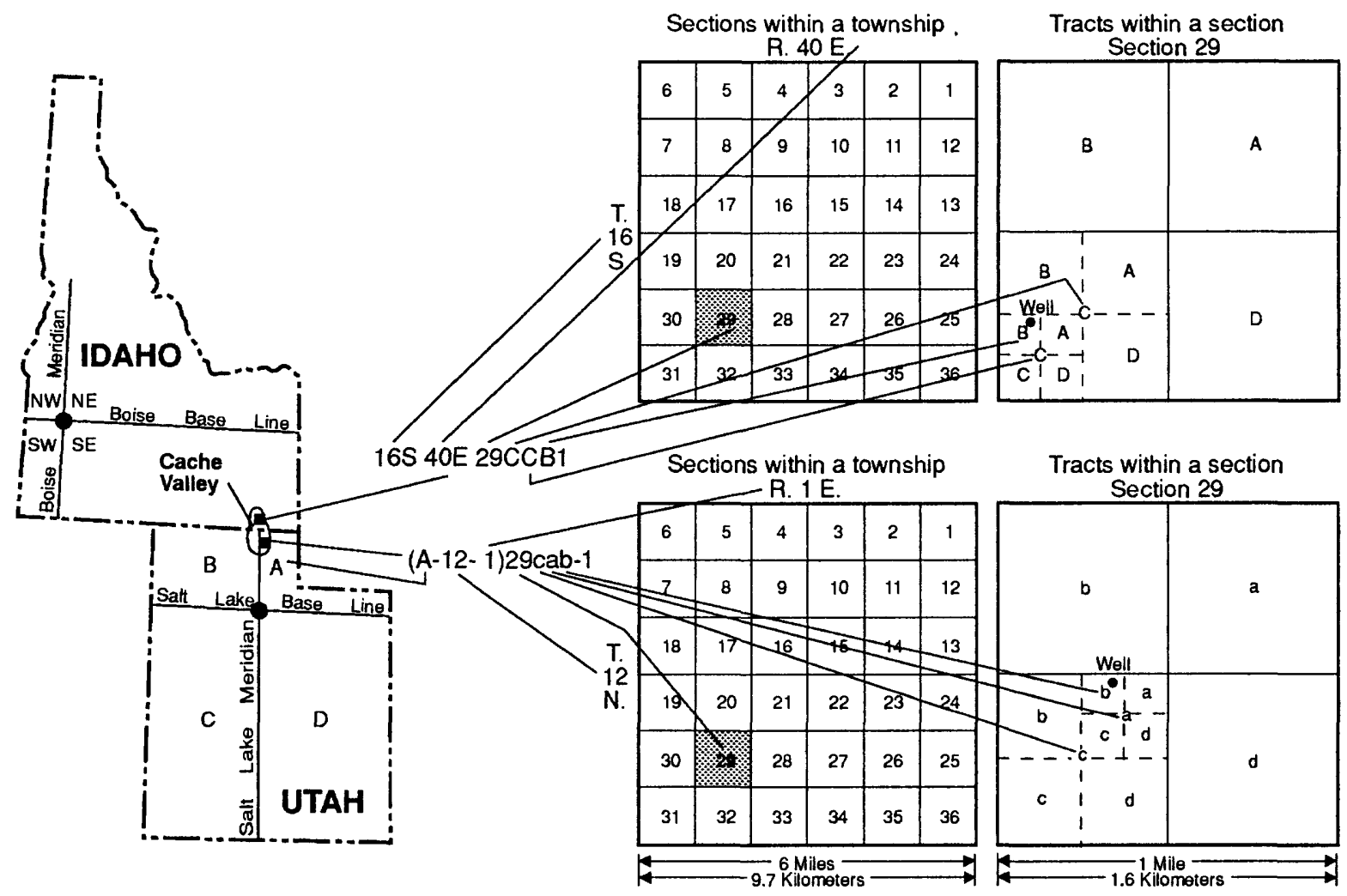

${ }^{1}$ Although the basic land unit, the section, is theoretically 1 square mile, many sections are irregular. Such sections are subdivided into
10 -acre tracts, generally beginning at the southeast corner, and the surplus or shortage is taken up in the tracts along the north and west
sides of the section.

Figure 1. Numbering systems used in this report for hydrologic-data sites in Utah and Idaho. 


\section{Table 1.-Records of \\ [- indicates no}

Location: See page 2 for an explanation of the numbering systems for hydrologic-data sites.

Owner or user: Refers to last known owner or user.

Use of water: H, domestic or household; P, public supply; U, unused; I, irrigation; S, stock; Q, aquaculture; T, Depth of well: Greatest depth drilled or greatest depth sounded.

Casing: Diameter: reported from driller's log or measured in the field. Finish: O, open ended; P, perforated; $S$, feet below the landsurface, if known, and questioned (?) if extent of perforated or screened interval is Altitude of land surface is given in feet above sea level.

Water level is given in feet and decimal fractions. Measured except where noted $\mathrm{R}$, reported.

Yield: Rate: gal/min, gallons per minute; $\mathrm{F}$, natural flow; $\mathrm{P}$, pumped.

Water-quality parameters: ${ }^{\circ} \mathrm{C}$, degrees Celsius; $\mu \mathrm{S} / \mathrm{cm}$, microsiemens per centimeter at 25 degrees Celsius.

Other data available: $\mathrm{L}$, driller's $\log$ (table 2); W, water-level measurements (table 3); C, temperature and

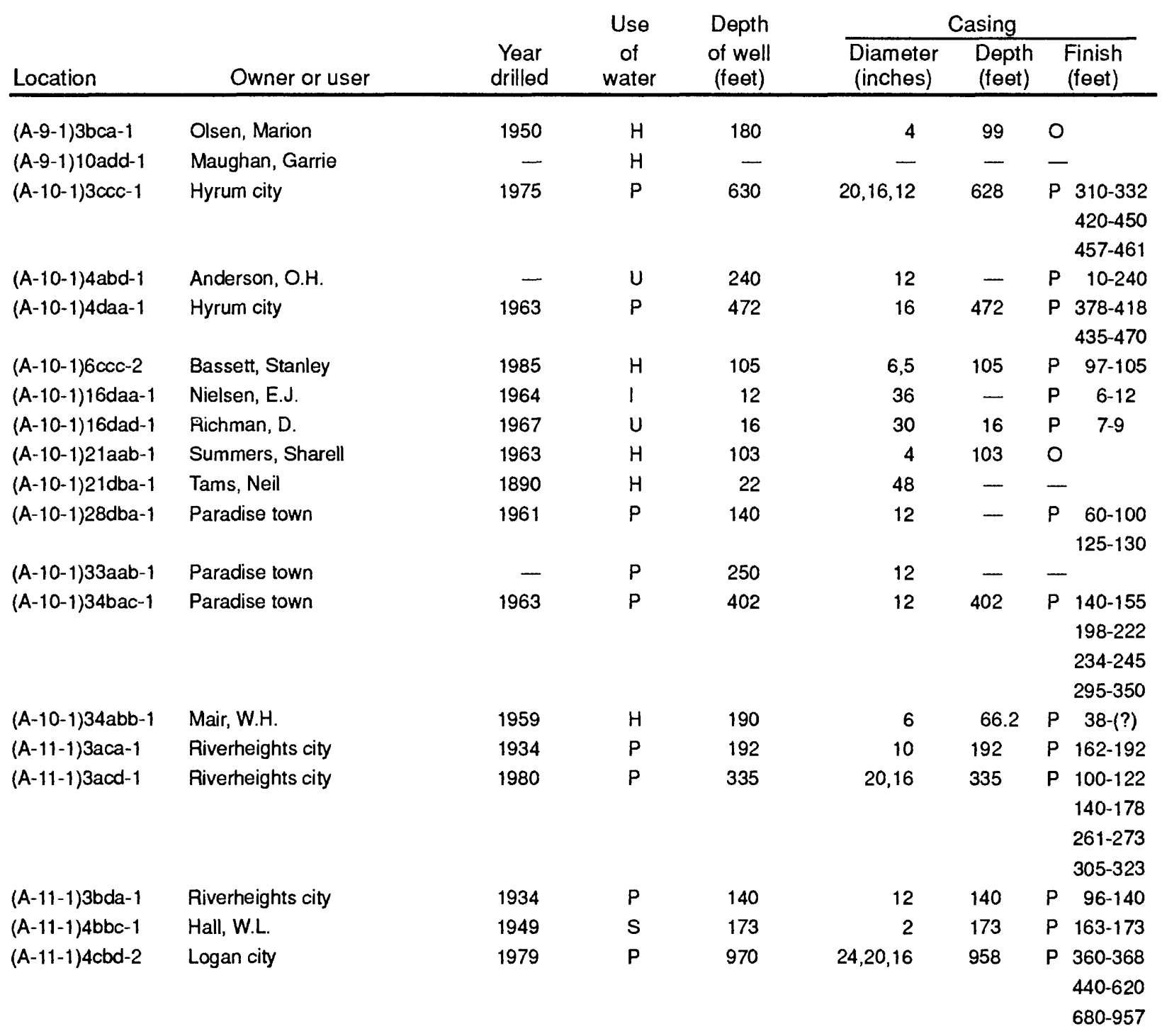


selected wells

data available]

institutional; $\mathrm{N}$, industrial; $\mathrm{C}$, emergency supply; $\mathrm{D}$, dewater.

screened; W, walled; H, horizontal gallery; $\mathrm{T}$, sand point. Upper and lower limits of perforations or screen given in unknown.

specific conductance of water (table 5); D, well-discharge measurements (table 4).

\begin{tabular}{|c|c|c|c|c|c|c|c|c|}
\hline \multirow{4}{*}{$\begin{array}{c}\text { Altitude } \\
\text { of land } \\
\text { surface } \\
\text { (feet) }\end{array}$} & \multicolumn{2}{|c|}{ Water level } & & & \multirow{2}{*}{\multicolumn{3}{|c|}{ Water-quality parameters }} & \multirow{4}{*}{$\begin{array}{c}\text { Other } \\
\text { data } \\
\text { available }\end{array}$} \\
\hline & \multirow{3}{*}{$\begin{array}{c}\text { Above (-) } \\
\text { or below } \\
\text { land surface } \\
\text { (feet) }\end{array}$} & \multirow[b]{3}{*}{ Date } & \multirow{2}{*}{\multicolumn{2}{|c|}{ Yield }} & & & & \\
\hline & & & & & \multirow[b]{2}{*}{$\begin{array}{c}\text { Temperature } \\
\left({ }^{\circ} \mathrm{C}\right)\end{array}$} & \multirow{2}{*}{$\begin{array}{c}\text { Specific } \\
\text { conductance } \\
(\mu \mathrm{S} / \mathrm{cm})\end{array}$} & \multirow[b]{2}{*}{ Date } & \\
\hline & & & $\begin{array}{c}\text { Rate } \\
\text { (gal/min) }\end{array}$ & Date & & & & \\
\hline 4,905 & 35.63 & $03-21-91$ & - & - & - & - & - & \\
\hline 5,000 & 49.45 & $03-06-91$ & - & - & - & - & - & \\
\hline 4,800 & - & - & - & - & 一 & - & - & L \\
\hline 4,705 & 10.26 & $03-20-91$ & - & - & - & - & - & \\
\hline 4,790 & - & - & - & - & - & - & - & \\
\hline 4,640 & 4.64 & $03-12-91$ & - & - & - & - & - & \\
\hline 4,800 & 50.04 & $03-06-91$ & - & - & 一 & - & - & \\
\hline 4,820 & 10.21 & $03-08-91$ & - & - & - & - & - & w \\
\hline 4,820 & 13.26 & $03-06-91$ & - & - & - & - & - & \\
\hline 4,830 & 18.23 & $03-21-91$ & - & - & - & - & - & \\
\hline 4,870 & - & - & - & - & - & - & - & \\
\hline 4,920 & - & - & - & - & - & - & - & \\
\hline 4,990 & - & - & - & - & - & - & - & \\
\hline 5,035 & 37.47 & $03-21-91$ & - & - & - & - & - & \\
\hline 4,600 & 172.00 & $04-03-91$ & - & - & - & - & - & \\
\hline 4,580 & 113.00 & $04-03-91$ & $1,040 P$ & $08-21-80$ & - & - & - & \\
\hline 4,540 & 52.00 & $04-03-91$ & - & - & - & - & - & \\
\hline 4,480 & 4.80 & $03-20-91$ & - & - & - & - & - & \\
\hline 4,485 & -4.9 & $03-21-91$ & - & - & - & - & - & $L$ \\
\hline
\end{tabular}


Table 1.-Records of

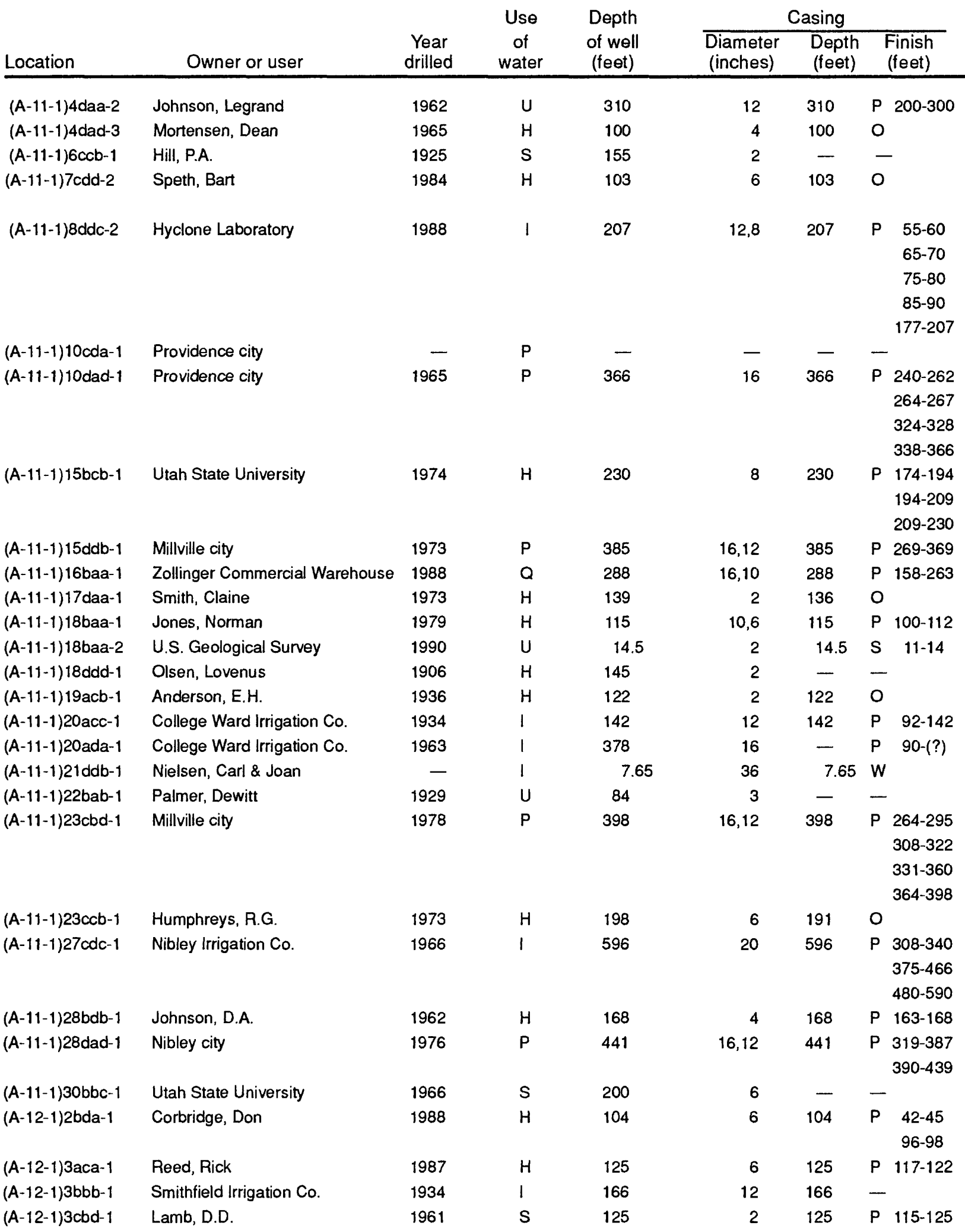




\begin{tabular}{|c|c|c|c|c|c|c|c|c|}
\hline \multirow{3}{*}{$\begin{array}{c}\text { Altitude } \\
\text { of land } \\
\text { surface } \\
\text { (feet) }\end{array}$} & \multicolumn{2}{|c|}{ Water level } & & & \multirow{2}{*}{\multicolumn{3}{|c|}{ Water-quality parameters }} & \multirow{3}{*}{$\begin{array}{c}\text { Other } \\
\text { data } \\
\text { available }\end{array}$} \\
\hline & \multirow{2}{*}{$\begin{array}{c}\text { Above (-) } \\
\text { or below } \\
\text { land surface } \\
\text { (feet) }\end{array}$} & \multirow[b]{2}{*}{ Date } & & & & & \\
\hline & & & $\begin{array}{c}\text { Yield } \\
\text { Rate } \\
\text { (gal/min) }\end{array}$ & $\overline{\text { Date }}$ & $\begin{array}{c}\text { Temperature } \\
\left({ }^{\circ} \mathrm{C}\right)\end{array}$ & $\begin{array}{c}\text { Specific } \\
\text { conductance } \\
(\mu \mathrm{S} / \mathrm{cm})\end{array}$ & Date & \\
\hline 4,500 & 24.75 & $03-08-91$ & - & - & - & - & - & W \\
\hline 4,505 & 16.91 & $03-08-91$ & - & - & - & - & - & \\
\hline 4,435 & -23.7 & $03-19-91$ & $4.3 \mathrm{~F}$ & $03-19-91$ & 11.0 & 450 & $03-19-91$ & \\
\hline \multirow[t]{2}{*}{4,435} & -27.2 & 03-19-91 & $50 \mathrm{~F}$ & $08-14-84$ & 10.5 & 445 & 03-19-91 & \\
\hline & & & $18.8 \mathrm{~F}$ & $03-19-91$ & & & & \\
\hline 4,480 & $6.9 \mathrm{R}$ & $03-04-91$ & - & - & - & - & - & \\
\hline 4,235 & 43.78 & $03-08-91$ & - & - & - & - & - & \\
\hline 4,640 & 145.37 & $03-08-91$ & - & - & - & - & - & \\
\hline 4,500 & 25.25 & $03-07-91$ & - & - & - & - & - & \\
\hline 4,680 & - & - & $440 \mathrm{P}$ & $09-06-73$ & - & - & - & \\
\hline 4,485 & - & - & $300 \mathrm{~F}$ & $03-04-91$ & - & - & - & \\
\hline 4,500 & .02 & 03-07-91 & $20 \mathrm{P}$ & $04-16-73$ & - & - & - & \\
\hline 4,440 & -30.2 & $03-08-91$ & $30 \mathrm{~F}$ & $03-08-91$ & 11.5 & 465 & 03-08-91 & W,C,D \\
\hline 4,440 & - & - & - & - & - & - & - & \\
\hline 4,480 & -4.8 & $03-05-87$ & - & - & - & - & - & $w$ \\
\hline 4,480 & 2.66 & $03-11-91$ & - & - & - & - & - & \\
\hline 4,510 & 22.82 & 03-06-91 & - & - & - & - & - & \\
\hline 4,520 & 33.79 & $03-07-91$ & $2,754 \mathrm{P}$ & $08-20-90$ & - & - & - & \\
\hline 4,550 & 5.25 & $03-08-91$ & - & - & - & - & - & $w$ \\
\hline 4,580 & - & - & - & - & - & - & - & \\
\hline 4,680 & - & - & $1,400 P$ & $02-10-78$ & - & - & - & \\
\hline 4,750 & 38.26 & $03-07-91$ & - & - & - & - & - & \\
\hline 4,600 & 126.78 & $03-08-91$ & $1,690 \mathrm{P}$ & $07-28-77$ & - & - & - & w \\
\hline 4,570 & 79.90 & 03-06-91 & - & - & - & - & - & \\
\hline 4,610 & - & - & - & - & - & - & - & \\
\hline 4,490 & 13.44 & 03-11-91 & - & - & - & - & - & \\
\hline 4,740 & 37.82 & 04-03-91 & - & - & - & - & - & \\
\hline 4,540 & 59.11 & $04-03-91$ & - & - & - & - & - & \\
\hline 4,510 & 25.37 & 03-08-91 & - & - & - & - & - & $w$ \\
\hline 4,480 & -3.6 & 03-14-91 & $.49 \mathrm{~F}$ & $=03-14-91$ & 10.5 & 660 & 03-14-91 & \\
\hline
\end{tabular}


Table 1.-Records of

\begin{tabular}{|c|c|c|c|c|c|c|c|c|}
\hline \multirow[b]{2}{*}{ Location } & \multirow[b]{2}{*}{ Owner or user } & \multirow[b]{2}{*}{$\begin{array}{l}\text { Year } \\
\text { drilled }\end{array}$} & \multirow{2}{*}{$\begin{array}{l}\text { Use } \\
\text { of } \\
\text { water }\end{array}$} & \multirow{2}{*}{$\begin{array}{l}\text { Depth } \\
\text { of well } \\
\text { (feet) }\end{array}$} & \multicolumn{4}{|c|}{ Casing } \\
\hline & & & & & $\begin{array}{l}\text { Diameter } \\
\text { (inches) }\end{array}$ & $\begin{array}{l}\text { Depth } \\
\text { (feet) }\end{array}$ & & $\begin{array}{l}\text { Finish } \\
\text { (feet) }\end{array}$ \\
\hline$(A-12-1) 3 d b d-1$ & Hyde Park town & 1970 & $P$ & 245 & 12 & 275 & $\mathrm{P}$ & $95-250$ \\
\hline$(A-12-1) 4 a b b-1$ & Hinds, James & 1939 & $s$ & 182 & 3 & 152 & 0 & \\
\hline$(A-12-1) 5 d b b-1$ & Chambers, George & 1929 & $s$ & 208 & 2 & - & - & \\
\hline$(A-12-1) 6 b c c-1$ & Reese, C.L. & 1959 & $s$ & 585 & 2 & - & $s$ & $\begin{array}{l}504-574 \\
574-585\end{array}$ \\
\hline$(A-12-1) 7$ add-1 & Seamons, C. & 1961 & $s$ & 182 & 2 & 182 & $\mathrm{P}$ & $172-182$ \\
\hline$(A-12-1) 7 b b c-1$ & Cowley, George & 1918 & - & 441 & 2 & - & - & \\
\hline$(A-12-1) 7 b c b-1$ & Maughan, J.C. & 1954 & H & 470 & 2 & 470 & $P$ & $459-470$ \\
\hline$(A-12-1) 8 a c b-1$ & Lee, R.E. & 1895 & s & 60 & 2 & - & - & \\
\hline$(A-12-1) 9 b b d-2$ & Jensen Brothers & 1971 & 1 & 280 & 4 & 220 & 0 & \\
\hline$(A-12-1) 10 c b b-1$ & Hancey, H.E. & 1915 & $s$ & 106 & 2 & - & - & \\
\hline$(A-12-1) 11 b a a-1$ & Hyde Park town & 1968 & $P$ & 622 & 16 & - & $\mathrm{P}$ & - \\
\hline$(A-12-1) 12 c b c-1$ & Nelson, Lyle & 1986 & $\mathrm{H}$ & 480 & 4 & 474 & $P$ & 414-474 \\
\hline$(A-12-1) 16 c a c-1$ & Benson Irrigation Co. & 1929 & 1 & 44 & 4 & - & 0 & \\
\hline$(A-12-1) 17$ daa -1 & Benson Irrigation Co. & 1942 & 1 & 144 & 4 & - & $\mathrm{P}$ & $118-(?)$ \\
\hline$(A-12-1) 17$ daa-2 & U.S. Geological Survey & 1990 & $u$ & 19.6 & 2 & 19.6 & $\mathrm{~s}$ & $17-19$ \\
\hline$(A-12-1) 18 d d c-1$ & Hoffman, Alton & 1962 & 1 & 337 & 4 & 337 & 0 & \\
\hline$(A-12-1) 19 b d c-1$ & Spiers, Bernard \& Steven & 1983 & s & 134 & 10,6 & 134 & $\mathbf{P}$ & $114-134$ \\
\hline$(A-12-1) 20$ daa-2 & Quale, J.W. & 1944 & $s$ & 55 & 2 & - & $\mathbf{P}$ & 45-(?) \\
\hline$(A-12-1) 21 a d a-1$ & Rich, Russell E. & 1940 & - & 74 & 2 & - & - & \\
\hline$(A-12-1) 21$ caa-2 & Jensen, H. \& R. & 1972 & s & 140 & 3 & 140 & 0 & \\
\hline$(A-12-1) 21 d a a-1$ & North Logan city & 1973 & $P$ & 444 & 16 & 444 & $\mathrm{P}$ & $\begin{array}{l}284-325 \\
346-430\end{array}$ \\
\hline$(A-12-1) 23 a d d-1$ & Yeates, Brent & 1990 & $H$ & 350 & 6,5 & 350 & $\mathbf{P}$ & $316-350$ \\
\hline$(A-12-1) 24 a d a-1$ & Neilson, $M$. & - & $H$ & - & - & - & - & \\
\hline$(A-12-1) 24$ add -1 & North Logan city & 1990 & $P$ & 498 & 16,12 & 498 & $\mathbf{P}$ & $\begin{array}{l}152-209 \\
248-343 \\
363-498\end{array}$ \\
\hline$(A-12-1) 27 c a b-1$ & Logan city & 1963 & $P$ & 800 & 20,16 & 800 & $\mathrm{P}$ & $\begin{array}{l}350-370 \\
397-417 \\
473-493 \\
540-560 \\
600-620 \\
650-790\end{array}$ \\
\hline$(A-12-1) 27 d c d-1$ & Logan city & 1961 & $P$ & 470 & $20,16,12$ & 467 & $P$ & $\begin{array}{l}315-325 \\
335-365 \\
375-385 \\
395-440\end{array}$ \\
\hline$(A-12-1) 28 b d d-1$ & Jones, John C. & 1986 & 1 & 162 & 12,8 & 162 & $P$ & $143-159$ \\
\hline$(A-12-1) 29 c a b-1$ & Gossner, Edward & 1900 & $u$ & 43 & 2 & - & - & \\
\hline$(A-12-1) 29 d a d-1$ & Jones, John C. & 1981 & 1 & 164 & 16,10 & 164 & $P$ & $128-159$ \\
\hline$(A-12-1) 30 a d c-1$ & Lundberg, Heber & 1930 & - & 41 & 2 & - & - & \\
\hline (A-12-1)31bdc-1 & Gumand, T.L. & 1934 & - & 198 & 2 & - & - & \\
\hline$(A-12-1) 31$ dab-2 & Nish, R.T. & 1976 & 1 & - & 4 & - & - & \\
\hline$(A-12-1) 32 d b a-1$ & Hunsaker, T.E. & 1950 & $H$ & 119 & 2 & - & $P$ & $109-119$ \\
\hline
\end{tabular}




\begin{tabular}{|c|c|c|c|c|c|c|c|c|}
\hline \multirow{4}{*}{$\begin{array}{c}\text { Altitude } \\
\text { of land } \\
\text { surface } \\
\text { (feet) }\end{array}$} & \multicolumn{2}{|c|}{ Water level } & & & \multirow{2}{*}{\multicolumn{3}{|c|}{ Water-quality parameters }} & \multirow{4}{*}{$\begin{array}{c}\text { Other } \\
\text { data } \\
\text { available }\end{array}$} \\
\hline & \multirow{3}{*}{$\begin{array}{c}\text { Above (-) } \\
\text { or below } \\
\text { land surface } \\
\text { (feet) }\end{array}$} & \multirow[b]{3}{*}{ Date } & \multirow{2}{*}{\multicolumn{2}{|c|}{ Yiold }} & & & & \\
\hline & & & & & \multicolumn{3}{|c|}{ Specific } & \\
\hline & & & $\begin{array}{c}\text { Rate } \\
\text { (gal/min) }\end{array}$ & Date & $\begin{array}{c}\text { Temperature } \\
\left({ }^{\circ} \mathrm{C}\right)\end{array}$ & $\begin{array}{c}\text { conductance } \\
(\mu S / \mathrm{cm})\end{array}$ & Date & \\
\hline 4,520 & 39.06 & $04-02-91$ & $1,350 P$ & $11-10-70$ & - & - & - & \\
\hline 4,485 & 3.12 & $04-02-91$ & - & - & - & - & - & \\
\hline 4,435 & -19.8 & $03-28-91$ & $8.6 \mathrm{~F}$ & $03-28-91$ & 13.0 & 465 & $03-28-91$ & \\
\hline 4,435 & -21.3 & $03-19-91$ & $5.4 \mathrm{~F}$ & $03-19-91$ & 15.5 & 1,000 & $03-19-91$ & \\
\hline 4,435 & -8.5 & $04-02-91$ & $5.4 \mathrm{~F}$ & $04-02-91$ & 13.0 & 460 & $04-02-91$ & \\
\hline 4,433 & - & - & - & - & - & - & - & \\
\hline 4,430 & -34.9 & $03-19-91$ & $8.6 \mathrm{~F}$ & $03-19-91$ & 15.5 & 1,460 & $03-19-91$ & \\
\hline 4,440 & -8.5 & $04-02-91$ & 4.3 F & $04-02-91$ & 12.5 & 530 & $04-02-91$ & \\
\hline 4,440 & -8.1 & $03-29-91$ & $.9 \mathrm{~F}$ & $03-29-91$ & 11.0 & 750 & $03-29-91$ & \\
\hline 4,460 & -21.1 & $04-03-91$ & $7.5 \mathrm{~F}$ & 04-03-91 & 13.5 & 540 & $04-03-91$ & \\
\hline 4,760 & 212.09 & $04-02-91$ & - & - & - & - & - & \\
\hline 4,900 & 404.80 & $04-03-91$ & - & - & - & - & - & \\
\hline 4,455 & -10.8 & $04-02-91$ & $12 \mathrm{~F}$ & $04-02-91$ & 14.5 & 700 & $04-02-91$ & \\
\hline 4,450 & -13.5 & $03-09-91$ & $3.3 \mathrm{~F}$ & $03-09-91$ & 20.0 & 490 & 03-09-91 & W,C,D \\
\hline 4.450 & 1.09 & $03-18-91$ & - & - & - & - & - & \\
\hline 4,428 & -6.4 & $03-26-91$ & - & - & 8.5 & - & $03-26-91$ & \\
\hline 4,428 & -16.2 & $03-26-91$ & $1.2 \mathrm{~F}$ & $03-26-91$ & 10.5 & 470 & $03-26-91$ & \\
\hline 4,455 & -7.4 & $03-21-91$ & $7.3 \mathrm{~F}$ & $03-21-91$ & 16.5 & 510 & $03-21-91$ & \\
\hline 4,490 & - & - & - & - & - & - & - & \\
\hline 4,550 & -7.2 & $04-02-91$ & - & - & 13.0 & - & $04-02-91$ & \\
\hline 4,495 & 54.00 & $04-02-91$ & - & - & - & - & - & $\mathbf{L}$ \\
\hline 4,780 & - & - & - & - & - & - & - & \\
\hline 5,060 & - & - & - & - & - & - & - & \\
\hline 5,060 & 64.57 & $04-02-91$ & $600 P$ & $11-29-90$ & 一 & - & - & w \\
\hline 4,550 & - & - & - & - & - & - & - & \\
\hline 4,625 & - & - & - & - & - & - & - & \\
\hline 4,485 & 2.80 & $03-06-91$ & - & - & - & - & - & \\
\hline 4,440 & -12.3 & $03-06-91$ & - & - & 16.5 & 500 & 03-06-91 & $w, C$ \\
\hline 4,475 & - & - & $500 \mathrm{~F}$ & $03-06-91$ & - & - & - & \\
\hline 4,428 & - & - & - & - & - & - & - & \\
\hline 4,432 & - & - & - & - & - & - & - & \\
\hline 4,430 & -26.8 & $03-07-91$ & $8.6 \mathrm{~F}$ & $03-01-90$ & 14.5 & 415 & 03-07-91 & $W, C$ \\
\hline & & & $6.7 \mathrm{~F}$ & $04-27-90$ & & & & \\
\hline 4,450 & -3.7 & $03-13-91$ & $1.37 \mathrm{~F}$ & $03-13-91$ & 12.0 & 470 & $03-13-91$ & \\
\hline
\end{tabular}


Table 1.-Records of

\begin{tabular}{|c|c|c|c|}
\hline Location & Owner or user & $\begin{array}{c}\text { Year } \\
\text { drilled }\end{array}$ & $\begin{array}{c}\text { Use } \\
\text { of } \\
\text { water }\end{array}$ \\
\hline$(A-12-1) 32 d b d-2$ & L.D.S. Church & 1970 & 1 \\
\hline (A-12-1)33bac-3 & Jorgensen, Ester & - & $u$ \\
\hline$(A-12-1) 33 b c a-1$ & Benson, K.E. & 1953 & s \\
\hline$(A-12-1) 33 b c c-3$ & Eliason Packing Co. & 1940 & $\mathbf{S}$ \\
\hline$(A-12-1) 34 c c a-1$ & Logan city & 1963 & $\mathbf{P}$ \\
\hline$(A-12-1) 34 c c c-1$ & Logan Island Irrigation Co. & 1934 & 1 \\
\hline (A-12-1)35bba-1 & Utah State University & 1983 & $T$ \\
\hline$(A-12-1) 35 b c c-1$ & Logan city & 1963 & $\mathbf{P}$ \\
\hline
\end{tabular}

(A-13-1)3bab-1

(A-13-1)3cab-1

(A-13-1)9abb-1

(A-13-1)10dba-1

(A-13-1)10dba-2

(A-13-1)11bbb-1

(A-13-1)16ccb-1

(A-13-1) 17abb-2

(A-13-1)20aca-1

(A-13-1)20acb-1

(A-13-1)20acb-2

(A-13-1)20acb-3

(A-13-1)20acc-2

(A-13-1)28aaa-1

(A-13-1)28cab-1

(A-13-1)28ccd-1

(A-13-1)28dab-1

(A-13-1)28dbb-1

(A-13-1)29adc-1

(A-13-1)31ccc-1

(A-13-1)32daa-1

(A-13-1)34bcb-1
Bair, F.A.

Mendonhall, O.J.

-

Eaves, Phil

Eaves, Phil

Mendonhall, O.J.

Miles, A.A.

Tout, Phil

Amalga town

Amalga town

Amalga town

Amalga town

Fisher, G.R.

Logan Northem Irrigation Co.

Smithfield city

Chambers, G.L.

Smithfield Irrigation Co.

Smithfield Irrigation Co.

Cannell, J.C.

Reese, A.C.

Weeks, David

Del Monte Corporation
R \& J Farms

(A-13-1)35ccc-

(A-14-1)2bbd-1

(A-14-1)2ccd-1

(A-14-1) 5cdb-1

(A-14-1)6ccc-1
Larsen, C.M.

Larsen, S.W.

Rupp, Milo A.

Karren, Fred
Depth

(feet)

\begin{tabular}{rcl}
\multicolumn{4}{c}{ Casing } \\
\hline $\begin{array}{c}\text { Diameter } \\
\text { (inches) }\end{array}$ & $\begin{array}{c}\text { Depth } \\
\text { (feet) }\end{array}$ & $\begin{array}{c}\text { Finish } \\
\text { (feet) }\end{array}$ \\
\hline 8 & 140 & 0 \\
2 & - & - \\
2 & - & - \\
- & - & - \\
20,16 & 975 & P $485-530$ \\
& &
\end{tabular}

119

434

978

$\begin{array}{rr}12 & 119 \\ 12,8 & 434 \\ 20 & -\end{array}$

P $380-435$

P $305-330$

410-450

510-525

$650-750$

830-880

925-945

1926

1930

-

1983

1951

1918

-

1974

1921

1947

1925

1962

1968

125

125

-

175

15

21

90

230

114

107

139

98

90

477

210

1967

1954

1961

1919

1918

1953

1953

N

P
$H$
$I$
U
S
I
N

$$
131
$$$$
235
$$$$
315
$$$$
106
$$$$
400
$$$$
131
$$$$
315
$$

1990

1953

1949

1965
200

160

105

7

20
$-$

-

- -

175 P 100-175

150

21 P 11-(?)

- -

-

114 P 86-108

1070

139 P $105-139$

$98 \quad P \quad 88-96$

- -

477 P $181-474$

$210 \quad P \quad 122-158$

190-210

1310

- P 105-(?)

$315 \quad P$ -

1060

$400 \quad 0$

- P 101-111

315 P $135-140$

206-216

262-267

282-284

313-315

6199 P 160-195

4156 P $146-150$

$4 \quad-\quad P \quad 82-92$

$48-0$

36 - - 


\begin{tabular}{|c|c|c|c|c|c|c|c|c|}
\hline \multirow{3}{*}{$\begin{array}{c}\text { Altitude } \\
\text { of land } \\
\text { surface } \\
\text { (feet) }\end{array}$} & \multicolumn{2}{|c|}{ Water lovel } & & & \multirow{2}{*}{\multicolumn{3}{|c|}{ Water-quality parameters }} & \multirow{3}{*}{$\begin{array}{c}\text { Other } \\
\text { data } \\
\text { available }\end{array}$} \\
\hline & $\begin{array}{l}\text { Above }(-) \\
\text { or below }\end{array}$ & & \multicolumn{2}{|c|}{ Yield } & & & & \\
\hline & $\begin{array}{c}\text { land surface } \\
\text { (feet) }\end{array}$ & Date & $\begin{array}{c}\text { Rate } \\
\text { (gal/min) }\end{array}$ & Date & $\begin{array}{c}\text { Temperature } \\
\left({ }^{\circ} \mathrm{C}\right)\end{array}$ & $\begin{array}{c}\text { conductance } \\
(\mu \mathrm{S} / \mathrm{cm})\end{array}$ & Date & \\
\hline 4,450 & - & - & - & - & - & - & - & \\
\hline 4,486 & 6.19 & $03-13-91$ & - & - & - & - & - & \\
\hline 4,480 & -5.8 & 03-13-91 & 4.76 & 3-13-91 & 13.0 & 410 & 03-13-91 & \\
\hline 4,470 & -4.7 & 03-13-91 & - & - & 11.5 & 410 & 03-13-91 & \\
\hline 4,550 & - & - & - & - & - & - & - & \\
\hline 4,530 & 44.09 & $03-08-91$ & - & - & - & - & - & w \\
\hline 4,785 & - & - & - & - & - & - & - & D \\
\hline 4,600 & - & - & - & - & - & - & - & $\mathrm{L}$ \\
\hline
\end{tabular}

$\begin{array}{cc}4,495 & -5.2 \\ 4,495 & -14.7 \\ 4,470 & - \\ 4,580 & 32.30 \\ 4,560 & 7.41 \\ 4,625 & 9.30 \\ 4,480 & 5.06 \\ 4,460 & -20.6 \\ 4,460 & - \\ 4,460 & - \\ 4,460 & - \\ 4,460 & - \\ 4,465 & -8.7 \\ 4,595 & 118.00 \\ & 115.32 \\ 4,530 & -\end{array}$

04-02-91

04-03-91

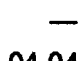

04-04-91

04-04-91

04-04-91

03-28-91

03-28-91

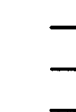

$-$

03-28-91

03-14-91

04-03-91

-

04-03-91

4,510

4,570

4,545

4,490

4,440

4,475

4,555

18.24

98.40

67.26

12.53

$-8.1$

$-1.1$

69.71

04-02-91

$\begin{array}{lr}4,620 & 138.10 \\ 4,490 & 23.40 \\ 4,490 & 27.47 \\ 4,505 & 2.62 \\ 4,505 & 5.85\end{array}$

04-02-91

03-28-91

03-28-91

03-28-91

09-23-86

$\begin{array}{ccccc}.25 \mathrm{~F} & 04-02-91 & 9.0 & 680 & 04-02-91 \\ - & - & 6.5 & 660 & 04-03-91 \\ - & - & - & - & - \\ - & - & - & - & - \\ - & - & - & - & - \\ - & - & - & - & - \\ - & - & - & - & - \\ 150 \mathrm{~F} & 03-28-91 & 13.0 & 1,390 & 03-28-91 \\ - & - & - & - & - \\ - & - & - & - & - \\ - & - & - & - & - \\ - & - & - & - & - \\ 12 \mathrm{~F} & 03-28-91 & 11.5 & 475 & 03-28-91 \\ 3,560 \mathrm{P} & 08-21-90 & - & - & - \\ - & - & - & - & - \\ - & - & - & - & -\end{array}$

$\begin{array}{ccr}\overline{1} & - & - \\ 3600 \text { P } & 08-03-90 & - \\ - & 08-19-87 & - \\ .3 \text { F } & 03-19-91 & - \\ 1.8 \mathrm{~F} & 03-28-91 & 12.0 \\ - & - & -\end{array}$

w

940 03-19-91

$600 \quad 03-28-91$

$\begin{array}{ccccc}285 P & 08-24-90 & - & - & - \\ - & - & - & - & - \\ - & - & - & - & - \\ - & - & - & - & - \\ - & - & - & - & -\end{array}$


Table 1.-Records of

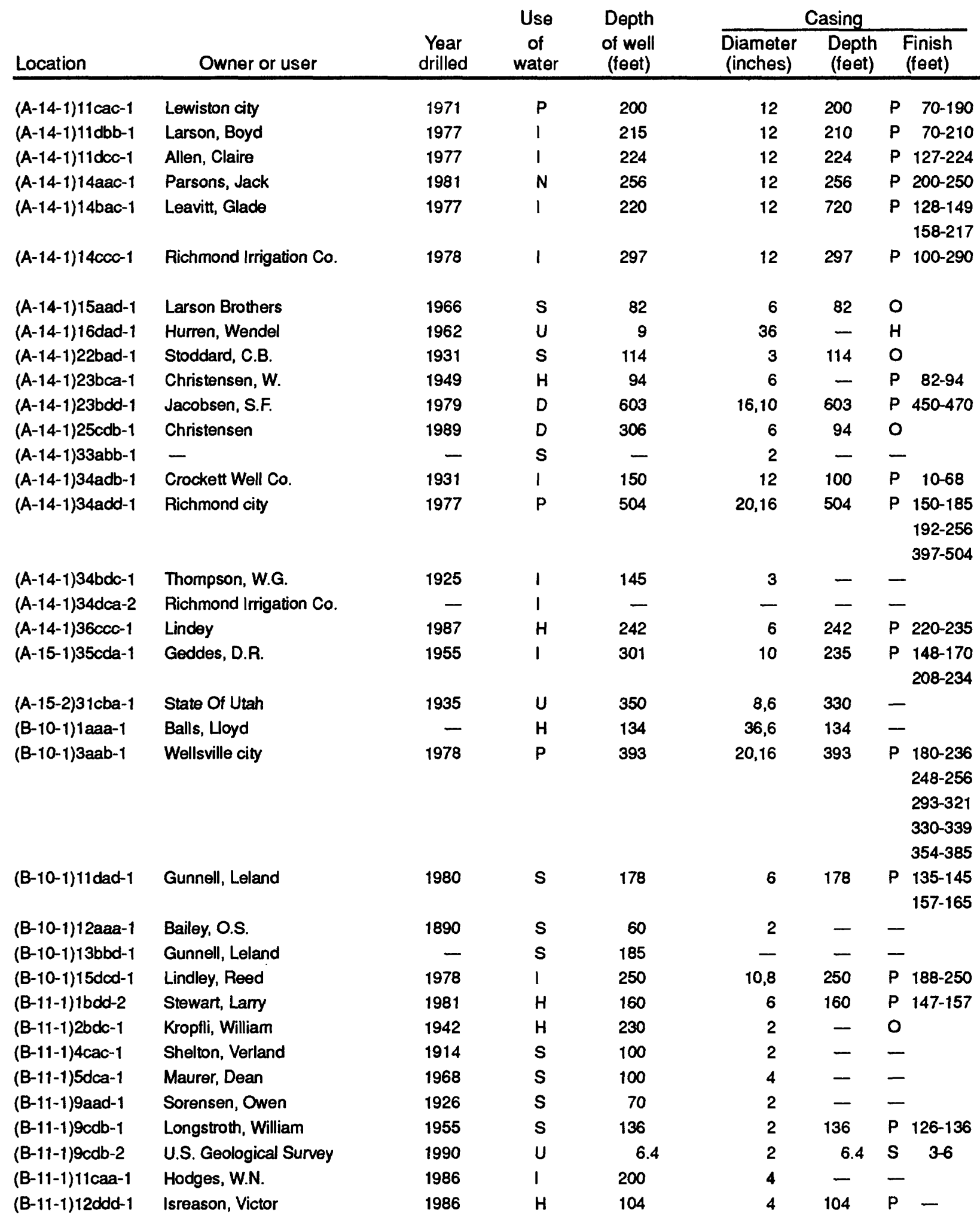




\begin{tabular}{|c|c|c|c|c|c|c|c|c|}
\hline \multirow{3}{*}{$\begin{array}{l}\text { Altitude } \\
\text { of land } \\
\text { surface } \\
\text { (feet) }\end{array}$} & \multicolumn{2}{|c|}{ Water level } & & \multirow{2}{*}{\multicolumn{3}{|c|}{ Water-quality parameters }} & \multirow{3}{*}{$\begin{array}{c}\text { Other } \\
\text { data } \\
\text { available }\end{array}$} \\
\hline & \multirow{2}{*}{$\begin{array}{c}\text { Above (-) } \\
\text { or below } \\
\text { land surface } \\
\text { (feet) }\end{array}$} & \multirow[b]{2}{*}{ Date } & & & & & & \\
\hline & & & $\begin{array}{c}\text { Yield } \\
\text { Rate } \\
\text { (gal/min) }\end{array}$ & Date & $\begin{array}{c}\text { Temperature } \\
\left({ }^{\circ} \mathrm{C}\right)\end{array}$ & $\begin{array}{c}\text { Specific } \\
\text { conductance } \\
(\mu \mathrm{S} / \mathrm{cm})\end{array}$ & Date & \\
\hline 4,525 & 55.08 & $04-04-91$ & - & - & - & - & - & \\
\hline 4,530 & 60.34 & $03-07-89$ & - & - & - & - & - & W \\
\hline 4,560 & 91.84 & $03-13-91$ & $490 P$ & $08-01-90$ & - & - & - & D \\
\hline 4,700 & - & - & $450 P$ & $08-20-90$ & - & - & - & \\
\hline \multirow[t]{2}{*}{4,555} & 89.84 & 03-13-91 & $1,850 P$ & $01-10-78$ & - & - & - & \\
\hline & & & $406 P$ & $08-20-90$ & & & & \\
\hline \multirow[t]{2}{*}{4,530} & 73.48 & $03-08-91$ & $1,820 P$ & $09-13-78$ & - & - & - & W \\
\hline & & & $1,380 P$ & $08-01-90$ & & & & \\
\hline 4,515 & 50.33 & $03-13-91$ & - & - & - & - & - & \\
\hline 4,500 & 7.90 & $04-02-91$ & - & - & - & - & - & \\
\hline 4,466 & -0.7 & $03-08-91$ & $3.5 \mathrm{~F}$ & $07-24-90$ & 10.0 & 485 & $07-24-90$ & W,C,D \\
\hline 4,555 & 83.37 & $04-02-91$ & - & - & - & - & - & \\
\hline 4,600 & 56.78 & $04-02-91$ & - & - & - & - & - & $L$ \\
\hline 4,960 & 15.23 & $04-02-91$ & - & - & - & - & - & \\
\hline 4,440 & -5.6 & $04-03-91$ & $2.0 \mathrm{~F}$ & 04-03-91 & - & - & - & \\
\hline 4,540 & 20.20 & $03-08-91$ & $426 P$ & $08-19-76$ & - & - & - & $w$ \\
\hline 4,580 & 95.00 & $04-04-91$ & $751 P$ & $11-30-77$ & - & - & - & $L$ \\
\hline 4,490 & -13.7 & $04-02-91$ & $12.9 \mathrm{~F}$ & $04-02-91$ & 10.5 & 660 & $04-02-91$ & \\
\hline 4,520 & - & - & - & - & - & - & - & \\
\hline 5,020 & 182.60 & $04-03-91$ & - & - & 10.5 & 660 & $04-02-91$ & \\
\hline 4,475 & 6.32 & $03-28-91$ & - & - & - & - & - & \\
\hline 4,620 & -1.1 & $04-01-91$ & $.42 \mathrm{~F}$ & $=04-01-91$ & 13.5 & 300 & $04-01-91$ & L \\
\hline 4,580 & 97.19 & $03-12-91$ & - & - & - & - & - & \\
\hline 4,540 & - & - & $860 P$ & $04-17-78$ & - & - & - & $L$ \\
\hline 4,680 & 20.75 & $03-06-91$ & - & - & - & - & - & $w$ \\
\hline 4,640 & -8.3 & $03-12-91$ & - & - & 11.0 & 640 & $03-12-91$ & \\
\hline 4,800 & - & - & - & - & - & - & - & \\
\hline 4,840 & - & - & - & - & - & - & - & \\
\hline 4,430 & -46.2 & 03-19-91 & $50 \mathrm{~F}$ & $01-29-81$ & 10.0 & 620 & 03-19-91 & \\
\hline 4,415 & -24.8 & $03-20-91$ & - & - & - & - & - & \\
\hline 4,415 & -6.4 & $03-18-91$ & $1.71 \mathrm{~F}$ & $03-18-91$ & 10.5 & 790 & $03-18-91$ & \\
\hline 4,475 & 7.04 & $03-18-91$ & - & - & - & - & - & \\
\hline 4,415 & -3.0 & 03-18-91 & $.73 F$ & 03-18-91 & 10.0 & 800 & $03-18-91$ & \\
\hline 4,420 & -5.5 & $03-06-91$ & $.6 \mathrm{~F}$ & $03-06-91$ & 9.5 & 970 & $03-06-91$ & $W, C, D$ \\
\hline 4,420 & 1.31 & $03-06-91$ & - & - & - & - & - & \\
\hline 4,425 & -12.6 & $03-19-91$ & $15 \mathrm{~F}$ & $03-19-91$ & 12.0 & 620 & 03-19-91 & \\
\hline 4,435 & -31.0 & $03-19-91$ & $185 \mathrm{~F}$ & $06-10-86$ & 10.5 & 490 & 03-19-91 & \\
\hline
\end{tabular}


Table 1.—Records of

\begin{tabular}{|c|c|c|c|c|c|c|c|c|}
\hline \multirow[b]{2}{*}{ Location } & \multirow[b]{2}{*}{ Owner or user } & \multirow[b]{2}{*}{$\begin{array}{l}\text { Year } \\
\text { drilled }\end{array}$} & \multirow{2}{*}{$\begin{array}{l}\text { Use } \\
\text { of } \\
\text { water }\end{array}$} & \multirow{2}{*}{$\begin{array}{l}\text { Depth } \\
\text { of well } \\
\text { (feet) }\end{array}$} & \multicolumn{4}{|c|}{ Casing } \\
\hline & & & & & $\begin{array}{l}\text { Diameter } \\
\text { (inches) }\end{array}$ & $\begin{array}{c}\text { Depth } \\
\text { (feet) }\end{array}$ & & $\begin{array}{l}\text { Finish } \\
\text { (feet) }\end{array}$ \\
\hline (B-11-1)13acd-1 & Jensen, C.A. & 1949 & H & 95 & 2 & - & $\mathbf{P}$ & 85-95 \\
\hline (B-11-1) 14aaa-3 & L.D.S. Young Ward & 1951 & $\mathrm{~N}$ & 203 & 2 & - & $\mathbf{P}$ & $193-203$ \\
\hline (B-11-1) 14cab-1 & Lewis, T., \& Jensen Brothers & 1969 & 1 & 283 & 12,8 & 283 & $\mathbf{P}$ & $239-264$ \\
\hline (B-11-1)14cdb-1 & Olsen, Evan & 1983 & 1 & 310 & 12,8 & 310 & $\mathrm{P}$ & $\begin{array}{l}241-263 \\
266-271 \\
281-310\end{array}$ \\
\hline$(B-11-1) 14 c d c-1$ & Stuart, Charles & 1917 & 1 & 220 & 3 & - & - & \\
\hline (B-11-1)14codd-3 & Glen, Mary & 1985 & 1 & 296 & 12,8 & 296 & $\mathbf{P}$ & $\begin{array}{l}174-189 \\
230-248 \\
254-265 \\
277-290\end{array}$ \\
\hline (B-11-1)15cbb-1 & Hardman, E.B. & 1896 & $s$ & 75 & 2 & - & - & \\
\hline (B-11-1) 15dba-1 & Anderson, Willis & 1933 & s & 160 & 2 & - & $\mathbf{P}$ & - \\
\hline (B-11-1)16aca-1 & Sorensen, Claud & 1937 & s & 82 & 2 & 22 & 0 & \\
\hline (B-11-1)16add-1 & Sorensen, Henry & 1919 & s & 80 & 2 & - & - & \\
\hline$(B-11-1) 16 c c c-1$ & Moon, Marvin & - & H & 250 & - & - & - & \\
\hline (B-11-1)17add-1 & Mendon city & 1978 & $P$ & 148 & 10 & 148 & $\mathbf{P}$ & $120-148$ \\
\hline$(B-11-1) 21 a b d-1$ & Leishman, Donald $P$. & 1977 & s & 135 & 3 & 135 & 0 & \\
\hline$(B-11-1) 22 c b d-1$ & Haslam, Legrand & 1935 & $u$ & 132 & 2 & 100 & 0 & \\
\hline (B-11-1)22cdb-1 & Murray, Henry & 1962 & $s$ & 75 & 2 & 75 & $\mathbf{P}$ & $65-75$ \\
\hline$(B-11-1) 23 b b c-1$ & Hill, Ray & - & 1 & 270 & 10 & 269 & $P$ & $\begin{array}{l}186-191 \\
235-268\end{array}$ \\
\hline$(B-11-1) 23 d c b-1$ & Anderson, Eldon & 1918 & s & 166 & 2 & - & - & \\
\hline (B-11-1)24acd-1 & Anderson, Hyrum & 1915 & $s$ & 120 & 2 & - & - & \\
\hline$(B-11-1) 25 \mathrm{cda}-2$ & Utah State University & 1981 & $H$ & 212 & 8 & 212 & $\mathbf{P}$ & $187-207$ \\
\hline$(B-11-1) 26 a b d-1$ & Bankhead, Heber & 1921 & s & 55 & 2 & - & 一 & \\
\hline (B-11-1)27bbb-1 & - & - & $u$ & 29 & 30 & - & - & \\
\hline$(B-11-1) 27 c d c-1$ & Darley, R.M. & 1972 & H & 194 & 6 & 188 & 0 & \\
\hline$(B-11-1) 33$ daa-1 & Maughn, Curtis & 1986 & H & 297 & 6 & 297 & $\mathbf{P}$ & $260-295$ \\
\hline (B-11-1)34bac-1 & Hamilton, Dixon & 1987 & H & 173 & 6 & 173 & $P$ & $160-167$ \\
\hline (B-11-1)35caa-1 & Leishman, James A. & 1923 & - & 65 & 2 & - & - & \\
\hline (B-11-1)35caa-2 & Leishman, James A. & 1923 & s & 120 & - & - & - & \\
\hline$(B-11-1) 35 \propto c a-1$ & Brenchley, John & 1923 & s & 65 & 2 & 65 & 0 & \\
\hline$(B-12-1) 1 c c c-2$ & Ballard, M.J. & 1956 & $\mathrm{H}$ & 590 & 2 & - & $P$ & $584-(?)$ \\
\hline (B-12-1)2bab-1 & Falslev, Chad & 1987 & $H$ & 555 & 4,2 & 555 & s & $545-555$ \\
\hline (B-12-1)3ccc-1 & Watterson, J.L. & - & H & - & 2 & - & - & \\
\hline$(B-12-1) 8 c d b-2$ & Rasmussen, Reuben & 1889 & s & 210 & 2 & 30 & 0 & \\
\hline (B-12-1)9ddd-1 & Benson Recreation Area & 1969 & $u$ & 576 & 2 & - & 0 & \\
\hline (B-12-1)10dcd-2 & Nuttall, J.L. & 1954 & $H$ & 533 & 2 & - & $P$ & $522-(?)$ \\
\hline$(B-12-1) 11 b b c-4$ & Johnson, W.D. & 1990 & s & 606 & 4,2 & 502 & $s$ & $326-336$ \\
\hline (B-12-1)11dda-1 & Snow, F.B. & 1962 & $\mathrm{H}$ & 545 & 2 & 545 & $P$ & $535-545$ \\
\hline (B-12-1)14aaa-1 & Riggs, Bert & 1929 & s & 304 & 2 & - & - & \\
\hline (B-12-1)15adc-1 & Ricks, Charles Ethan & 1941 & 1 & 418 & 2 & 418 & 0 & \\
\hline
\end{tabular}




\begin{tabular}{|c|c|c|c|c|c|c|c|c|}
\hline \multirow{3}{*}{$\begin{array}{l}\text { Altitude } \\
\text { of land } \\
\text { surface } \\
\text { (feet) }\end{array}$} & \multicolumn{2}{|c|}{ Water level } & & \multirow{2}{*}{\multicolumn{3}{|c|}{ Water-quality parameters }} & \multirow{3}{*}{$\begin{array}{c}\text { Other } \\
\text { data } \\
\text { available }\end{array}$} \\
\hline & \multirow{2}{*}{$\begin{array}{c}\text { Above (-) } \\
\text { or below } \\
\text { land surface } \\
\text { (feet) }\end{array}$} & \multirow[b]{2}{*}{ Date } & & & & & & \\
\hline & & & $\frac{\text { Yield }}{\text { Rate }}$ & $\overline{\text { Date }}$ & $\begin{array}{c}\text { Temperature } \\
\left({ }^{\circ} \mathrm{C}\right)\end{array}$ & $\begin{array}{c}\text { Specific } \\
\text { conductance } \\
(\mu \mathrm{S} / \mathrm{cm})\end{array}$ & Date & \\
\hline 4,435 & -23.2 & $03-19-91$ & $100 \mathrm{~F}$ & $03-19-91$ & 11.5 & 540 & $03-19-91$ & \\
\hline 4,430 & -25.4 & $03-08-82$ & - & - & - & - & - & $w$ \\
\hline 4,435 & - & - & $42.5 \mathrm{~F}$ & 03-19-91 & - & - & - & \\
\hline 4,440 & - & - & - & - & - & - & - & $L$ \\
\hline 4,445 & -23.0 & 03-19-91 & - & 一 & - & - & - & \\
\hline 4,440 & - & - & - & - & - & - & - & \\
\hline 4,420 & -5.7 & 03-19-91 & $3.5 \mathrm{~F}$ & 03-19-91 & 10.0 & 680 & 03-19-91 & \\
\hline 4,420 & -19.1 & $03-19-91$ & $6.4 \mathrm{~F}$ & $03-19-91$ & 11.0 & 560 & $03-19-91$ & \\
\hline 4,420 & -7.5 & $03-18-91$ & $.73 F$ & $03-18-91$ & 10.0 & 610 & $03-18-91$ & \\
\hline 4,420 & -6.8 & $03-18-91$ & $.3 \mathrm{~F}$ & $03-18-91$ & 10.0 & 680 & 03-18-91 & \\
\hline 4,530 & 76.10 & $03-18-91$ & - & - & - & - & - & \\
\hline 4,500 & - & - & $824 P$ & $05-00-78$ & - & - & - & \\
\hline 4,440 & -7.0 & $03-12-91$ & $1.15 \mathrm{P}$ & $03-12-91$ & 10.5 & 590 & $03-12-91$ & \\
\hline 4,435 & -5.7 & $03-20-91$ & - & - & - & - & - & \\
\hline 4,440 & -0.67 & $03-12-91$ & - & - & - & - & - & \\
\hline 4,430 & - & - & - & - & - & - & - & \\
\hline 4,441 & -25.8 & $03-11-91$ & - & - & 11.0 & 660 & $03-11-91$ & \\
\hline 4,455 & -16.6 & $03-11-91$ & - & - & - & - & - & \\
\hline 4,485 & 11.76 & $03-06-91$ & - & - & - & - & - & \\
\hline 4,440 & -11.4 & $03-11-91$ & $.5 \mathrm{~F}$ & $03-11-91$ & 8.5 & 1,000 & $03-11-91$ & \\
\hline 4,485 & - & - & - & - & - & - & - & \\
\hline 4,600 & 56.02 & $03-12-91$ & - & - & - & - & - & \\
\hline 4,800 & 285.35 & $03-12-91$ & - & - & - & - & - & \\
\hline 4,580 & 78.31 & $03-12-91$ & - & - & - & - & - & \\
\hline $4,476.67$ & - & - & $.4 \mathrm{~F}$ & $03-01-90$ & - & - & - & \\
\hline & - & - & $.3 F$ & $06-28-90$ & - & - & - & \\
\hline 4,470 & 0 & $03-10-80$ & - & - & - & - & - & $w$ \\
\hline 4,475 & -3.6 & $03-06-91$ & - & - & 10.0 & 740 & $03-06-91$ & $W, C$ \\
\hline 4,435 & -34.0 & $03-20-91$ & - & - & 17.0 & 1,600 & $03-20-91$ & \\
\hline 4,422 & -51.7 & $03-20-91$ & $9.2 \mathrm{~F}$ & $03-20-91$ & 17.5 & 1,790 & 03-20-91 & \\
\hline 4,410 & -11.4 & $03-21-91$ & $8.6 \mathrm{~F}$ & 03-21-91 & 16.0 & 840 & $03-21-91$ & \\
\hline 4,430 & -5.5 & $03-09-91$ & $1.2 \mathrm{~F}$ & 03-09-91 & 12.0 & 730 & 03-09-91 & W,C,D \\
\hline 4,409 & -3.0 & $03-20-91$ & - & - & 18.5 & 900 & $03-20-91$ & \\
\hline 4,420 & -12.3 & $03-21-91$ & $6.0 \mathrm{~F}$ & $03-21-91$ & 23.5 & 820 & $03-21-91$ & \\
\hline 4,422 & - & - & $509 \mathrm{~F}$ & $09-06-90$ & - & - & - & \\
\hline 4,425 & -2.6 & $03-21-91$ & $6.7 \mathrm{~F}$ & $03-21-91$ & 17.0 & 1,500 & $03-21-91$ & \\
\hline 4,425 & -10.5 & $03-21-91$ & $20 \mathrm{~F}$ & $03-21-91$ & 14.5 & 475 & $03-21-91$ & \\
\hline 4,415 & -10.8 & 03-07-91 & $1.6 \mathrm{~F}$ & 03-17-91 & 17.0 & 860 & $03-07-91$ & W,C,D \\
\hline
\end{tabular}


Table 1.-Records of

\begin{tabular}{|c|c|c|c|c|c|c|c|c|}
\hline \multirow[b]{2}{*}{ Location } & \multirow[b]{2}{*}{ Owner or user } & \multirow[b]{2}{*}{$\begin{array}{l}\text { Year } \\
\text { drilled }\end{array}$} & \multirow{2}{*}{$\begin{array}{l}\text { Use } \\
\text { of } \\
\text { water }\end{array}$} & \multirow{2}{*}{$\begin{array}{l}\text { Depth } \\
\text { of well } \\
\text { (feet) }\end{array}$} & \multicolumn{4}{|c|}{ Casing } \\
\hline & & & & & $\begin{array}{l}\text { Diameter } \\
\text { (inches) }\end{array}$ & $\begin{array}{l}\text { Depth } \\
\text { (feet) }\end{array}$ & & $\begin{array}{l}\text { inish } \\
\text { (feet) }\end{array}$ \\
\hline (B-12-1) 15adc-2 & Ricks, Charles Ethan & - & 1 & 297 & 2 & 297 & $T$ & - \\
\hline (B-12-1)15adc-3 & U.S. Geological Survey & 1990 & u & 9.4 & 2 & 9.4 & s & $6-9$ \\
\hline$(B-12-1) 19 c c a-1$ & Hanson, Craig & 1983 & $P$ & 340 & 10 & 340 & $P 2$ & $200-340$ \\
\hline$(B-12-1) 19 d d d-1$ & Rosen, Vickie \& Cole, Brad & 1988 & H & 177 & 6 & 177 & $\mathbf{P}$ & $140-177$ \\
\hline (B-12-1) 19ddd-2 & Rosen, Vickie \& Cole, Brad & - & $H$ & 40 & 6 & - & - & \\
\hline (B-12-1)23dbd-1 & Benson, F.A. & 1905 & $\mathrm{~s}$ & 93 & 2 & - & - & \\
\hline$(B-12-1) 24 d a a-1$ & Waterson, J.L. & 1961 & 1 & 320 & 6 & 317 & $P 2$ & 295-315 \\
\hline$(B-12-1) 24 d a b-1$ & Hebaus, Joseph & 1919 & H & - & 2 & - & - & \\
\hline$(B-12-1) 26 a d a-1$ & Utah Power \& Light & - & s & 450 & 2 & - & - & \\
\hline$(B-12-1) 26 c c a-1$ & Schvaneveldt & 1944 & $\mathrm{~s}$ & 248 & 2 & 248 & 0 & \\
\hline$(B-12-1) 30 a b b-1$ & Hanson, Craig & 1983 & $\mathbf{P}$ & 240 & 8 & 240 & $P$ & $130-240$ \\
\hline$(B-12-1) 32 a b c-1$ & Johnson, Milton & - & $u$ & 460 & 3 & - & - & \\
\hline$(B-13-1) 10 b b a-1$ & Erickson, Lynn & 1964 & $u$ & 258 & 12 & 258 & $P$ & $50-250$ \\
\hline$(B-13-1) 16 c b d-1$ & Larsen, J.G. & 1971 & $H$ & 210 & 8 & 206 & $P$ & $50-200$ \\
\hline (B-13-1)17dad-2 & Larsen, O.G. & 1961 & s & 138 & 5 & 138 & $\mathbf{P}$ & $110-118$ \\
\hline$(B-13-1) 27 c b b-1$ & Anderson, Lynn & - & s & 40 & 2 & - & - & \\
\hline$(B-13-1) 27 c d d-1$ & Seamons, N.B. & 1961 & $H$ & 930 & 2 & 918 & $P$ & $908-918$ \\
\hline$(B-13-1) 28 a b b-1$ & Ringer, Wayne B. & 1970 & 1 & 130 & 8 & 130 & $P$ & $30-120$ \\
\hline$(B-13-1) 30 a c c-1$ & Ballard, E.R. & 1907 & $u$ & 90 & 2 & 90 & 0 & \\
\hline \multirow[t]{5}{*}{$(B-13-1) 30$ caa-1 } & Union Pacific Railroad & 1957 & $\mathbf{P}$ & 327 & 10 & 327 & $P$ & $70-78$ \\
\hline & & & & & & & & $145-157$ \\
\hline & & & & & & & & $196-200$ \\
\hline & & & & & & & & $251-254$ \\
\hline & & & & & & & & $301-315$ \\
\hline$(B-13-1) 30 \mathrm{cab}-1$ & Walton Feed Store & - & $\mathrm{N}$ & 176 & 6 & - & - & \\
\hline (B-13-1)30cba-2 & Walton Feed Store & - & H & 40 & 2 & - & - & \\
\hline$(B-13-1) 36 c c a-1$ & Thain, Paul & 1964 & H & 723 & 2 & 723 & 0 & \\
\hline$(B-14-1) 3 c d d-1$ & Buxton, Verl & - & $u$ & 271 & 2 & - & - & \\
\hline (B-14-1)5ddd-1 & Troseth, Soine & - & $H$ & 26 & 48 & - & - & \\
\hline \multirow[t]{3}{*}{ (B-14-1)17add-1 } & Town of Cornish & 1967 & $P$ & 92 & 8 & 92 & $\mathbf{P}$ & $51-56$ \\
\hline & & & & & & & & $58-68$ \\
\hline & & & & & & & & $69-85$ \\
\hline$(B-14-1) 28 c c a-1$ & Grancheff, Dave & 1979 & 1 & 215 & 10 & 116 & $P$ & $100-115$ \\
\hline$(B-14-1) 29 b b c-1$ & Ravsten, B.J. & 1900 & s & 24 & 30 & - & $w$ & \\
\hline (B-14-1)31aаa-1 & Buroau of Reclamation & 1955 & $u$ & 23 & 3 & 23 & - & \\
\hline (B-14-2)26ddb-1 & Raveston, J.N. & 1970 & 1 & 100 & 12 & 100 & $\mathbf{P}$ & $78-90$ \\
\hline (B-14-2)27ddd-1 & Jardine, Sterling & 1964 & $u$ & 70 & 4 & - & - & \\
\hline (B-14-2)35bca-1 & Bureau of Reclamation & 1954 & $u$ & 8 & 3 & - & $P$ & - \\
\hline \multirow[t]{2}{*}{$(B-15-1) 34 c c c-1$} & City of Cornish & 1961 & $u$ & 410 & 12 & 399 & $\mathbf{P}$ & 283-303 \\
\hline & & & & & & & & $325-330$ \\
\hline$(B-15-1) 34 c c c-2$ & Town of Cornish & - & $u$ & 32 & 12 & - & - & \\
\hline \multirow[t]{2}{*}{$13538 \mathrm{E} 03 \mathrm{DDB} 1$} & Millard, Wayne & 1961 & 1 & 382 & 16 & 382 & $P$ & $47-382$ \\
\hline & & & & & & & & $382-400$ \\
\hline 13S 38E 04AAB1 & Sorensen, Gene & 1962 & H & 94 & 8 & 94 & $\mathbf{P}$ & $30-94$ \\
\hline 13S 38E 04BDA 1 & Sorenson, Gene & - & 1 & - & - & - & - & \\
\hline
\end{tabular}




\begin{tabular}{|c|c|c|c|c|c|c|c|c|}
\hline \multirow{4}{*}{$\begin{array}{c}\text { Altitude } \\
\text { of land } \\
\text { surface } \\
\text { (feet) }\end{array}$} & \multicolumn{2}{|c|}{ Water level } & & & \multirow{2}{*}{\multicolumn{3}{|c|}{ Water-quality parameters }} & \multirow{4}{*}{$\begin{array}{c}\text { Other } \\
\text { data } \\
\text { available }\end{array}$} \\
\hline & \multirow{3}{*}{$\begin{array}{c}\text { Above (-) } \\
\text { or below } \\
\text { land surface } \\
\text { (feet) }\end{array}$} & \multirow[b]{3}{*}{ Date } & \multirow{2}{*}{\multicolumn{2}{|c|}{ Yield }} & & & & \\
\hline & & & & & & Specific & & \\
\hline & & & $\begin{array}{c}\text { Rate } \\
\text { (gal/min) }\end{array}$ & Date & $\begin{array}{c}\text { Temperature } \\
\left({ }^{\circ} \mathrm{C}\right)\end{array}$ & $\begin{array}{c}\text { conductance } \\
(\mu \mathrm{S} / \mathrm{cm})\end{array}$ & Date & \\
\hline 4,415 & -7.3 & $03-07-91$ & $4.0 \mathrm{~F}$ & 03-07-91 & 17.0 & 890 & 03-07-91 & W,C,D \\
\hline 4,415 & 4.88 & $03-07-91$ & - & - & - & - & - & \\
\hline 4,660 & - & - & - & - & - & - & - & L \\
\hline 4,527 & 22.22 & $03-18-91$ & $11 P$ & $07-15-88$ & - & - & - & \\
\hline 4,527 & 23.00 & $03-08-91$ & - & - & - & - & - & \\
\hline 4,410 & -7.2 & $03-21-91$ & $4.6 \mathrm{~F}$ & $03-21-91$ & 12.0 & 430 & $03-21-91$ & \\
\hline 4,425 & -37.1 & $03-26-91$ & $8.6 \mathrm{~F}$ & $03-26-91$ & 12.0 & 405 & $03-26-91$ & \\
\hline 4,425 & -12.8 & $03-22-91$ & $15 \mathrm{~F}$ & $03-22-91$ & 11.0 & 450 & $03-22-91$ & \\
\hline 4,419 & -7.2 & $03-22-91$ & - & - & 12.0 & 410 & $03-22-91$ & \\
\hline 4,420 & -12.0 & $03-07-91$ & $.6 \mathrm{~F}$ & $01-16-91$ & 12.0 & 580 & 03-07-91 & W,C,D \\
\hline 4,604 & 18.95 & 03-06-91 & - & - & - & - & - & \\
\hline 4,510 & -4.0 & 03-18-91 & $1 F$ & 03-18-91 & - & - & - & \\
\hline 4,485 & 4.87 & 03-07-91 & $7 P$ & 03-26-81 & - & - & - & $w$ \\
\hline 4,645 & 140.68 & $03-14-91$ & - & - & - & - & - & $L$ \\
\hline 4,620 & 96.64 & 03-14-91 & - & - & - & - & - & \\
\hline 4,420 & -2.2 & $03-14-91$ & $2.8 \mathrm{~F}$ & $03-14-91$ & 10.5 & 1,370 & $03-14-91$ & \\
\hline 4,415 & -43.8 & $03-14-91$ & $15.0 \mathrm{~F}$ & $03-14-91$ & 17.5 & 1,310 & $03-14-91$ & \\
\hline 4,460 & 11.68 & $03-07-91$ & $110 P$ & $05-05-70$ & - & - & - & $w$ \\
\hline 4,410 & -17.0 & $03-07-91$ & $.4 \mathrm{~F}$ & 03-07-91 & 10.5 & 600 & 03-07-91 & $W, D$ \\
\hline 4,445 & -0.5 & $03-27-91$ & - & - & - & - & - & \\
\hline 4,555 & 12.63 & $03-27-91$ & - & - & - & - & - & \\
\hline 4,548 & 16.98 & $03-27-91$ & - & - & - & - & - & \\
\hline 4,440 & -27.3 & $03-20-91$ & $15 \mathrm{~F}$ & 03-20-91 & 18.0 & 1,750 & $03-20-91$ & \\
\hline 4,480 & -3.8 & $03-15-91$ & - & - & - & - & - & \\
\hline 4,525 & 16.26 & $03-15-91$ & - & - & - & - & - & \\
\hline 4,555 & - & - & - & - & - & - & - & \\
\hline 4,640 & 47.63 & $03-19-91$ & $300 P$ & $12-06-79$ & - & - & - & \\
\hline 4,800 & 14.19 & $03-19-91$ & - & - & - & - & - & \\
\hline 4,790 & 11.35 & $03-04-87$ & - & - & - & - & - & $w$ \\
\hline 4,800 & -0.3 & $03-27-91$ & - & - & - & - & - & \\
\hline 4,890 & 15.95 & $03-07-91$ & - & - & - & - & - & $w$ \\
\hline 4,790 & 1.96 & $03-27-91$ & - & - & - & - & - & \\
\hline 4,510 & 9.74 & $03-07-91$ & - & - & - & - & - & $w$ \\
\hline 4,515 & 11.05 & $03-07-91$ & - & - & - & - & - & $w$ \\
\hline 4,850 & 23.45 & $03-07-91$ & - & - & - & - & - & $w$ \\
\hline 4,800 & 30.98 & $03-11-91$ & - & - & - & - & - & \\
\hline 4,790 & - & - & $190 P$ & $07-31-90$ & - & - & - & \\
\hline
\end{tabular}


Table 1.-Records of

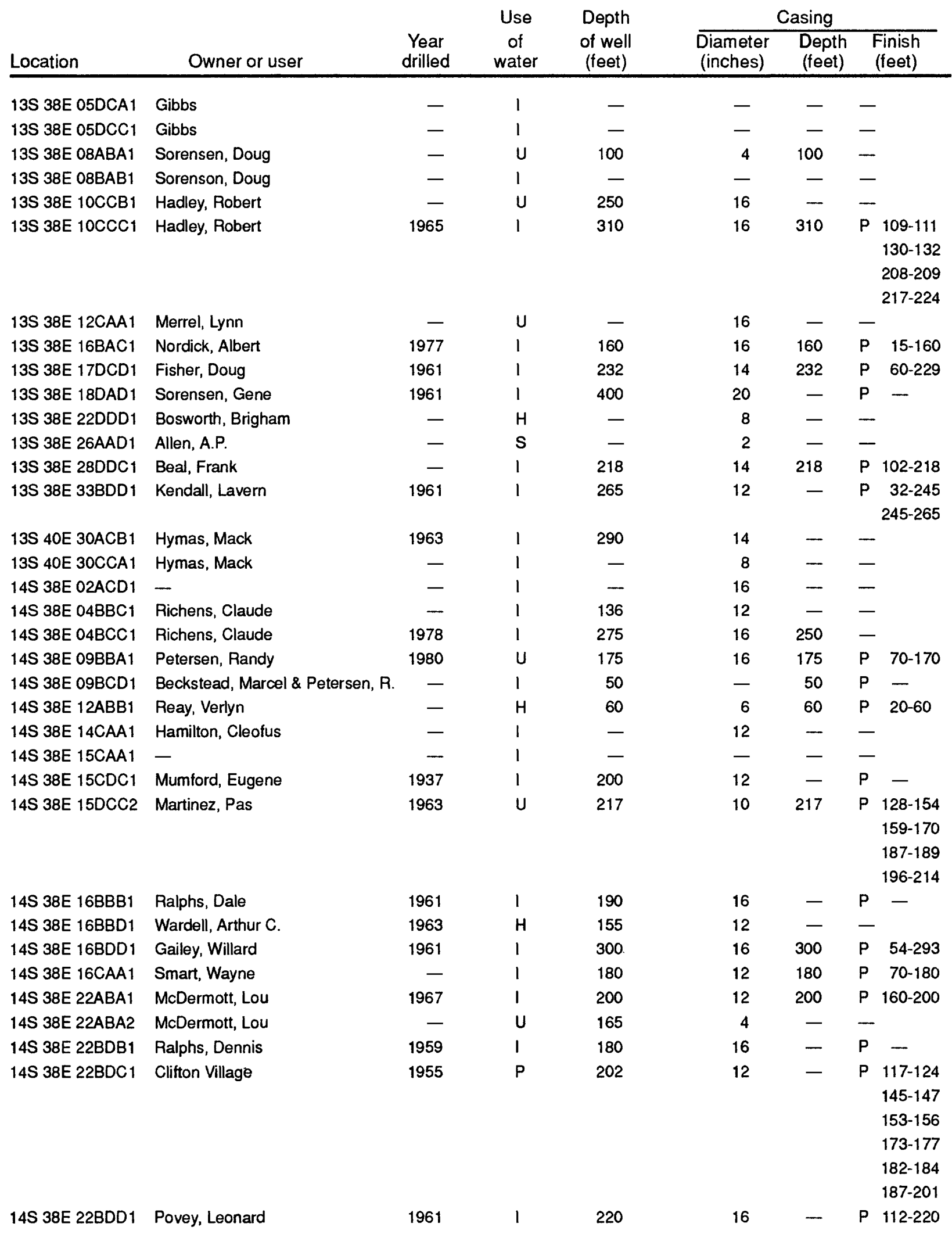




\begin{tabular}{|c|c|c|c|c|c|c|c|c|}
\hline \multirow{3}{*}{$\begin{array}{c}\text { Altitude } \\
\text { of land } \\
\text { surface } \\
\text { (feet) }\end{array}$} & \multicolumn{2}{|c|}{ Water level } & & & \multirow{2}{*}{\multicolumn{3}{|c|}{ Water-quality parameters }} & \multirow{3}{*}{$\begin{array}{c}\text { Other } \\
\text { data } \\
\text { available }\end{array}$} \\
\hline & Above (-) & & & & & & \\
\hline & $\begin{array}{c}\text { or below } \\
\text { land surface } \\
\text { (feet) }\end{array}$ & Date & $\frac{\text { Yield }}{\text { Rate }}$ & Date & $\begin{array}{c}\text { Temperature } \\
\left({ }^{\circ} \mathrm{C}\right)\end{array}$ & $\begin{array}{c}\text { Specific } \\
\text { conductance } \\
(\mu \mathrm{S} / \mathrm{cm})\end{array}$ & Date & \\
\hline 4,910 & - & - & - & - & - & - & - & \\
\hline 4,930 & - & - & $300 \mathrm{P}$ & $07-31-90$ & - & - & - & \\
\hline 4,920 & 65.34 & $03-11-91$ & - & - & - & - & - & \\
\hline 5,060 & - & - & $2,000 \mathrm{P}$ & $08-01-90$ & - & - & - & \\
\hline 4,840 & 25.60 & $03-11-91$ & - & - & - & - & - & \\
\hline 4,800 & - & - & - & - & - & - & - & \\
\hline 5,120 & 49.06 & $03-12-91$ & - & - & - & - & - & \\
\hline 4,860 & - & - & - & - & - & - & - & \\
\hline 5,040 & - & - & - & - & - & - & - & \\
\hline 5,240 & - & - & $1,030 \mathrm{P}$ & $08-01-90$ & - & - & - & \\
\hline 4,860 & 31.00 & $03-19-91$ & - & - & - & - & - & \\
\hline 4,760 & -17.5 & $03-27-91$ & $12.0 \mathrm{~F}$ & $03-27-91$ & 12.0 & 1,060 & $03-27-91$ & \\
\hline 4,780 & .99 & $03-14-91$ & - & - & - & - & - & \\
\hline 4,785 & 16.40 & $03-14-91$ & $550 P$ & $08-02-90$ & - & - & - & $L$ \\
\hline 5,050 & 34.56 & $03-07-91$ & $670 P$ & $07-31-90$ & - & - & - & w \\
\hline 5,020 & - & - & - & - & - & 一 & - & \\
\hline 4,750 & 10.25 & 03-19-91 & - & - & - & - & - & \\
\hline 4,800 & 12.28 & 03-07-91 & - & - & - & - & - & $w$ \\
\hline 4,840 & - & - & $1,000 P$ & $08-02-90$ & - & - & - & \\
\hline 4,820 & 77.05 & $03-14-91$ & - & - & - & - & - & \\
\hline 4,780 & 45.44 & $03-14-91$ & - & - & - & - & - & \\
\hline 4,750 & 1.69 & 03-19-91 & - & - & - & - & - & \\
\hline 4,750 & - & - & - & - & - & - & - & \\
\hline 4,760 & - & - & $610 \mathrm{P}$ & $08-02-90$ & - & - & - & \\
\hline 4,800 & 33.91 & 03-07-91 & $490 \mathrm{P}$ & $08-02-90$ & - & - & - & $w$ \\
\hline 4,780 & - & - & - & - & - & - & - & \\
\hline 4,835 & - & - & $60.0 \mathrm{~F}$ & $04-12-90$ & - & - & - & \\
\hline 4,840 & - & - & $40.0 \mathrm{~F}$ & $04-12-90$ & - & - & - & \\
\hline 4,840 & - & - & - & - & - & - & - & \\
\hline 4,840 & 68.45 & $03-14-91$ & - & - & - & - & - & \\
\hline 4,750 & 7.75 & $03-14-91$ & - & - & - & - & - & \\
\hline 4,760 & - & - & - & - & - & - & - & \\
\hline 4,795 & 45.66 & $04-04-91$ & - & - & - & - & - & \\
\hline 4,840 & 77.22 & $04-04-91$ & - & - & - & - & - & \\
\hline
\end{tabular}


Table 1.-Records of

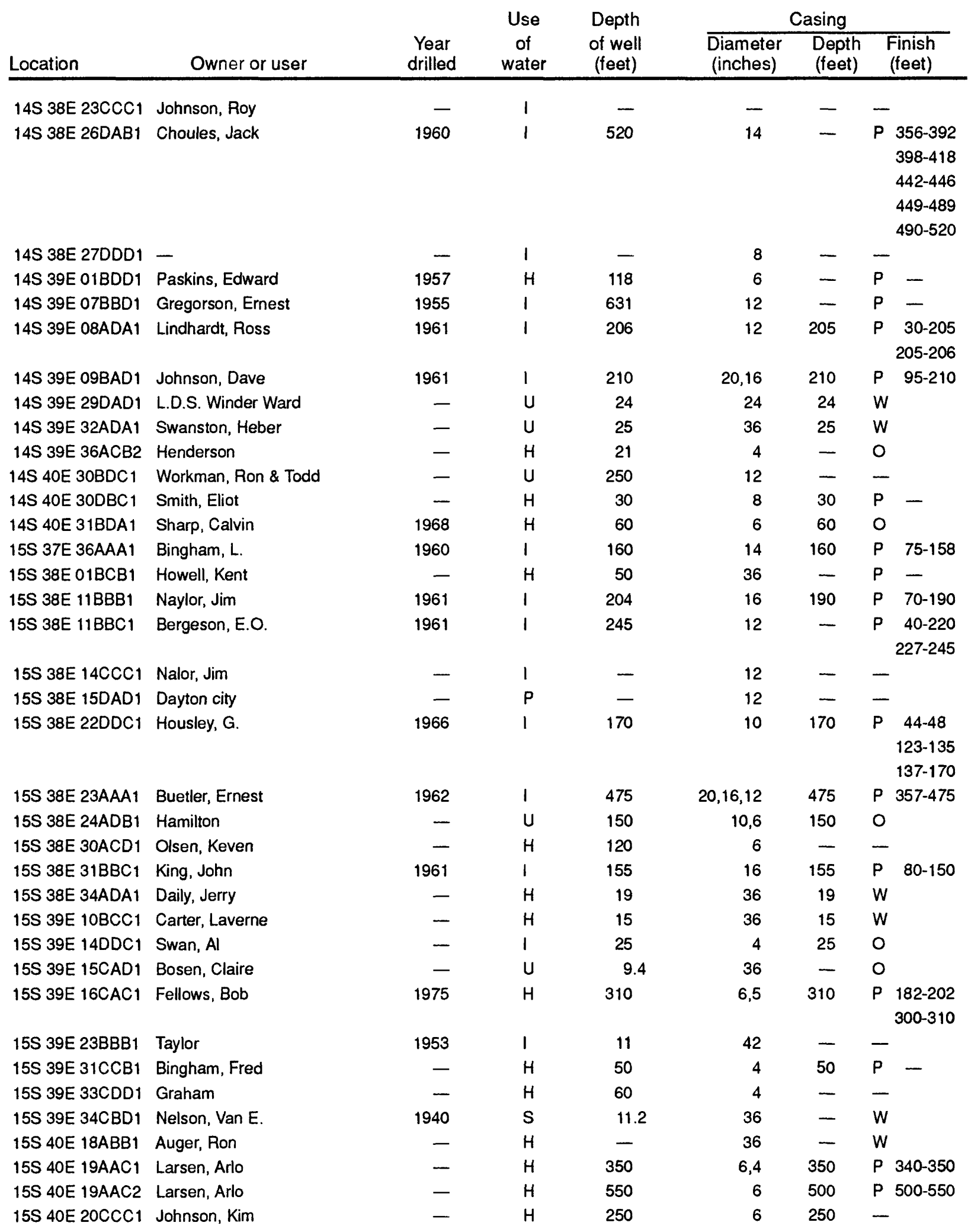




\begin{tabular}{|c|c|c|c|c|c|c|c|c|}
\hline \multirow{3}{*}{$\begin{array}{c}\text { Altitude } \\
\text { of land } \\
\text { surface } \\
\text { (feet) }\end{array}$} & \multicolumn{2}{|c|}{ Water level } & & & \multirow{2}{*}{\multicolumn{3}{|c|}{ Water-quality parameters }} & \multirow{3}{*}{$\begin{array}{c}\text { Other } \\
\text { data } \\
\text { available }\end{array}$} \\
\hline & Above (-) & & & & & & \\
\hline & $\begin{array}{c}\text { or below } \\
\text { land surface } \\
\text { (feet) }\end{array}$ & Date & $\frac{\text { Yiel }}{\text { Rate }}$ & Date & $\begin{array}{c}\text { Temperature } \\
\left({ }^{\circ} \mathrm{C}\right)\end{array}$ & $\begin{array}{c}\text { Specific } \\
\text { conductance } \\
(\mu S / \mathrm{cm})\end{array}$ & Date & \\
\hline 4,780 & 39.24 & $03-14-91$ & - & - & - & - & - & \\
\hline 4,745 & 14.85 & $03-14-91$ & - & - & - & - & - & \\
\hline 4,760 & 7.20 & $03-15-91$ & - & - & - & - & - & \\
\hline 5,050 & 48.73 & $03-27-91$ & - & - & - & - & - & \\
\hline 4,750 & 3.53 & $03-12-91$ & $270 P$ & $07-31-90$ & - & - & - & \\
\hline 4,850 & 86.48 & $03-08-91$ & - & - & - & - & - & w \\
\hline 4,885 & - & - & - & - & - & - & - & \\
\hline 4,755 & 6.98 & $03-08-91$ & - & - & - & - & - & $w$ \\
\hline 4,750 & 19.96 & $03-12-91$ & - & - & - & - & - & \\
\hline 4,540 & 18.30 & $03-19-91$ & - & - & - & - & - & \\
\hline 4,760 & 113.45 & $03-27-91$ & - & - & - & - & - & \\
\hline 4,560 & 8.98 & $03-19-91$ & - & - & - & - & - & \\
\hline 4,580 & 26.68 & $03-19-91$ & - & - & - & - & - & \\
\hline 5,075 & 74.09 & $03-22-91$ & $960 P$ & $08-02-90$ & - & - & - & \\
\hline 4,740 & 24.68 & $03-15-91$ & - & - & - & - & - & \\
\hline 4,800 & 65.53 & $03-15-91$ & - & - & - & - & - & \\
\hline 4,800 & - & - & $630 P$ & $08-02-90$ & - & - & - & \\
\hline 4,820 & 54.79 & $03-15-91$ & - & - & - & - & - & \\
\hline 4,815 & $57.50 \mathrm{R}$ & 03-13-91 & - & - & - & - & - & \\
\hline 4,760 & 25.85 & $03-07-91$ & 一 & - & - & - & - & $w$ \\
\hline 4,750 & 57.36 & 03-15-91 & - & - & - & - & - & \\
\hline 4,735 & 87.20 & $03-15-91$ & - & - & - & - & - & \\
\hline 4,160 & 22.10 & $04-04-91$ & - & - & - & - & - & \\
\hline 5,060 & - & - & $1,330 P$ & $08-02-90$ & - & - & - & \\
\hline 4,770 & 13.38 & $03-22-91$ & - & - & - & - & - & \\
\hline 4,730 & 12.45 & $03-20-91$ & - & - & - & - & - & \\
\hline 4,730 & 4.45 & $03-21-91$ & - & - & - & - & - & \\
\hline 4,720 & 6.20 & $03-08-91$ & - & - & - & - & - & $w$ \\
\hline 4,565 & 一 & - & $30 \mathrm{P}$ & $07-08-75$ & - & - & - & $L$. \\
\hline $4,725.4$ & 4.14 & $09-05-84$ & - & - & - & - & - & $w$ \\
\hline 4,700 & 11.95 & $03-21-91$ & - & - & - & - & - & \\
\hline 4,660 & 24.97 & $03-26-91$ & - & - & - & - & - & \\
\hline 4,650 & 7.00 & $03-09-70$ & - & - & - & - & - & $w$ \\
\hline 4,860 & $8.00 \mathrm{R}$ & $03-20-91$ & - & - & - & - & - & \\
\hline 4,860 & 203.38 & $03-27-91$ & - & - & - & - & - & \\
\hline 4,860 & 195.98 & $03-27-91$ & - & - & - & - & - & \\
\hline 4,800 & 109.38 & $03-20-91$ & - & - & - & - & - & \\
\hline
\end{tabular}


Table 1.-Records of

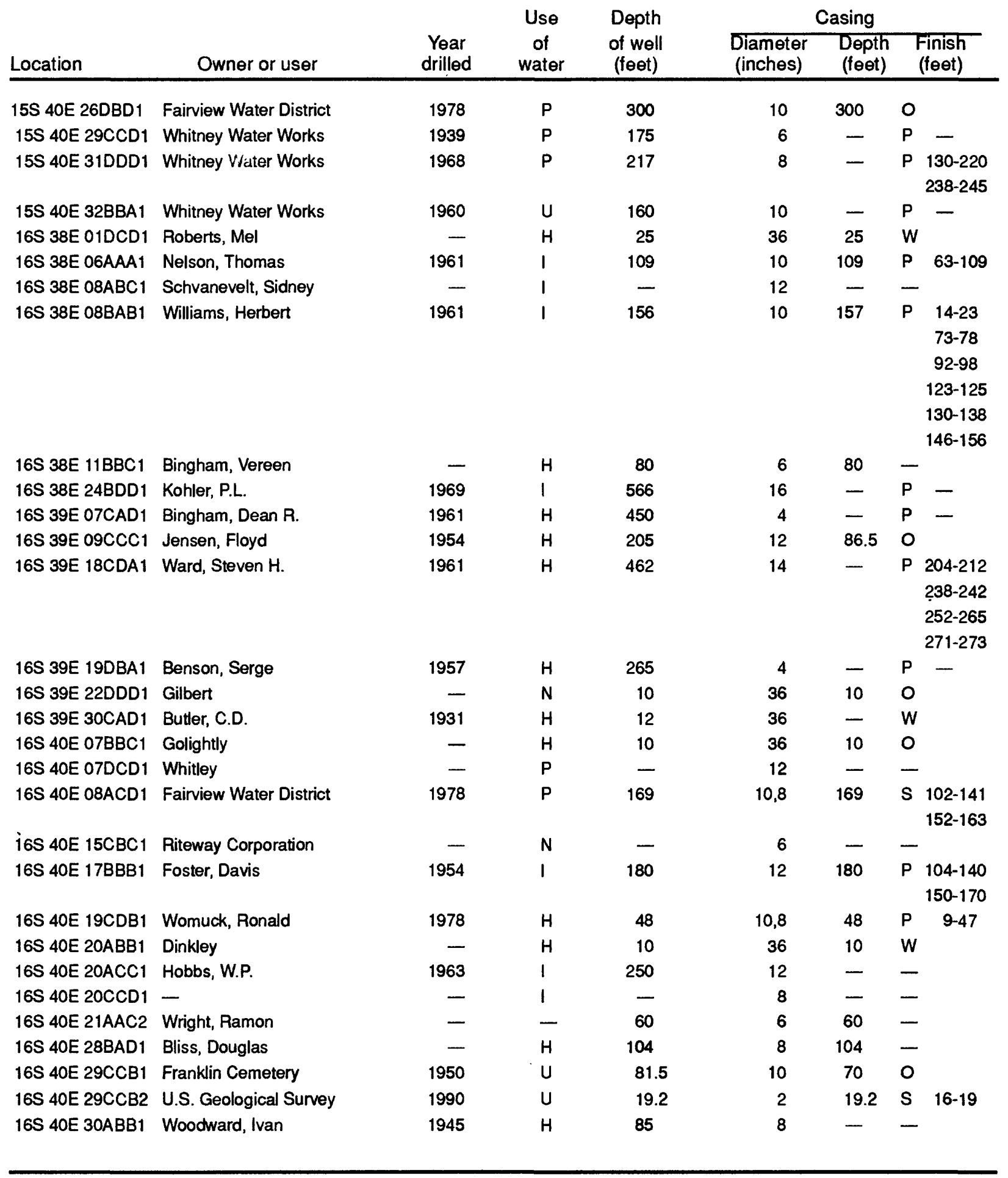




\begin{tabular}{|c|c|c|c|c|c|c|c|c|}
\hline \multirow{3}{*}{$\begin{array}{c}\text { Altitude } \\
\text { of land } \\
\text { surface } \\
\text { (feet) }\end{array}$} & \multicolumn{2}{|c|}{ Water level } & & & \multirow{2}{*}{\multicolumn{3}{|c|}{ Water-quality parameters }} & \multirow{3}{*}{$\begin{array}{c}\text { Other } \\
\text { data } \\
\text { available }\end{array}$} \\
\hline & \multirow{2}{*}{$\begin{array}{c}\text { Above (-) } \\
\text { or below } \\
\text { land surface } \\
\text { (feet) }\end{array}$} & \multirow[b]{2}{*}{ Date } & & & & & \\
\hline & & & 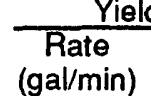 & $\overline{\text { Date }}$ & $\begin{array}{c}\text { Temperature } \\
\left({ }^{\circ} \mathrm{C}\right)\end{array}$ & $\begin{array}{l}\text { Specific } \\
\text { conductance } \\
(\mu \mathrm{S} / \mathrm{cm})\end{array}$ & Date & \\
\hline 4,700 & - & - & $70 \mathrm{P}$ & $03-21-78$ & - & - & - & L \\
\hline 4,750 & - & - & - & - & - & - & - & \\
\hline 4,680 & - & - & - & - & - & - & - & \\
\hline 4,735 & 1.51 & $03-20-91$ & - & - & - & - & - & \\
\hline 4,635 & 12.03 & 03-22-91 & - & - & - & - & - & \\
\hline 5,020 & 29.05 & $04-04-91$ & - & - & - & - & - & \\
\hline 4,940 & - & - & $1,220 P$ & $08-02-90$ & - & - & - & \\
\hline 4,940 & 2.44 & $04-04-91$ & $130 P$ & $08-02-90$ & - & - & - & \\
\hline 4,760 & .71 & $03-22-91$ & - & - & - & - & - & \\
\hline 4,585 & 58.81 & $03-21-91$ & $2,500 \mathrm{P}$ & $06-00-69$ & - & - & - & \\
\hline 4,550 & 58.50 & $03-21-91$ & - & - & - & - & - & \\
\hline 4,550 & 4.62 & 03-27-91 & - & - & - & - & - & \\
\hline $4,542.7$ & 27.85 & $03-07-91$ & - & - & - & - & - & $L, W$ \\
\hline 4,550 & 39.75 & $03-21-91$ & - & - & - & - & - & \\
\hline 4,515 & 4.30 & 03-26-91 & - & - & - & - & - & \\
\hline 4,550 & 11.94 & $03-21-91$ & - & - & - & - & - & \\
\hline 4,655 & 3.50 & $03-26-91$ & - & - & - & - & - & \\
\hline 4,545 & - & - & - & - & - & - & - & \\
\hline 4,640 & - & - & $160 P$ & $03-23-78$ & - & - & - & $L$ \\
\hline 4,620 & 52.36 & 03-25-91 & - & - & - & - & - & \\
\hline 4,550 & 14.52 & $03-20-91$ & $240 P$ & $07-30-90$ & - & - & - & \\
\hline 4,520 & - & - & $68 \mathrm{P}$ & $06-28-78$ & - & - & - & \\
\hline 4,520 & 6.42 & 03-26-91 & - & - & - & - & - & \\
\hline 4,480 & - & - & - & - & - & - & - & \\
\hline 4,495 & - & - & - & - & - & - & - & \\
\hline 4,560 & 10.21 & $03-25-91$ & - & - & - & - & - & \\
\hline 4,450 & -5.9 & $03-27-91$ & - & - & 9.5 & 540 & 03-27-91 & \\
\hline $4,504.9$ & 22.44 & $03-08-91$ & - & - & - & - & - & w \\
\hline 4,500 & 13.15 & 03-08-91 & - & - & - & - & - & \\
\hline 4,500 & 0 & $03-26-91$ & - & - & - & - & - & \\
\hline
\end{tabular}




\section{Table 2._Drillers' logs of selected wells}

Location: See page 2 for an explanation of the numbering systems for hydrologic-data sites.

Altitude: Altitude of land surface, in feet above sea level.

Thickness: In feet.

Depth: Depth to bottom of interval, in feet below land surface.

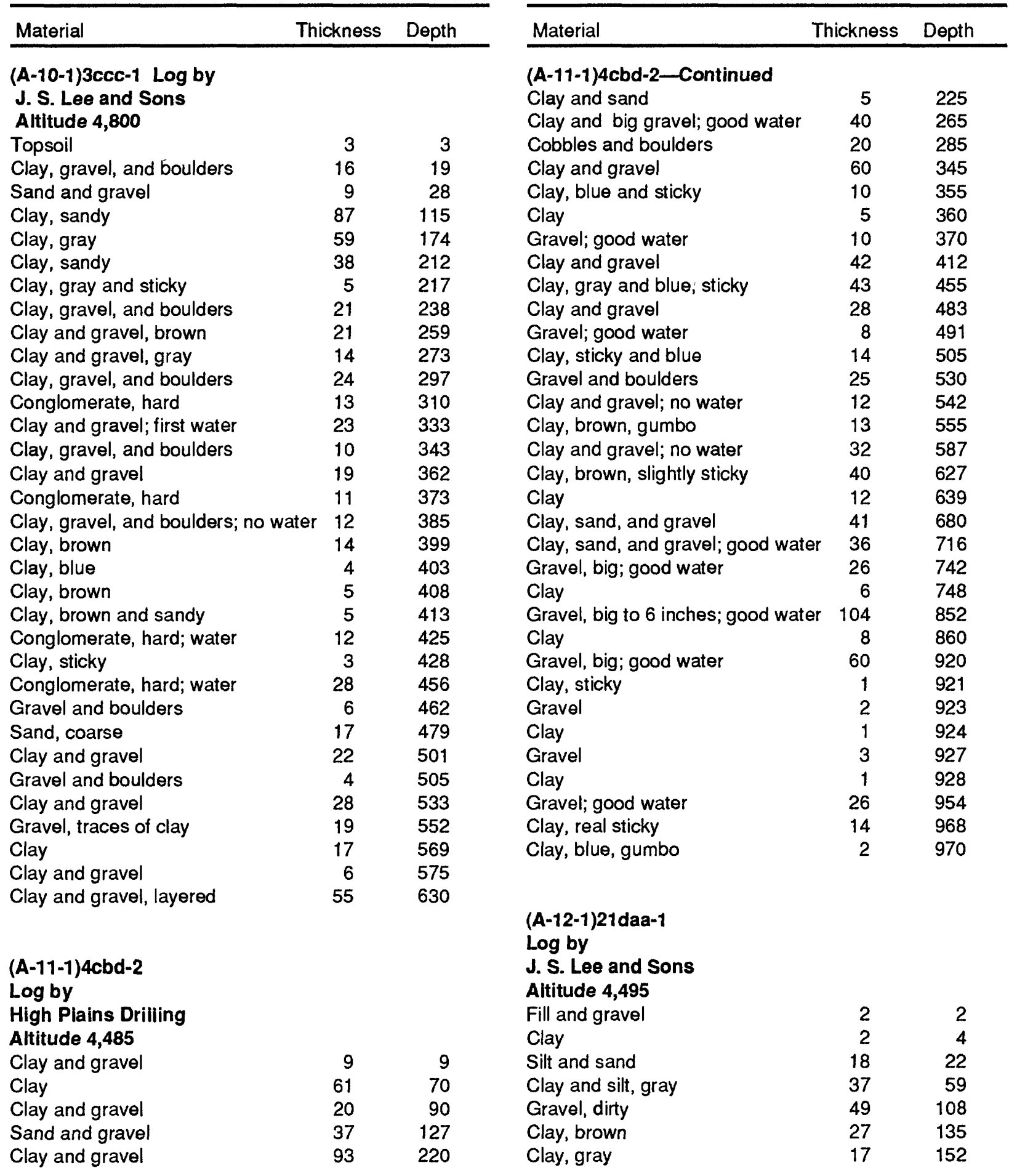


Table 2.-Drillers' logs of selected wells-Continued

\begin{tabular}{|c|c|c|c|c|c|}
\hline Material & Thickness & Depth & Material & Thickness & Depth \\
\hline \multicolumn{3}{|l|}{ (A-12-1)21daa-1-Continued } & \multicolumn{3}{|l|}{ (A-12-1)35bcc-1-Continued } \\
\hline Gravel; water (flow); iron content & 28 & 180 & Clay & 6 & 756 \\
\hline Clay and gravel & 24 & 204 & Gravel, hard & 4 & 760 \\
\hline Gravel; water & 14 & 218 & Clay and boulders & 15 & 775 \\
\hline Clay and gravel & 8 & 226 & Gravel, hard & 10 & 785 \\
\hline Conglomerate & 25 & 251 & Clay, gummy & 3 & 788 \\
\hline Clay and gravel & 21 & 272 & Gravel, hard, cemented & 27 & 815 \\
\hline Clay & 12 & 284 & Clay & 5 & 820 \\
\hline Conglomerate & 15 & 299 & Silt and gravel, some hard & & \\
\hline Gravel; water & 4 & 303 & cemented, some loose & 78 & 898 \\
\hline Clay and gravel & 4 & 307 & Clay, sand, and gravel, & & \\
\hline Conglomerate, hard & 18 & 325 & $\operatorname{coars} \theta$, hard, sticky & 80 & 978 \\
\hline Clay, blue and sticky & 21 & 346 & & & \\
\hline Conglomerate, hard & 54 & 400 & & & \\
\hline Gravel; water & 23 & $423^{\circ}$ & $(A-14-1) 23 b d d-1$ & & \\
\hline Conglomerate, hard & 7 & 430 & Log by & & \\
\hline \multirow[t]{2}{*}{ Clay, gray and sticky } & 14 & 444 & $\begin{array}{l}\text { J. S. Lee and Sons } \\
\text { Altitude } 4,600\end{array}$ & & \\
\hline & & & Gravel and boulders & 14 & 14 \\
\hline$(A-12-1) 35 b c c-1$ & & & Clay and gravel & 6 & 20 \\
\hline Log by & & & Clay, gravel, and boulders & 45 & 65 \\
\hline Technical Services Inc. & & & Clay, gray & 37 & 102 \\
\hline Altitude 4,600 & & & Clay and gravel & 10 & 112 \\
\hline Gravel fill & 4 & 4 & Clay, gray & 339 & 451 \\
\hline Silt, muddy & 6 & 10 & Gravel & 14 & 465 \\
\hline Silt and gravel; little water & 8 & 18 & Clay, gray & 138 & 603 \\
\hline Clay, brown, and sand, gray & 64 & 82 & & & \\
\hline Clay, silt and gravel & 10 & 92 & & & \\
\hline Silt and gravel, hard & 34 & 126 & (A-14-1)34add-1 & & \\
\hline Clay and gravel, brown and sandy & 46 & 172 & Log by & & \\
\hline \multicolumn{6}{|l|}{ Clay and gravel, sandy, some } \\
\hline sediment; little water & 32 & 211 & Clay & 13 & 13 \\
\hline Clay with hard rock & 24 & 235 & Boulders & 52 & 65 \\
\hline Brown mud and hard rock & 49 & 284 & Gravel and boulders; water & 40 & 105 \\
\hline Clay with hard rock & 6 & 290 & Clay, $\tan$ & 13 & 118 \\
\hline Clay, silt, and gravel & 16 & 306 & Clay and gravel, gray & 25 & 143 \\
\hline Sand and gravel; good water & 16 & 322 & Clay and gravel, rust colored & 7 & 150 \\
\hline \multirow{2}{*}{$\begin{array}{l}\text { Silt and gravel, hard; little } \\
\text { water }\end{array}$} & & & Conglomerate & 35 & 185 \\
\hline & 17 & 339 & Clay and boulders, red & 7 & 192 \\
\hline Clay, silty, gummy & 45 & 384 & Conglomerate and bedrock & 35 & 227 \\
\hline Broken rock & 4 & 388 & Bedrock, fractured limestone & 29 & 256 \\
\hline Clay, silt, and gravel, hard & 28 & 416 & Clay and gravel, light blue & 18 & 274 \\
\hline \multirow{2}{*}{$\begin{array}{l}\text { Clay and gravel, very hard; } \\
\text { some water }\end{array}$} & & & Clay and gravel, in alternating & & \\
\hline & 38 & 454 & strata & 21 & 295 \\
\hline Clay, silty, sandy and gummy & 50 & 504 & Clay, light blue & 32 & 327 \\
\hline Clay and gravel & 6 & 510 & Clay and gravel, blue & 16 & 343 \\
\hline Gravel, hard & 14 & 524 & Bedrock, black limestone & 30 & 373 \\
\hline Clay, gummy and sandy & 64 & 588 & Clay and gravel & 7 & 380 \\
\hline \multirow{3}{*}{$\begin{array}{l}\text { Silt, gravel, and boulders, } \\
\text { hard, some clay streak; } \\
\text { some water }\end{array}$} & & & Bedrock, black limestone & 17 & 397 \\
\hline & & & Bedrock, fractured limestone & 38, & 435 \\
\hline & 162 & 750 & & & \\
\hline
\end{tabular}




\section{Table 2.-Drillers' logs of selected wells-Continued}

\begin{tabular}{lcc}
\hline Material & Thickness & Depth \\
\hline $\begin{array}{l}\text { (A-14-1)34add-1-Continued } \\
\text { Bedrock, fractured limestone }\end{array}$ & & \\
interbedded with soft blue limestone & 2 & 437 \\
$\begin{array}{l}\text { Bedrock, fractured limestone } \\
\text { Bedrock, soft and hard }\end{array}$ & 6 & 443 \\
limestone interbedded & 15 & 458 \\
Bedrock, fractured limestone & 46 & 504
\end{tabular}

\section{(A-15-2)31 cba-1}

Log by

R. G. Harding

Altitude 4,620

Boulders and soil

Boulders and gravel; small flow

Clay, sandy, gray; with little

quartzite grit

Conglomerate

Clay, sandy

Clay with small amount of gravel

Clay, blue

Clay with small amount of gravel

Clay, gray

Clay, gray

Clay with coarse sand

Clay, fine and sandy

Clay and gravel, gray

Clay, gray

\begin{tabular}{ll}
\hline Material Thickness Depth \\
\hline
\end{tabular}

(B-10-1)3aab-1-Continued

Gravel; water

$31 \quad 385$

Clay, black

$8 \quad 393$
(B-11-1)14cda-1

Log by

Wright Drilling Co.

Altitude 4,440

Topsoil with clay

Clay, brown

Clay, blue

Clay and gravel, blue

Sand and gravel; water

Clay and gravel

Sand and gravel; water

Clay and gravel

Gravel; water

Clay, gray

Clay and sand

Sand; water

Gravel; water

Clay and gravel

Sand; water

Clay and gravel

Clay and sand

Sand and gravel; water

Clay and gravel; water

(B-12-1)19cca-1

Log by

Rex L. Frandsen

Altitude 4,660

Clay and cobbles

Clay, gravel, cobbles, and

boulders

Bedrock, shale and limestone

Clay, gravel, cobbles, and

boulders

Boulders

Clay and cobbles, green clay

Boulders

Clay and cobbles

Clay and gravel; water

Clay and cobbles

Clay, gravel, and cobbles

Boulders

Clay, gravel, and cobbles

\section{3}

65

17

35

120

$4 \quad 124$

$51 \quad 175$

$4 \quad 179$

$21 \quad 200$

$40 \quad 240$

$20 \quad 260$

$58 \quad 318$

4322

Clay and gravel, brown

Gravel; water

Clay, brown

Gravel; water

Clay, brown

339

354 


\section{Table 2.-Drillers' logs of selected wells-Continued}

\begin{tabular}{|c|c|c|c|c|c|}
\hline Material & Thickness & Depth & Material & Thickness & Depth \\
\hline \multicolumn{3}{|l|}{$(B-13-1) 16 c b d-1$} & \multicolumn{3}{|l|}{ 15S 39E 16CAC1-Continued } \\
\hline Log by & & & Shale, soft and blue & 80 & 160 \\
\hline T. J. Smith & & & Sand, fine; water & 20 & 180 \\
\hline Altitude 4,645 & & & Shale, soft and gray & 20 & 200 \\
\hline ( 0 to 3 feet not reported) & 3 & 3 & Clay, sandy & 20 & 220 \\
\hline Clay, brown & 30 & 33 & Shale, hard and gray & 20 & 240 \\
\hline Sand, fine & 7 & 40 & Sand, fine; water & 30 & 270 \\
\hline Clay and streaks of hardpan & 20 & 60 & Shale, gray; water & 10 & 280 \\
\hline \multicolumn{3}{|l|}{ Sand and gravel, fine; little } & Shale & 25 & 305 \\
\hline water & 20 & 80 & Gravel and sand, coarse; water & 5 & 310 \\
\hline Clay, blue & 10 & 90 & & & \\
\hline Sand, black & 1 & 91 & & & \\
\hline Clay & 29 & 120 & 15S 40E 26DBD1 & & \\
\hline Clay, blue & 5 & 125 & Log by & & \\
\hline Sand & 25 & 150 & W. E. Stevens and Sons & & \\
\hline Clay, yellow & 25 & 175 & Altitude 4,700 & & \\
\hline Sand; small flow & 25 & 200 & Topsoil, gravel, and boulders; water & Fr 10 & 10 \\
\hline Clay, yellow & 5 & 205 & Clay and boulders, brown & 5 & 15 \\
\hline \multirow[t]{3}{*}{ Sand } & 5 & 210 & Clay, blue & 2 & 17 \\
\hline & & & Shale with soft spots, blue; water & 91 & 108 \\
\hline & & & Shale, green and yellow; water & 4 & 112 \\
\hline 13S 38E 33BDD1 & & & Shale, blue & 20 & 132 \\
\hline Log by & & & Shale, green and yellow & 3 & 135 \\
\hline Ivan Bortz & & & Shale, blue & 5 & 140 \\
\hline Altitude 4,785 & & & Shale, green & 5 & 145 \\
\hline Topsoil & 6 & 6 & Shale, blue & 8 & 153 \\
\hline Clay, blue & 14 & 20 & Shale, green & 7 & 160 \\
\hline Clay, red and sandy & 8 & 28 & Shale, blue & 140 & 300 \\
\hline Clay, blue & 10 & 38 & & & \\
\hline Shale; water & 1 & 39 & & & \\
\hline Gravel, clay, and rocks; water & 3 & 42 & 16S 39E 18CDA1 & & \\
\hline Clay, yellow; water & 11 & 53 & Log by & & \\
\hline Sandstone, green; water & 16 & 69 & Charles B. Gardner & & \\
\hline Rocks; water & 2 & 71 & Altitude $4,542.7$ & & \\
\hline Shale, rocks, and sandstone; water & r 129 & 200 & Topsoil & 2 & 2 \\
\hline Shale, blue clay in layers; water & 5 & 205 & Clay, gravelly & 14 & 16 \\
\hline Shale rock or sandstone; water & 30 & 235 & Sand & 10 & 26 \\
\hline Rock, blue & 20 & 255 & Clay, blue & 14 & 40 \\
\hline Shale rock or sandstone and & & & Clay, yellow & 40 & 80 \\
\hline \multirow[t]{3}{*}{ clay, blue } & 10 & 265 & Clay, blue & 63 & 143 \\
\hline & & & Clay, gravelly & 5 & 148 \\
\hline & & & Clay, blue & 2 & 150 \\
\hline 15S 39E 16CAC1 & & & Clay, gravelly & 6 & 156 \\
\hline Log by & & & Clay, blue & 48 & 204 \\
\hline Ivan Bortz & & & Clay, gravelly; water & 8 & 212 \\
\hline Altitude 4,565 & & & Clay, blue & 26 & 238 \\
\hline Topsoil & 2 & 2 & Gravel; water & 4 & 242 \\
\hline Sand, gravel, and clay; water & 16 & 18 & Clay, blue & 10 & 252 \\
\hline Sand; water & 3 & 21 & Gravel; water & 13 & 265 \\
\hline Clay, hard and yellow; water & 1 & 22 & Clay, blue & 6 & 271 \\
\hline Sand, very fine & 23 & 45 & Gravel; water & 2 & 273 \\
\hline Clay, sandy and blue & 35 & 80 & Clay, blue & 18 & 291 \\
\hline
\end{tabular}


Table 2.-Drillers' logs of selected wells-Continued

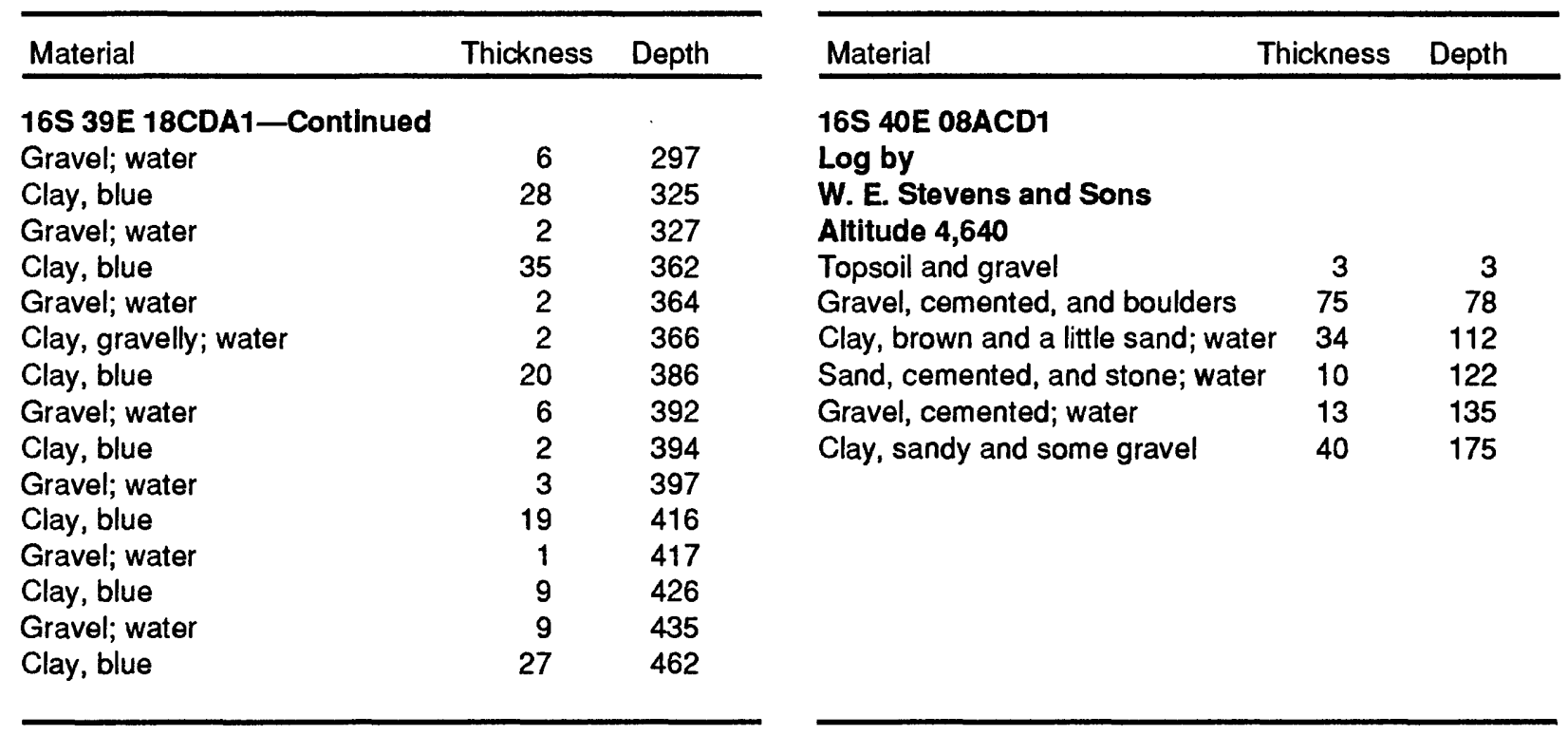


Table 3.-Water levels in selected wells

Location: See page 2 for an explanation of the numbering systems for hydrologic-data sites. Altitude: Altitude of land surface, in feet above sea level.

Water levels are in feet below or above (-) land surface.

\begin{tabular}{|c|c|c|c|c|c|}
\hline Date & Water level & Date & Water level & Date & Water level \\
\hline \multicolumn{2}{|l|}{$(A-10-1) 16$ dad-1 } & \multicolumn{2}{|c|}{ (A-10-1)16dad-1-Continued } & \multicolumn{2}{|c|}{ (A-11-1)4daa-2-Continued } \\
\hline Altitude 4,820 & & MAR 01, 1990 & 10.29 & JAN 25,1970 & 14.11 \\
\hline AUG 05,1969 & 6.21 & APR 27 & 8.01 & 31 & 14.20 \\
\hline SEP 04 & 6.85 & MAY 29 & 7.96 & FEB 05 & 14.39 \\
\hline ОСТ 09 & 8.15 & JUN 26 & 7.90 & 10 & 14.60 \\
\hline NOV 07 & 10.13 & JUL 26 & 8.45 & 15 & 14.78 \\
\hline DEC 02 & 10.46 & AUG 28 & 9.70 & 20 & 14.95 \\
\hline JAN 07,1970 & 10.65 & SEP 27 & 10.25 & 25 & 15.13 \\
\hline FEB 09 & 10.43 & ОСТ 30 & 10.39 & 28 & 15.09 \\
\hline MAR 04 & 10.43 & NOV 28 & 10.48 & MAR 05 & 15.30 \\
\hline MAR 12,1971 & 10.23 & JAN 03, 1991 & 10.62 & 10 & 15.60 \\
\hline SEP 01 & 4.73 & MAR 08 & 10.21 & 15 & 15.60 \\
\hline MAR 01,1972 & 10.23 & & & 20 & 15.81 \\
\hline SEP 12 & 5.19 & (A-11-1)4daa-2 & & 25 & 15.92 \\
\hline MAR 02, 1973 & 10.35 & Altitude $\mathbf{4 , 5 0 0}$ & & SEP 03 & 15.77 \\
\hline SEP 13 & 4.80 & AUG 05,1969 & 13.04 & MAR 01, 1971 & 15.50 \\
\hline MAR 13, 1974 & 9.53 & 10 & 14.71 & SEP 01 & 9.46 \\
\hline SEP 11 & 5.20 & 15 & 15.00 & MAR 01, 1972 & 13.08 \\
\hline MAR 11, 1975 & 10.26 & 25 & 16.03 & SEP 12 & 8.40 \\
\hline OCT 15 & 7.73 & 31 & 16.37 & MAR 02, 1973 & 13.79 \\
\hline MAR 15, 1976 & 10.09 & SEP 05 & 15.74 & SEP 13 & 12.36 \\
\hline SEP 13 & 6.29 & 10 & 15.40 & MAR 13, 1974 & 15.79 \\
\hline MAR 08, 1977 & 10.48 & 15 & 13.80 & SEP 11 & 11.31 \\
\hline SEP 28 & 10.06 & 20 & 13.30 & MAR 12, 1975 & 14.10 \\
\hline MAR 08, 1978 & 10.53 & 25 & 13.07 & OCT 15 & 9.16 \\
\hline SEP 18 & 5.83 & .30 & 12.31 & MAR 15,1976 & 13.65 \\
\hline MAR 12, 1979 & 9.88 & OCT 05 & 11.98 & SEP 13 & 10.11 \\
\hline SEP 17 & 5.86 & 10 & 11.68 & MAR 08, 1977 & 14.29 \\
\hline MAR 10, 1980 & 10.11 & 15 & 11.62 & SEP 28 & 19.42 \\
\hline SEP 17 & 5.76 & 20 & 11.72 & MAR 08, 1978 & 19.54 \\
\hline MAR 02, 1981 & 10.49 & 25 & 11.60 & SEP 18 & 15.93 \\
\hline SEP 17 & 8.28 & 31 & 11.64 & MAR 12, 1979 & 17.30 \\
\hline MAR 08, 1982 & 10.16 & NOV 05 & 11.50 & SEP 17 & 16.48 \\
\hline SEP 08 & 6.07 & 10 & 11.69 & MAR 10,1980 & 17.79 \\
\hline MAR 02, 1983 & 10.22 & 15 & 11.69 & SEP 17 & 13.90 \\
\hline AUG 31 & 4.89 & 20 & 11.99 & MAR 02, 1981 & 16.25 \\
\hline MAR 02, 1984 & 10.22 & 25 & 12.20 & SEP 17 & 21.66 \\
\hline SEP 07 & 4.12 & 30 & 12.28 & MAR 08, 1982 & 19.93 \\
\hline MAR 04, 1985 & 10.52 & DEC 05 & 12.49 & SEP 08 & 10.63 \\
\hline SEP 19 & 7.52 & 10 & 12.57 & MAR 02, 1983 & 12.85 \\
\hline MAR 10, 1986 & 6.64 & 15 & 12.71 & AUG 31 & 6.28 \\
\hline SEP 29 & 7.76 & 20 & 12.81 & MAR 02, 1984 & 10.21 \\
\hline MAR 02, 1987 & 10.14 & 25 & 12.91 & SEP 05 & 4.60 \\
\hline SEP 15 & 9.59 & 31 & 13.30 & MAR 04, 1985 & 10.07 \\
\hline MAR 03, 1988 & 10.40 & JAN 05, 1970 & 13.59 & SEP 19 & 12.51 \\
\hline SEP 26 & 9.25 & 10 & 13.57 & MAR 10, 1986 & 15.58 \\
\hline MAR 08, 1989 & 10.25 & 15 & 13.75 & SEP 29 & 7.72 \\
\hline SEP 19 & 8.09 & 20 & 13.97 & MAR 03, 1987 & 14.08 \\
\hline
\end{tabular}


Table 3.-Water levels in selected wells-Continued

\begin{tabular}{|c|c|c|c|c|c|}
\hline Date & Water level & Date & Water level & Date & Water level \\
\hline \multicolumn{2}{|c|}{ (A-11-1)4daa-2-Continued } & \multicolumn{2}{|c|}{ (A-11-1)18baa-1-Continued } & \multicolumn{2}{|c|}{ (A-11-1)18ddd-1-Continued } \\
\hline SEP 15,1987 & 18.19 & MAR 02, 1984 & -37.3 & MAR 12, 1979 & -3.9 \\
\hline MAR 03, 1988 & 21.01 & SEP 07 & -41.0 & SEP 17 & -3.6 \\
\hline SEP 26 & 25.35 & MAR 04, 1985 & -37.8 & MAR 10, 1980 & -2.1 \\
\hline MAR 08, 1989 & 23.36 & SEP 19 & -36.2 & SEP 17 & -4.8 \\
\hline SEP 19 & 21.17 & MAR 10, 1986 & -37.2 & MAR 17, 1981 & -2.6 \\
\hline MAR 01, 1990 & 21.88 & SEP 29 & -38.5 & MAR 08, 1982 & -2.0 \\
\hline APR 27 & 21.66 & MAR 05, 1987 & -35.3 & SEP 08 & -8.4 \\
\hline MAY 29 & 21.97 & SEP 15 & -33.2 & MAR 02, 1983 & -7.2 \\
\hline JUN 26 & 24.52 & MAR 03, 1988 & -32.3 & AUG 31 & -10.8 \\
\hline JUL 25 & 25.10 & SEP 26 & -30.0 & MAR 02, 1984 & -7.6 \\
\hline AUG 20 & 26.44 & MAR 15, 1989 & -30.8 & SEP 07 & -11.8 \\
\hline 25 & 27.30 & SEP 19 & -31.3 & MAR 04, 1985 & -7.7 \\
\hline 28 & 27.70 & MAR 01, 1990 & -30.9 & SEP 19 & -5.6 \\
\hline 31 & 27.73 & APR 27 & .32 .1 & MAR 10, 1986 & -4.0 \\
\hline SEP 05 & 27.78 & MAY 30 & -29.9 & SEP 29 & -10.1 \\
\hline 10 & 27.73 & JUN 28 & -29.0 & MAR 05, 1987 & -4.8 \\
\hline 15 & 28.17 & JUL 26 & -28.0 & & \\
\hline 20 & 27.25 & AUG 27 & -27.3 & $(A-11-1) 21 d d b-1$ & \\
\hline 25 & 27.33 & SEP 27 & -28.7 & Altitude 4,550 & \\
\hline 27 & 28.17 & OCT 29 & -30.0 & SEP 08, 1982 & 4.26 \\
\hline 30 & 26.57 & NOV 28 & -30.7 & MAR 01, 1983 & 5.00 \\
\hline OCT 05 & 26.30 & JAN 17, 1991 & -30.3 & AUG 31 & 4.50 \\
\hline 10 & 25.35 & MAR 08 & -30.2 & MAR 02,1984 & 5.32 \\
\hline 15 & 25.04 & & & SEP 07 & 4.18 \\
\hline 20 & 24.60 & (A-11-1)18ddd-1 & & MAR 04, 1985 & 5.40 \\
\hline 25 & 24.29 & Altitude 4,480 & & SEP 19 & 4.52 \\
\hline 31 & 24.44 & AUG 04, 1969 & -5.7 & MAR 10, 1986 & 4.86 \\
\hline NOV 05 & 23.76 & SEP 04 & -6.2 & SEP 29 & 4.12 \\
\hline 06 & 24.19 & OCT 09 & -7.3 & MAR 03, 1987 & 5.49 \\
\hline 10 & 23.83 & NOV 07 & -7.8 & SEP 15 & 4.67 \\
\hline 15 & 23.79 & DEC 02 & -7.0 & MAR 03, 1988 & 5.51 \\
\hline 20 & 23.41 & JAN 07, 1970 & -6.4 & SEP 26 & 5.08 \\
\hline 25 & 23.40 & FEB 09 & -5.5 & MAR 08, 1989 & 4.65 \\
\hline 27 & 23.74 & MAR 04 & -5.0 & SEP 19 & 4.43 \\
\hline 30 & 23.52 & SEP 02 & -6.1 & MAR 01, 1990 & 5.17 \\
\hline DEC 05 & 23.58 & MAR 01, 1971 & -5.2 & APR 27 & 5.16 \\
\hline 10 & 23.60 & SEP 01 & -8.9 & MAY 29 & 4.37 \\
\hline 15 & 23.57 & MAR 01, 1972 & -6.8 & JUN 26 & 4.79 \\
\hline 20 & 23.59 & SEP 12 & -9.3 & JUL 26 & 4.88 \\
\hline 25 & 24.00 & MAR 01, 1973 & -6.3 & AUG 28 & 5.28 \\
\hline 31 & 24.19 & SEP 13 & -7.2 & SEP 27 & 4.70 \\
\hline JAN 03, 1991 & 24.67 & MAR 13,1974 & -7.2 & OCT 29 & 5.24 \\
\hline 05 & 24.19 & SEP 11 & -8.9 & NOV 28 & 5.45 \\
\hline 10 & 24.15 & MAR 12,1975 & -6.6 & JAN 03, 1991 & 6.00 \\
\hline 15 & 24.15 & OCT 15 & -9.4 & MAR 08 & 5.25 \\
\hline MAR 08 & 24.75 & MAR 15, 1976 & -6.7 & & \\
\hline & & SEP 13 & -9.2 & $(A-11-1) 27 c d c-1$ & \\
\hline$(A-11-1) 18 b a a-1$ & & MAR 08, 1977 & -5.7 & Altitude 4,600 & \\
\hline Altitude 4,440 & & SEP 28 & -2.0 & AUG 05, 1969 & 116.23 \\
\hline MAR 02, 1983 & -37.2 & MAR 08, 1978 & -2.5 & SEP 04 & 116.12 \\
\hline AUG 31 & -40.5 & SEP 19 & -2.1 & OCT 09 & 115.13 \\
\hline
\end{tabular}


Table 3.-Water levels in selected wells-Continued

\begin{tabular}{|c|c|c|c|c|c|}
\hline Date & Water level & Date & Water level & Date & Water level \\
\hline \multicolumn{2}{|c|}{ (A-11-1)27cdc-1-Continued } & \multicolumn{2}{|c|}{ (A-11-1)27cdc-1-Continued } & \multicolumn{2}{|c|}{ (A-12-1)3bbb-1-Continued } \\
\hline NOV 07,1969 & 114.99 & NOV 28,1990 & 126.26 & SEP 18, 1989 & 16.46 \\
\hline DEC 02 & 115.76 & JAN 03, 1991 & 126.38 & MAR 01, 1990 & 21.28 \\
\hline JAN 07, 1970 & 116.95 & MAR 08 & 126.78 & APR 26 & 22.23 \\
\hline FEB 09 & 117.93 & & & MAY 29 & 21.13 \\
\hline MAR 04 & 119.50 & $(A-12-1) 3 b b b-1$ & & JUN 26 & 19.73 \\
\hline SEP 02 & 117.77 & Altitude 4,510 & & JUL 25 & 23.36 \\
\hline MAR 15, 1971 & 118.99 & AUG 04, 1969 & 3.85 & AUG 27 & 26.08 \\
\hline SEP 01 & 116.04 & SEP 04 & 6.90 & SEP 26 & 25.76 \\
\hline MAR 01, 1972 & 116.29 & OCT 08 & 5.27 & OCT 31 & 23.94 \\
\hline SEP 12 & 111.94 & NOV 06 & 6.84 & NOV 27 & 23.69 \\
\hline MAR 02, 1973 & 116.70 & DEC 03 & 9.29 & JAN 03, 1991 & 24.34 \\
\hline SEP 13 & 114.25 & JAN 06, 1970 & 12.15 & MAR 08 & 25.37 \\
\hline MAR 13, 1974 & 117.80 & FEB 10 & 14.30 & & \\
\hline SEP 11 & 112.36 & MAR 03 & 15.63 & $(A-12-1) 17$ daa-1 & \\
\hline MAR 11, 1975 & 116.09 & SEP 02 & 8.30 & Altitude 4,450 & \\
\hline OCT 15 & 111.78 & MAR 01, 1971 & 14.48 & APR 26, 1990 & -13.5 \\
\hline MAR 15, 1976 & 116.10 & SEP 01 & .77 & MAY 29 & -13.3 \\
\hline SEP 13 & 111.89 & MAR 01, 1972 & 13.79 & JUN 28 & -13.2 \\
\hline MAR 08, 1977 & 117.19 & 01 & 15.50 & JUL 25 & -12.9 \\
\hline SEP 28 & 122.01 & SEP 12 & 4.67 & AUG 28 & -12.2 \\
\hline MAR 08, 1978 & 121.87 & MAR 14,1974 & 16.10 & SEP 26 & -12.4 \\
\hline SEP 19 & 118.55 & SEP 13 & 2.63 & OCT 31 & -13.5 \\
\hline MAR 12, 1979 & 120.11 & MAR 10,1975 & 14.98 & NOV 30 & -13.7 \\
\hline SEP 17 & 119.02 & OCT 14 & 2.31 & JAN 17, 1991 & -13.7 \\
\hline MAR 10,1980 & 120.70 & MAR 16, 1976 & 15.10 & MAR 09 & -13.5 \\
\hline SEP 17 & 117.23 & SEP 20 & 2.93 & & \\
\hline MAR 02, 1981 & 119.79 & MAR 11,1977 & 16.36 & (A-12-1)24add-1 & \\
\hline SEP 17 & 137.82 & OCT 12 & 18.28 & Altitude 5,060 & \\
\hline MAR 08, 1982 & 123.32 & MAR 09, 1978 & 21.75 & JAN 04, 1991 & 65.22 \\
\hline SEP 08 & 111.80 & SEP 20 & 7.05 & 18 & 65.36 \\
\hline MAR 02, 1983 & 114.36 & MAR 13, 1979 & 18.23 & APR 02, 1991 & 64.57 \\
\hline AUG 31 & 118.20 & SEP $20^{\circ}$ & 12.75 & & \\
\hline MAR 02, 1984 & 116.78 & MAR 11,1980 & 18.87 & $(A-12-1) 29 c a b-1$ & \\
\hline SEP 07 & 107.40 & SEP 18 & 5.37 & Altitude $\mathbf{4 , 4 4 0}$ & \\
\hline MAR 04, 1985 & 113.54 & MAR 04, 1981 & 17.73 & AUG 25, 1969 & -17.5 \\
\hline SEP 19 & 120.64 & SEP 22 & 20.40 & 31 & -17.4 \\
\hline MAR 14, 1986 & 120.40 & MAR 08, 1982 & 20.35 & SEP 05 & -17.8 \\
\hline SEP 30 & 112.08 & SEP 08 & 5.24 & 10 & -18.0 \\
\hline MAR 03, 1987 & 117.74 & MAR 03,1983 & 18.40 & 15 & -18.2 \\
\hline SEP 15 & 120.63 & SEP 02 & 5.54 & 20 & -18.5 \\
\hline MAR 03, 1988 & 123.65 & MAR 05, 1984 & 16.66 & 25 & -18.6 \\
\hline SEP 26 & 130.80 & SEP 11 & 3.83 & 30 & -18.7 \\
\hline MAR 08, 1989 & 124.98 & MAR 05, 1985 & 16.76 & ОСТ 05 & -18.9 \\
\hline SEP 19 & 124.63 & SEP 05 & 4.77 & 10 & -19.0 \\
\hline MAR 01, 1990 & 124.72 & MAR 11,1986 & 15.50 & 15 & -19.0 \\
\hline MAY 29 & 125.62 & SEP 29 & 2.51 & 20 & -19.0 \\
\hline JUN 26 & 126.20 & MAR 04, 1987 & 15.35 & 25 & -19.0 \\
\hline JUL 26 & 128.90 & SEP 15 & 14.41 & 31 & -18.9 \\
\hline AUG 28 & 129.69 & MAR 04, 1988 & 20.18 & NOV 05 & -19.6 \\
\hline SEP 27 & 129.18 & SEP 26 & 26.68 & 10 & -19.7 \\
\hline OCT 30 & 126.70 & MAR 15, 1989 & 23.30 & 15 & -19.5 \\
\hline
\end{tabular}


Table 3.-Water levels in selected wells-Continued

\begin{tabular}{|c|c|c|c|c|c|}
\hline Date & Water level & Date & Water level & Date & Water level \\
\hline \multicolumn{2}{|c|}{ (A-12-1)29cab-1-Continued } & \multicolumn{2}{|c|}{ (A-12-1)29cab-1-Continued } & \multicolumn{2}{|c|}{ (A-12-1)29cab-1-Continued } \\
\hline NOV 20, 1969 & -19.5 & AUG 05, 1970 & -18.2 & AUG 05,1971 & -19.3 \\
\hline 25 & -19.5 & 10 & -18.3 & 10 & -19.2 \\
\hline 30 & -19.4 & 15 & -18.3 & 15 & -19.1 \\
\hline DEC 05 & -19.4 & 20 & -18.5 & 20 & -19.9 \\
\hline 10 & -19.4 & 25 & -17.6 & 25 & -20.2 \\
\hline 15 & -19.3 & 31 & -17.6 & 31 & -20.5 \\
\hline 20 & -19.0 & SEP 05 & -17.8 & SEP 05 & -20.5 \\
\hline 25 & -19.0 & 10 & -18.6 & 10 & -20.2 \\
\hline 31 & -18.9 & 15 & -18.7 & 15 & -21.0 \\
\hline JAN 05, 1970 & -18.9 & 20 & -18.7 & 20 & -20.8 \\
\hline 10 & -18.8 & 25 & -18.6 & 25 & -20.8 \\
\hline 15 & -18.8 & 30 & -19.1 & 30 & -20.8 \\
\hline 20 & -18.8 & OCT 05 & .19 .2 & ОСТ 05 & -20.5 \\
\hline 25 & -18.8 & 10 & -18.7 & 10 & -20.5 \\
\hline 31 & -18.8 & 15 & -18.8 & 15 & -20.8 \\
\hline FEB 05 & -18.7 & 20 & -18.5 & 20 & -20.7 \\
\hline 10 & -18.5 & 25 & -18.3 & 25 & -20.5 \\
\hline 15 & -18.4 & 31 & -18.6 & 31 & -20.4 \\
\hline 20 & -18.6 & NOV 05 & -18.7 & NOV 05 & -20.4 \\
\hline 25 & -18.5 & 10 & -18.5 & 10 & -20.5 \\
\hline 28 & -18.5 & 15 & -18.5 & 15 & -19.8 \\
\hline MAR 05 & -18.4 & 20 & -18.7 & 20 & -19.8 \\
\hline 10 & -18.4 & 25 & -18.7 & 25 & -19.8 \\
\hline 15 & -18.4 & 30 & -18.5 & 30 & -19.8 \\
\hline 20 & -18.3 & DEC 05 & -18.3 & DEC 05 & -19.7 \\
\hline 25 & -18.2 & 10 & -18.2 & 10 & -19.7 \\
\hline 31 & -18.2 & 15 & -18.0 & 15 & -19.7 \\
\hline APR 05 & -18.2 & 20 & -17.9 & 20 & -19.5 \\
\hline 10 & -18.2 & 25 & -17.9 & 25 & -19.8 \\
\hline 15 & -18.0 & 31 & -18.2 & 31 & -19.5 \\
\hline 20 & -18.0 & JAN 31, 1971 & -18.0 & JAN 05, 1972 & -19.2 \\
\hline 25 & -18.0 & FEB 05 & -17.9 & 10 & -19.5 \\
\hline 30 & -18.0 & 10 & -17.9 & 15 & -19.3 \\
\hline MAY 05 & -17.9 & 15 & -17.8 & 20 & -19.3 \\
\hline 10 & -17.8 & 20 & -17.7 & 25 & -19.5 \\
\hline 15 & -18.2 & 25 & -17.6 & 31 & -19.3 \\
\hline 20 & -18.3 & 28 & -17.6 & FEB 05 & -19.2 \\
\hline 25 & -18.0 & MAR 05 & -18.0 & 10 & -19.0 \\
\hline 31 & -17.7 & 10 & -18.0 & 15 & -19.1 \\
\hline JUN 05 & -18.0 & 15 & -17.9 & 20 & -18.8 \\
\hline 10 & -17.3 & 20 & -17.8 & 25 & -19.0 \\
\hline 15 & -17.0 & 25 & -17.8 & 29 & -18.8 \\
\hline 20 & -17.1 & 31 & -17.8 & MAR 05 & -18.7 \\
\hline 25 & -17.1 & APR 25 & -18.4 & 10 & -19.1 \\
\hline 30 & -17.5 & 30 & -18.5 & 15 & -18.9 \\
\hline JUL 05 & -17.1 & MAY 05 & -18.1 & 20 & -18.7 \\
\hline 10 & -17.0 & 31 & -18.5 & 25 & -19.0 \\
\hline 15 & -17.2 & JUN 30 & -18.6 & 31 & -19.0 \\
\hline 20 & -17.3 & JUL 20 & -19.5 & APR 05 & -18.8 \\
\hline 25 & -17.6 & 25 & -19.9 & 10 & -18.8 \\
\hline 31 & -18.3 & 31 & -18.7 & 15 & -18.8 \\
\hline
\end{tabular}


Table 3.-Water levels in selected wells-Continued

\begin{tabular}{|c|c|c|c|c|c|}
\hline Date & Water level & Date & Water level & Date & Water level \\
\hline \multicolumn{2}{|c|}{ (A-12-1)29cab-1-Continued } & \multicolumn{2}{|c|}{$(A-12-1) 29 c a b-1$-Continued } & \multicolumn{2}{|c|}{ (A-12-1)29cab-1-Continued } \\
\hline APR 20, 1972 & -18.6 & JAN 05, 1973 & -19.6 & SEP 20,1973 & -19.8 \\
\hline 25 & -18.8 & 10 & -19.5 & 25 & -19.0 \\
\hline 30 & -18.7 & 15 & -19.5 & 30 & -18.9 \\
\hline MAY 05 & -19.0 & 20 & -19.5 & OCT 05 & -19.4 \\
\hline 10 & -18.8 & 25 & -19.5 & 10 & -19.0 \\
\hline 15 & -18.6 & 31 & -19.3 & 15 & -19.2 \\
\hline 20 & -18.4 & FEB 05 & -19.2 & 20 & -19.2 \\
\hline 25 & -18.5 & 10 & -19.1 & 25 & -19.1 \\
\hline 31 & -18.9 & 15 & -18.8 & 31 & -19.1 \\
\hline JUN 05 & -18.8 & 20 & -18.8 & NOV 05 & -18.9 \\
\hline 10 & -19.0 & 25 & -18.8 & 10 & -18.7 \\
\hline 15 & -19.1 & 28 & -18.8 & 15 & -18.5 \\
\hline 20 & -19.2 & MAR 05 & -18.8 & 20 & -18.2 \\
\hline 25 & -19.5 & 10 & -18.0 & 25 & -17.9 \\
\hline 30 & -19.9 & 15 & -17.7 & 30 & -18.0 \\
\hline JUL 05 & -19.5 & 20 & -17.7 & DEC 05 & -18.1 \\
\hline 10 & -19.2 & 25 & -17.6 & 10 & -18.4 \\
\hline 15 & -19.3 & 31 & -17.4 & 15 & -18.0 \\
\hline 20 & -19.8 & APR 05 & -18.0 & 20 & -18.2 \\
\hline 25 & -19.7 & 10 & -17.9 & 25 & -18.2 \\
\hline 31 & -19.8 & 15 & -17.6 & 31 & -18.0 \\
\hline AUG 05 & -19.9 & 20 & -17.4 & JAN 05, 1974 & -18.0 \\
\hline 10 & -20.0 & 25 & -17.9 & 10 & -18.0 \\
\hline 15 & -20.1 & 30 & -17.5 & 15 & -18.0 \\
\hline 20 & -20.0 & MAY 05 & -17.8 & 20 & -18.0 \\
\hline 25 & -20.5 & 10 & -18.0 & 25 & -18.0 \\
\hline 31 & -20.5 & 15 & -17.7 & 31 & -18.0 \\
\hline SEP 05 & -20.2 & 20 & -17.0 & FEB 05 & -18.0 \\
\hline 10 & -20.7 & 25 & -17.3 & 10 & -17.9 \\
\hline 15 & -21.0 & 31 & -17.8 & 15 & -17.8 \\
\hline 20 & -21.0 & JUN 05 & -17.9 & 20 & -17.5 \\
\hline 25 & -20.9 & 10 & -17.5 & 25 & -17.6 \\
\hline 30 & -20.6 & 15 & -17.5 & 28 & -17.5 \\
\hline OCT 05 & -20.7 & 20 & -17.5 & MAR 05 & -17.5 \\
\hline 10 & -20.8 & 25 & -17.6 & 10 & -17.5 \\
\hline 15 & -20.5 & 30 & -17.6 & 15 & -17.4 \\
\hline 20 & -20.6 & JUL 05 & -17.7 & 20 & -17.6 \\
\hline 25 & -20.7 & 10 & -17.8 & 25 & -17.6 \\
\hline 31 & -20.8 & 15 & -17.9 & 31 & -17.5 \\
\hline NOV 05 & -20.8 & 20 & -18.0 & APR 05 & -17.6 \\
\hline 10 & -20.8 & 25 & -18.2 & 10 & -17.5 \\
\hline 15 & -20.8 & 31 & -18.5 & 15 & -17.5 \\
\hline 20 & -20.8 & AUG 05 & -18.0 & 20 & -17.8 \\
\hline 25 & -20.8 & 10 & -18.5 & 25 & -18.0 \\
\hline 30 & -20.8 & 15 & -18.4 & 30 & -18.0 \\
\hline DEC 05 & -20.4 & 20 & -18.0 & MAY 05 & -17.7 \\
\hline 10 & -20.0 & 25 & -18.3 & 10 & -16.7 \\
\hline 15 & -19.8 & 31 & -18.7 & 15 & -17.2 \\
\hline 20 & -19.8 & SEP 05 & -18.9 & 20 & -17.1 \\
\hline 25 & -19.8 & 10 & -19.1 & 25 & -17.3 \\
\hline 31 & -19.8 & 15 & -19.5 & 31 & -17.8 \\
\hline
\end{tabular}


Table 3.-Water levels in selected wells-Continued

\begin{tabular}{|c|c|c|c|c|c|}
\hline Date & Water level & Date & Water level & & Water level \\
\hline \multicolumn{2}{|c|}{ (A-12-1)29cab-1-Continued } & \multicolumn{2}{|c|}{ (A-12-1)29cab-1-Continued } & \multicolumn{2}{|c|}{ (A-12-1)29cab-1-Continued } \\
\hline JUN 05, 1974 & -18.0 & FEB 20, 1975 & -19.3 & NOV 05,1975 & -21.0 \\
\hline 10 & -18.0 & 25 & -18.9 & 10 & -21.0 \\
\hline 15 & -18.0 & 28 & -18.8 & 15 & -21.0 \\
\hline 20 & -18.0 & MAR 05 & -18.8 & 20 & -21.0 \\
\hline 25 & -18.0 & 10 & -18.5 & 25 & -20.9 \\
\hline 30 & -18.0 & 15 & -18.4 & 30 & -20.9 \\
\hline JUL 05 & -19.0 & 20 & -18.5 & DEC 05 & -20.8 \\
\hline 10 & -18.5 & 25 & -18.6 & 10 & -20.4 \\
\hline 15 & -18.0 & 31 & -18.0 & 15 & -20.5 \\
\hline 20 & -18.5 & APR 05 & -18.2 & 20 & -20.3 \\
\hline 25 & -18.8 & 10 & -18.1 & 25 & -20.4 \\
\hline 31 & -19.2 & 15 & -18.1 & 31 & -20.7 \\
\hline AUG 05 & -19.0 & 20 & -17.9 & JAN 05, 1976 & -20.5 \\
\hline 10 & -19.0 & 25 & -21.7 & 10 & -20.2 \\
\hline 15 & -20.0 & 30 & -18.3 & 15 & -20.2 \\
\hline 20 & -19.5 & MAY 05 & -18.0 & 20 & -20.5 \\
\hline 25 & -18.5 & 10 & -18.0 & 25 & -18.5 \\
\hline 31 & -18.7 & 15 & -18.6 & 31 & -18.5 \\
\hline SEP 05 & -19.6 & 20 & -18.4 & FEB 05 & -19.8 \\
\hline 10 & -19.4 & 25 & -18.0 & 10 & -19.5 \\
\hline 15 & -19.5 & 31 & -18.0 & 15 & -19.3 \\
\hline 20 & -19.6 & JUN 05 & -18.5 & 20 & -19.4 \\
\hline 25 & -20.0 & 10 & -18.8 & 25 & -19.8 \\
\hline 30 & -19.9 & 15 & -18.3 & 29 & -19.9 \\
\hline OCT 05 & -19.8 & 20 & -18.7 & MAR 05 & -19.6 \\
\hline 10 & -20.0 & 25 & -19.0 & 10 & -19.7 \\
\hline 15 & -20.2 & 30 & -19.0 & 15 & -19.3 \\
\hline 20 & -20.3 & JUL 05 & -18.7 & 20 & -19.0 \\
\hline 25 & -20.2 & 10 & -18.5 & 25 & -19.0 \\
\hline 31 & -20.0 & 15 & -19.0 & 31 & -19.1 \\
\hline NOV 05 & -19.9 & 20 & -18.3 & APR 05 & -19.0 \\
\hline 10 & -20.0 & 25 & -19.0 & 10 & -19.0 \\
\hline 15 & -20.1 & 31 & -19.7 & 15 & -19.0 \\
\hline 20 & -20.2 & AUG 05 & -19.2 & 20 & -19.0 \\
\hline 25 & -20.2 & 10 & -19.0 & 23 & -19.0 \\
\hline 30 & -19.9 & 15 & -19.5 & 25 & -19.0 \\
\hline DEC 05 & -19.7 & 20 & -19.3 & 30 & -19.2 \\
\hline 10 & -19.6 & 25 & -19.4 & MAY 05 & -19.2 \\
\hline 15 & -19.2 & 31 & -19.5 & 10 & -19.0 \\
\hline 20 & -19.6 & SEP 05 & -19.8 & 15 & -18.5 \\
\hline 25 & -19.7 & 10 & -19.3 & 20 & -18.7 \\
\hline 31 & -19.3 & 15 & -20.2 & 25 & -18.9 \\
\hline JAN 05, 1975 & -19.0 & 20 & -20.3 & 31 & -18.5 \\
\hline 10 & -19.1 & 25 & -20.5 & JUN 05 & -18.5 \\
\hline 15 & -19.3 & 30 & -20.5 & 10 & -18.5 \\
\hline 20 & -19.2 & OCT 05 & -20.5 & 15 & -19.4 \\
\hline 25 & -19.1 & 10 & -20.7 & 20 & -19.5 \\
\hline 31 & -19.2 & 15 & -20.8 & 25 & -19.4 \\
\hline FEB 05 & -19.6 & 20 & -21.0 & 30 & -19.0 \\
\hline 10 & -19.4 & 25 & -20.7 & JUL 05 & -18.5 \\
\hline 15 & -19.2 & 31 & -20.9 & 10 & -18.8 \\
\hline
\end{tabular}


Table 3.-Water levels in selected wells-Continued

\begin{tabular}{|c|c|c|c|c|c|}
\hline Date & Water level & Date & Water level & Date & Water level \\
\hline \multicolumn{2}{|c|}{ (A-12-1)29cab-1-Continued } & \multicolumn{2}{|c|}{ (A-12-1)29cab-1-Continued } & \multicolumn{2}{|c|}{ (A-12-1)29cab-1-Continued } \\
\hline JUL 15, 1976 & -19.4 & MAR 31, 1977 & -19.0 & DEC 15,1977 & -17.6 \\
\hline 20 & -19.6 & APR 05 & -18.5 & 20 & -17.5 \\
\hline 25 & -19.2 & 10 & -18.2 & 25 & -17.5 \\
\hline 31 & -19.5 & 15 & -18.2 & 31 & -17.5 \\
\hline AUG 05 & -20.2 & 20 & -18.6 & JAN 05, 1978 & -18.3 \\
\hline 10 & -20.1 & 25 & -18.1 & 10 & -17.5 \\
\hline 15 & -20.0 & 30 & -18.2 & 15 & -17.3 \\
\hline 20 & -20.6 & MAY 05 & -18.5 & 20 & -17.5 \\
\hline 25 & -20.5 & 10 & -17.9 & 25 & -17.4 \\
\hline 31 & -20.5 & 15 & -17.8 & 31 & -17.4 \\
\hline SEP 05 & -20.5 & 20 & -18.1 & FEB 05 & -18.3 \\
\hline 10 & -20.8 & 25 & -18.2 & 10 & -17.4 \\
\hline 15 & -21.0 & 31 & -18.8 & 15 & -17.5 \\
\hline 20 & -20.9 & JUN 05 & -18.0 & 20 & -17.3 \\
\hline 25 & -20.6 & 10 & -18.0 & 25 & -17.2 \\
\hline 30 & -21.0 & 15 & -18.0 & 28 & -17.4 \\
\hline ОСТ 05 & -21.0 & 20 & -17.5 & MAR 05 & -17.0 \\
\hline 10 & -21.0 & 25 & -16.6 & 10 & -17.0 \\
\hline 15 & -21.1 & 30 & -16.5 & 15 & -17.5 \\
\hline 20 & -21.0 & JUL 05 & -16.5 & 20 & -17.2 \\
\hline 25 & -21.0 & 10 & -15.9 & 25 & -17.2 \\
\hline 31 & -20.8 & 15 & -15.8 & 31 & -17.5 \\
\hline NOV 05 & -20.9 & 20 & -15.5 & APR 05 & -17.3 \\
\hline 10 & -21.0 & 25 & -15.6 & 10 & -17.3 \\
\hline 15 & -20.7 & 31 & -15.5 & 15 & -17.4 \\
\hline 20 & -20.5 & AUG 05 & -15.3 & 20 & -17.5 \\
\hline 25 & -20.7 & 10 & -15.2 & 25 & -17.5 \\
\hline 30 & -20.5 & 15 & -15.0 & 30 & -17.1 \\
\hline DEC 05 & -20.3 & 20 & -16.3 & MAY 05 & -17.4 \\
\hline 10 & -20.3 & 25 & -16.7 & 10 & -17.5 \\
\hline 15 & -20.3 & 31 & -17.4 & 15 & -17.4 \\
\hline 20 & -20.4 & SEP 05 & -17.0 & 20 & -17.1 \\
\hline 25 & -20.2 & 10 & -17.0 & 25 & -17.2 \\
\hline 31 & -20.1 & 15 & -17.0 & 31 & -17.3 \\
\hline JAN 05, 1977 & -19.5 & 20 & -17.4 & JUN 05 & -17.1 \\
\hline 10 & -19.6 & 25 & -17.7 & 10 & -16.8 \\
\hline 15 & -19.1 & 30 & -17.5 & 15 & -16.8 \\
\hline 20 & -19.1 & ОСТ 05 & -18.0 & 20 & -17.0 \\
\hline 25 & -19.7 & 10 & -17.8 & 25 & -16.8 \\
\hline 31 & -20.3 & 15 & -17.7 & 30 & -16.6 \\
\hline FEB 05 & -19.7 & 20 & -17.7 & JUL 05 & -16.5 \\
\hline 10 & -19.7 & 25 & -17.9 & 10 & -17.0 \\
\hline 15 & -19.3 & 31 & -17.6 & 15 & -17.0 \\
\hline 20 & -19.4 & NOV 05 & -17.7 & 20 & -17.0 \\
\hline 25 & -19.3 & 10 & -17.9 & 25 & -16.8 \\
\hline 28 & -19.3 & 15 & -17.9 & 31 & -17.3 \\
\hline MAR 05 & -19.3 & 20 & -17.5 & AUG 05 & -16.7 \\
\hline 10 & -19.0 & 25 & -17.7 & 10 & -16.8 \\
\hline 15 & -19.0 & 30 & -17.9 & 15 & -18.0 \\
\hline 20 & -18.9 & DEC 05 & -17.5 & 20 & -18.4 \\
\hline 25 & -18.4 & 10 & -17.4 & 25 & -18.0 \\
\hline
\end{tabular}


Table 3.-Water levels in selected wells-Continued

\begin{tabular}{|c|c|c|c|c|c|}
\hline Date & Water level & Date & Water level & Date & Water level \\
\hline \multicolumn{2}{|c|}{ (A-12-1)29cab-1-Continued } & \multicolumn{2}{|c|}{ (A-12-1)29cab-1-Continued } & \multicolumn{2}{|c|}{ (A-12-1)29cab-1-Continued } \\
\hline AUG 31, 1978 & -17.4 & MAY 15,1979 & -18.5 & JAN 31, 1980 & -19.0 \\
\hline SEP 05 & -17.3 & 20 & -17.0 & FEB 05 & -18.9 \\
\hline 10 & -17.5 & 25 & -17.5 & 10 & -19.0 \\
\hline 15 & -18.5 & 31 & -17.5 & 15 & -18.1 \\
\hline 20 & -18.5 & JUN 05 & -17.3 & 20 & -18.7 \\
\hline 25 & -19.0 & 10 & -17.5 & 25 & -18.4 \\
\hline 30 & -19.0 & 15 & -17.0 & 29 & -18.4 \\
\hline OCT 05 & -19.3 & 20 & -17.6 & MAR 05 & -18.3 \\
\hline 10 & -19.4 & 25 & -17.6 & 10 & -18.5 \\
\hline 15 & -19.4 & 30 & -17.4 & 15 & -18.3 \\
\hline 20 & -19.3 & JUL 05 & -16.9 & 20 & -18.3 \\
\hline 25 & -19.5 & 10 & -16.8 & 25 & -18.0 \\
\hline 31 & -19.5 & 15 & -17.7 & 31 & -18.2 \\
\hline NOV 05 & -19.3 & 20 & -18.0 & APR 05 & -18.1 \\
\hline 10 & -19.3 & 25 & -18.1 & 10 & -18.1 \\
\hline 15 & -20.0 & 31 & -17.9 & 15 & -18.6 \\
\hline 20 & -19.6 & AUG 05 & -18.3 & 20 & -18.6 \\
\hline 25 & -19.5 & 10 & -18.0 & 25 & -18.3 \\
\hline 30 & -19.0 & 15 & -17.8 & 30 & -18.2 \\
\hline DEC 05 & -19.2 & 20 & -18.0 & MAY 05 & -18.3 \\
\hline 10 & -19.6 & 25 & -17.6 & 10 & -17.9 \\
\hline 15 & -19.2 & 31 & -18.0 & 15 & -18.5 \\
\hline 20 & -19.0 & SEP 05 & -18.7 & 20 & -18.4 \\
\hline 25 & -19.3 & 10 & -18.2 & 25 & -18.4 \\
\hline 31 & -19.0 & 15 & -18.4 & 31 & -18.6 \\
\hline JAN 05, 1979 & -18.9 & 20 & -18.4 & JUN 05 & -18.9 \\
\hline 10 & -18.6 & 25 & -18.5 & 10 & -18.8 \\
\hline 15 & -18.5 & 30 & -19.0 & 15 & -18.2 \\
\hline 20 & -18.5 & OCT 05 & -19.4 & 20 & -18.0 \\
\hline 25 & -18.5 & 10 & -19.4 & 25 & -18.0 \\
\hline 31 & -18.5 & 15 & -19.3 & 30 & -18.0 \\
\hline FEB 05 & -18.8 & 20 & -19.2 & JUL 05 & -18.4 \\
\hline 10 & -18.5 & 25 & -19.5 & 10 & -18.5 \\
\hline 15 & -18.5 & 31 & -19.5 & 15 & -18.4 \\
\hline 20 & -18.5 & NOV 05 & -19.3 & 20 & -19.4 \\
\hline 25 & -18.4 & 10 & -19.6 & 25 & -18.0 \\
\hline 28 & -18.5 & 15 & -19.6 & 31 & -17.3 \\
\hline MAR 05 & -18.5 & 20 & -19.3 & AUG 05 & -18.5 \\
\hline 10 & -18.5 & 25 & -19.3 & 10 & -18.5 \\
\hline 15 & -18.4 & 30 & -19.4 & 15 & -18.3 \\
\hline 20 & -18.5 & DEC 05 & -19.2 & 20 & -19.6 \\
\hline 25 & -18.3 & 10 & -19.2 & 25 & -19.4 \\
\hline 31 & -18.1 & 15 & -19.3 & 31 & -19.7 \\
\hline APR 05 & -18.3 & 20 & -19.5 & SEP 05 & -19.7 \\
\hline 10 & -18.0 & 25 & -19.0 & 10 & -19.6 \\
\hline 15 & -18.2 & 31 & -18.7 & 15 & -20.3 \\
\hline 20 & -18.5 & JAN 05, 1980 & -19.2 & 20 & -20.3 \\
\hline 25 & -18.0 & 10 & -19.0 & 25 & -20.4 \\
\hline 30 & -18.0 & 15 & -19.0 & 30 & -20.5 \\
\hline MAY 05 & -18.0 & 20 & -18.9 & OCT 05 & -20.5 \\
\hline 10 & -18.0 & 25 & -19.2 & 10 & -20.7 \\
\hline
\end{tabular}


Table 3.-Water levels in selected wells-Continued

\begin{tabular}{|c|c|c|c|c|c|}
\hline Date & Water level & Date & Water level & Date & Water level \\
\hline \multicolumn{2}{|c|}{ (A-12-1)29cab-1-Continued } & \multicolumn{2}{|c|}{ (A-12-1)29cab-1-Continued } & \multicolumn{2}{|c|}{ (A-12-1)29cab-1-Continued } \\
\hline OCT 15,1980 & -20.5 & JUN 30, 1981 & -18.6 & MAR 15, 1982 & -17.2 \\
\hline 20 & -20.4 & JUL 05 & -17.5 & 20 & -17.1 \\
\hline 25 & -20.3 & 10 & -17.7 & 25 & -17.4 \\
\hline 31 & -20.4 & 15 & -18.1 & 31 & -17.3 \\
\hline NOV 05 & -20.2 & 20 & -17.5 & APR 05 & -17.4 \\
\hline 10 & -20.5 & 25 & -17.6 & 10 & -17.0 \\
\hline 15 & -19.9 & 31 & -17.4 & 15 & -17.6 \\
\hline 20 & -20.0 & AUG 05 & -17.5 & 20 & -17.6 \\
\hline 25 & -20.1 & 10 & -17.0 & 25 & -17.9 \\
\hline 30 & -20.2 & 15 & -17.5 & 30 & -17.9 \\
\hline DEC 05 & -20.1 & 20 & -16.9 & MAY 05 & -18.0 \\
\hline 10 & -20.1 & 25 & -17.3 & 10 & -18.1 \\
\hline 15 & -19.8 & 31 & -17.2 & 15 & -18.3 \\
\hline 20 & -19.7 & SEP 05 & -17.4 & 20 & -18.0 \\
\hline 25 & -19.7 & 10 & -17.2 & 25 & -18.2 \\
\hline 31 & -19.8 & 15 & -17.4 & 31 & -18.4 \\
\hline JAN 05, 1981 & -19.9 & 20 & -17.2 & JUN 05 & -18.4 \\
\hline 10 & -19.5 & 25 & -17.5 & 10 & -18.4 \\
\hline 15 & -19.3 & 30 & -18.1 & 15 & -18.6 \\
\hline 20 & -19.5 & OCT 05 & -18.4 & 20 & -18.1 \\
\hline 25 & -19.4 & 10 & -18.4 & 25 & -18.0 \\
\hline 31 & -19.4 & 15 & -18.5 & 30 & -18.5 \\
\hline FEB 05 & -19.8 & 20 & -18.7 & JUL 05 & -18.9 \\
\hline 10 & -19.7 & 25 & -18.8 & 10 & -18.5 \\
\hline 15 & -19.2 & 31 & -18.6 & 15 & -19.0 \\
\hline 20 & -19.2 & NOV 05 & -18.9 & 20 & -18.6 \\
\hline 25 & -19.2 & 10 & -18.8 & 25 & -18.9 \\
\hline 28 & -19.5 & 15 & -18.9 & 31 & -20.0 \\
\hline MAR 05 & -19.3 & 20 & -18.4 & AUG 05 & -20.0 \\
\hline 10 & -19.4 & 25 & -18.5 & 10 & -19.0 \\
\hline 15 & -19.3 & 30 & -18.3 & 15 & -19.5 \\
\hline 20 & -19.3 & DEC 05 & -18.4 & 20 & -19.4 \\
\hline 25 & -18.8 & 10 & -18.4 & 25 & -19.5 \\
\hline 31 & -18.7 & 15 & -18.5 & 31 & -20.9 \\
\hline APR 05 & -19.0 & 20 & -18.5 & SEP 05 & -21.0 \\
\hline 10 & -19.0 & 25 & -18.5 & 10 & -21.0 \\
\hline 15 & -19.0 & 31 & -19.4 & 15 & -21.4 \\
\hline 20 & -18.8 & JAN 05, 1982 & -17.8 & 20 & -21.5 \\
\hline 25 & -18.7 & 10 & -17.5 & 25 & -21.6 \\
\hline 30 & -18.7 & 15 & -17.4 & 30 & -21.4 \\
\hline MAY 05 & -18.4 & 20 & -17.6 & OCT 05 & -21.5 \\
\hline 10 & -18.8 & 25 & -17.2 & 10 & -21.5 \\
\hline 15 & -18.1 & 31 & -17.2 & 15 & -21.6 \\
\hline 20 & -18.3 & FEB 05 & -17.2 & 20 & -21.8 \\
\hline 25 & -18.5 & 10 & -16.6 & 25 & -21.5 \\
\hline 31 & -18.5 & 15 & -16.5 & 31 & -21.3 \\
\hline JUN 05 & -18.4 & 20 & -17.0 & NOV 05 & -21.2 \\
\hline 10 & -18.5 & 25 & -17.1 & 10 & -21.0 \\
\hline 15 & -18.7 & 28 & -17.3 & 15 & -21.0 \\
\hline 20 & -18.3 & MAR 05 & -17.4 & 20 & -21.0 \\
\hline 25 & -18.2 & 10 & -17.2 & 25 & -20.9 \\
\hline
\end{tabular}


Table 3.-Water levels in selected wells-Continued

\begin{tabular}{|c|c|c|c|c|c|}
\hline Date & Water level & Date & Water level & Date & Water level \\
\hline \multicolumn{2}{|c|}{ (A-12-1)29cab-1-Continued } & \multicolumn{2}{|c|}{ (A-12-1)29cab-1-Continued } & \multicolumn{2}{|c|}{ (A-12-1)29cab-1-Continued } \\
\hline NOV 30,1982 & -20.8 & AUG 15, 1983 & -22.9 & APR 30,1984 & -20.6 \\
\hline DEC 05 & -20.7 & 20 & -22.4 & MAY 05 & -20.6 \\
\hline 10 & -20.8 & 25 & -22.7 & 10 & -20.8 \\
\hline 15 & -20.8 & 31 & -23.0 & 15 & -20.8 \\
\hline 20 & -20.4 & SEP 05 & -23.0 & 20 & -21.0 \\
\hline 25 & -20.4 & 10 & -23.6 & 25 & -21.2 \\
\hline 31 & -20.3 & 15 & -23.6 & 31 & -20.5 \\
\hline JAN 05, 1983 & -20.4 & 20 & -23.3 & JUN 05 & -20.8 \\
\hline 10 & -20.2 & 25 & -23.0 & 10 & -21.1 \\
\hline 15 & -20.2 & 30 & -23.1 & 15 & -22.4 \\
\hline 20 & -20.2 & ОСТ 05 & -23.0 & 20 & -22.0 \\
\hline 25 & -20.1 & 10 & -23.0 & 25 & -22.0 \\
\hline 31 & -20.0 & 15 & -23.1 & 30 & -21.0 \\
\hline FEB 05 & -20.2 & 20 & -23.4 & JUL 05 & -21.8 \\
\hline 10 & -19.9 & 25 & -23.3 & 10 & -22.1 \\
\hline 15 & -20.0 & 31 & -22.7 & 15 & -22.4 \\
\hline 20 & -19.8 & NOV 05 & -22.9 & 20 & -21.8 \\
\hline 25 & -20.0 & 10 & -22.6 & 25 & -22.3 \\
\hline 28 & -19.8 & 15 & -22.7 & 31 & -23.2 \\
\hline MAR 05 & -19.5 & 20 & -22.4 & AUG 05 & -23.4 \\
\hline 10 & -19.6 & 25 & -22.5 & 10 & -23.1 \\
\hline 15 & -19.7 & 30 & -22.3 & 15 & -22.5 \\
\hline 20 & -19.7 & DEC 05 & -22.5 & 20 & -23.2 \\
\hline 25 & -19.6 & 10 & -22.0 & 25 & -22.9 \\
\hline 31 & -19.6 & 15 & -22.0 & 31 & -23.4 \\
\hline APR 05 & -19.7 & 20 & -21.7 & SEP 05 & -23.6 \\
\hline 10 & -19.4 & 25 & -21.6 & 10 & -23.6 \\
\hline 15 & -19.8 & 31 & -21.3 & 15 & -23.5 \\
\hline 20 & -19.5 & JAN 05, 1984 & -21.3 & 20 & -23.5 \\
\hline 25 & -19.4 & 10 & -21.4 & 25 & -23.6 \\
\hline 30 & -19.4 & 15 & -21.2 & 29 & -23.5 \\
\hline MAY 05 & -19.6 & 20 & -21.5 & 30 & -23.5 \\
\hline 10 & -19.6 & 25 & -21.0 & OCT 23 & -23.7 \\
\hline 15 & -19.6 & 31 & -21.0 & NOV 29 & -22.9 \\
\hline 20 & -19.7 & FEB 05 & -21.3 & DEC 28 & -22.2 \\
\hline 25 & -20.1 & 10 & -21.1 & JAN 29, 1985 & -21.4 \\
\hline 31 & -20.1 & 15 & -20.9 & FEB 28 & -20.9 \\
\hline JUN 05 & -20.1 & 20 & -20.9 & MAR 28 & -20.4 \\
\hline 10 & -20.8 & 25 & -20.7 & APR 28 & -20.0 \\
\hline 15 & -20.7 & 29 & -20.8 & JUN 02 & -19.8 \\
\hline 20 & -20.7 & MAR 05 & -21.3 & 26 & -19.3 \\
\hline 25 & -20.7 & 10 & -20.5 & JUL 26 & -19.1 \\
\hline 30 & -20.7 & 15 & -21.0 & AUG 30 & -18.8 \\
\hline JUL 05 & -21.3 & 20 & -21.0 & SEP 19 & -20.1 \\
\hline 10 & -20.8 & 25 & -20.4 & OCT 21 & -20.4 \\
\hline 15 & -21.3 & 31 & -20.1 & NOV 19 & -19.7 \\
\hline 20 & -21.0 & APR 05 & -20.4 & DEC 20 & -19.2 \\
\hline 25 & -21.5 & 10 & -20.2 & JAN 30, 1986 & -18.9 \\
\hline 31 & -21.8 & 15 & -20.5 & FEB 27 & -18.7 \\
\hline AUG 05 & -21.9 & 20 & -20.5 & MAR 28 & -18.8 \\
\hline 10 & -22.0 & 25 & -20.6 & APR 25 & -19.3 \\
\hline
\end{tabular}




\section{Table 3.-Water levels in selected wells-Continued}

\begin{tabular}{|c|c|c|c|c|c|}
\hline Date & Water level & Date & Water level & Date & Water level \\
\hline \multicolumn{2}{|c|}{ (A-12-1)29cab-1-Continued } & \multicolumn{2}{|c|}{ (A-12-1)29cab-1-Continued } & \multicolumn{2}{|c|}{ (A-12-1)34ccc-1-Continued } \\
\hline MAY 30,1986 & -19.3 & MAR 06, 1991 & -12.3 & MAR 13, 1974 & 34.91 \\
\hline JUN 27 & -19.3 & & & SEP 11 & 32.14 \\
\hline JUL 25 & -20.4 & (A-12-1)31dab-2 & & MAR 12,1975 & 33.24 \\
\hline AUG 29 & -18.0 & Altitude $\mathbf{4 , 4 3 0}$ & & OCT 15 & 29.78 \\
\hline SEP 29 & -19.0 & MAR 09, 1977 & -38.4 & MAR 15 & 32.73 \\
\hline JAN 29, 1987 & -17.2 & SEP 29 & -36.2 & SEP 13 & 30.28 \\
\hline FEB 26 & -16.8 & MAR 09, 1978 & -36.0 & MAR 08, 1977 & 33.16 \\
\hline MAR 30 & -16.2 & SEP 20 & -38.1 & OCT 04 & 39.17 \\
\hline APR 29 & -15.3 & MAR 12, 1979 & -37.1 & MAR 08, 1978 & 38.56 \\
\hline MAY 29 & -15.9 & SEP 20 & -34.5 & SEP 18 & 36.71 \\
\hline JUN 29 & -15.0 & MAR 10,1980 & -36.4 & MAR 12, 1979 & 36.15 \\
\hline JUL 31 & -15.3 & SEP 17 & -39.3 & SEP 17 & 36.66 \\
\hline AUG 26 & -15.2 & MAR 02, 1981 & -38.1 & MAR 10,1980 & 36.66 \\
\hline SEP 30 & -15.1 & SEP 17 & -33.6 & SEP 17 & 33.51 \\
\hline Ост 30 & -15.2 & MAR 08, 1982 & -35.6 & MAR 02, 1981 & 34.55 \\
\hline NOV 30 & -14.9 & SEP 08 & -42.8 & SEP 17 & 40.14 \\
\hline DEC 29 & -14.9 & MAR 02, 1983 & -39.3 & MAR 08, 1982 & 36.26 \\
\hline JAN 29, 1988 & -14.3 & AUG 31 & -47.1 & SEP 08 & 31.63 \\
\hline FEB 26 & -14.2 & MAR 02, 1984 & -39.8 & MAR 02, 1983 & 31.35 \\
\hline MAR 31 & -14.1 & SEP 07 & -43.6 & AUG 31 & 27.98 \\
\hline APR 28 & -14.2 & MAR 04, 1985 & -39.8 & MAR 02, 1984 & 29.12 \\
\hline MAY 31 & -13.8 & SEP 19 & -38.2 & SEP 07 & 23.98 \\
\hline JUL 28 & -11.3 & MAR 11,1986 & -37.7 & MAR 04, 1985 & 28.91 \\
\hline AUG 29 & -11.5 & MAR 10,1987 & -37.8 & SEP 19 & 31.08 \\
\hline OCT 05 & -12.4 & MAR 03, 1988 & -37.8 & MAR 10, 1986 & 33.68 \\
\hline 28 & -12.6 & SEP 23 & -33.8 & SEP 29 & 26.93 \\
\hline DEC 29 & -13.4 & MAR 15, 1989 & -32.4 & MAR 05,1987 & 31.48 \\
\hline FEB 22, 1989 & -12.6 & SEP 19 & -35.5 & MAR 08, 1988 & 38.18 \\
\hline MAR 31 & -12.9 & MAR 01, 1990 & -33.2 & SEP 23 & 42.65 \\
\hline APR 28 & -13.0 & APR 27 & -32.6 & MAR 08, 1989 & 40.75 \\
\hline MAY 26 & -13.0 & OCT 29 & -31.0 & SEP 19 & 40.36 \\
\hline JUN 29 & -12.6 & NOV 28 & -28.6 & MAR 01, 1990 & 39.61 \\
\hline JUL 28 & -12.5 & JAN 16, 1991 & -26.0 & JUN 26 & 40.68 \\
\hline AUG 31 & -12.5 & MAR 07 & -26.8 & JUL 26 & 43.19 \\
\hline SEP 28 & -13.7 & & & AUG 28 & 44.75 \\
\hline ОСТ 30 & -13.7 & (A-12-1)34ccc-1 & & SEP 27 & 46.00 \\
\hline NOV 29 & -13.9 & Altitude $\mathbf{4 , 5 3 0}$ & & ОСТ 29 & 44.56 \\
\hline DEC 29 & -13.7 & AUG 05, 1969 & 33.31 & NOV 29 & 43.56 \\
\hline FEB 08, 1990 & -13.4 & SEP 04 & 34.38 & JAN 04, 1991 & 43.55 \\
\hline 28 & -13.0 & OCT 09 & 34.18 & MAR 08 & 44.09 \\
\hline APR 27 & -13.0 & NOV 07 & 31.56 & & \\
\hline MAY 29 & -13.0 & DEC 02 & 31.84 & $(A-13-1) 29 a d c-1$ & \\
\hline JUN 25 & -12.9 & JAN 07, 1970 & 32.94 & Altitude 4,490 & \\
\hline 29 & -12.4 & FEB 09 & 33.68 & AUG 04, 1969 & -3.4 \\
\hline JUL 25 & -12.2 & MAR 04 & 34.46 & SEP 04 & -0.3 \\
\hline AUG 28 & -11.0 & SEP 02 & 34.48 & OCT 08 & -2.6 \\
\hline SEP 27 & -10.8 & MAR 01, 1971 & 34.66 & NOV 06 & -1.7 \\
\hline OCT 29 & -12.7 & MAR 01, 1972 & 32.07 & DEC 03 & .19 \\
\hline NOV 28 & -12.8 & SEP 11 & 28.78 & JAN 06, 1970 & 2.40 \\
\hline DEC 20 & -13.4 & MAR 02, 1973 & 32.74 & FEB 10 & 4.11 \\
\hline JAN 16, 1991 & -12.6 & SEP 13 & 32.63 & MAR 03 & 5.26 \\
\hline
\end{tabular}


Table 3.-Water levels in selected wells-Continued

\begin{tabular}{|c|c|c|c|c|c|}
\hline Date & Water level & Date & Water level & Date & Water level \\
\hline \multicolumn{2}{|c|}{ (A-13-1)29adc-1-Continued } & \multicolumn{2}{|c|}{ (A-14-1)6ccc-1-Continued } & \multicolumn{2}{|c|}{ (A-14-1)11dbb-1-Continued } \\
\hline SEP 02,1970 & 1.08 & JAN 06, 1970 & 4.90 & MAR 04, 1987 & 58.95 \\
\hline MAR 01, 1971 & 4.30 & MAR 03, 1970 & 6.00 & SEP 16 & 63.19 \\
\hline SEP 01 & -4.5 & MAR 12 & 4.20 & MAR 04, 1988 & 70.10 \\
\hline MAR 01, 1972 & 3.84 & SEP 01, 1971 & 4.97 & 07,1989 & 60.34 \\
\hline SEP 12 & -6.8 & MAR 01, 1972 & 1.94 & & \\
\hline MAR 01, 1973 & 4.33 & SEP 12 & 6.09 & $(A-14-1) 14 c c c-1$ & \\
\hline SEP 12 & -2.9 & MAR 01, 1973 & 5.54 & Altitude $\mathbf{4 , 5 3 0}$ & \\
\hline MAR 14, 1974 & 4.99 & SEP 12 & 4.74 & SEP 21,1979 & 72.85 \\
\hline SEP 13 & -4.3 & MAR 13, 1974 & 3.46 & MAR 11, 1980 & 71.59 \\
\hline MAR 10, 1975 & 4.73 & SEP 13 & 5.84 & SEP 18 & 68.06 \\
\hline OCT 14 & -4.3 & MAR 10, 1975 & 7.30 & MAR 04, 1981 & 72.17 \\
\hline MAR 16, 1976 & 4.74 & OCT 14 & 5.31 & MAR 08, 1982 & 70.73 \\
\hline SEP 15 & -3.5 & MAR 16, 1976 & 4.70 & MAR 03, 1983 & 68.52 \\
\hline MAR 09, 1977 & 5.51 & SEP 15 & 5.41 & SEP 02 & 58.87 \\
\hline SEP 29 & 8.29 & MAR 09, 1977 & 5.39 & MAR 05, 1984 & 66.25 \\
\hline MAR 09, 1978 & 10.34 & SEP 28 & 5.76 & SEP 11 & 58.24 \\
\hline SEP 20 & -1.5 & MAR 09, 1978 & 3.24 & MAR 05, 1985 & 67.97 \\
\hline MAR 13, 1979 & 7.20 & SEP 19 & 5.64 & SEP 05 & 69.43 \\
\hline SEP 20 & 4.50 & MAR 13, 1979 & 4.76 & MAR 10, 1986 & 64.87 \\
\hline MAR 11, 1980 & 8.15 & SEP 20 & 5.73 & SEP 23 & 64.22 \\
\hline SEP 18 & -2.1 & MAR 11,1980 & 1.99 & MAR 04, 1987 & 67.93 \\
\hline MAR 04, 1981 & 7.29 & SEP 18 & 4.35 & MAR 04, 1988 & 72.13 \\
\hline SEP 22 & 5.14 & MAR 04, 1981 & 8.90 & SEP 23 & 81.64 \\
\hline MAR 08, 1982 & 8.95 & SEP 22 & 4.78 & MAR 07, 1989 & 74.30 \\
\hline SEP 09 & -2.5 & MAR 08, 1982 & 6.13 & SEP 18 & 72.84 \\
\hline MAR 03, 1983 & 4.80 & SEP 09 & 4.96 & MAR 01, 1990 & 73.12 \\
\hline SEP 02 & -4.0 & SEP 02, 1983 & 3.90 & APR 26 & 68.97 \\
\hline SEP 11,1984 & -7.0 & SEP 11,1984 & 4.48 & MAY 29 & 67.84 \\
\hline SEP 05, 1985 & -2.5 & SEP 05, 1985 & 3.59 & JUL 25 & 71.61 \\
\hline SEP 29, 1986 & -5.4 & SEP 23, 1986 & 5.85 & SEP 26 & 75.47 \\
\hline SEP 15,1987 & 4.57 & & & NOV 01 & 75.45 \\
\hline SEP 26, 1988 & 9.94 & $(A-14-1) 11 d b b-1$ & & 27 & 74.91 \\
\hline SEP 18,1989 & 5.73 & Altitude 4,530 & & JAN 05, 1991 & 74.98 \\
\hline APR 26, 1990 & 9.85 & MAR 20, 1978 & 63.73 & MAR 08 & 73.48 \\
\hline MAY 30 & 9.00 & SEP 19 & 60.35 & & \\
\hline JUN 26 & 7.93 & MAR 13, 1979 & 63.63 & (A-14-1)22bad-1 & \\
\hline JUL 25 & 12.70 & SEP 20 & 63.65 & Altitude 4,466 & \\
\hline AUG 28 & 15.89 & MAR 11,1980 & 63.20 & AUG 04, 1969 & -15.5 \\
\hline SEP 27 & 14.30 & SEP 18 & 59.08 & SEP 04 & -10.9 \\
\hline OCT 29 & 12.03 & MAR 04, 1981 & 62.67 & ОСТ 08 & -7.4 \\
\hline NOV 27 & 11.67 & SEP 22 & 61.88 & NOV 06 & -5.1 \\
\hline JAN 03, 1991 & 12.34 & MAR 08, 1982 & 61.90 & DEC 03 & -3.9 \\
\hline \multirow[t]{2}{*}{ MAR 08} & 12.53 & SEP 09 & 56.70 & JAN 06, 1970 & -2.8 \\
\hline & & MAR 03, 1983 & 59.18 & FEB 10 & -2.6 \\
\hline$(A-14-1) 6 c c c-1$ & & SEP 02 & 49.90 & MAR 03 & -2.5 \\
\hline Altitude 4,505 & & MAR 05, 1984 & 56.76 & SEP 02 & -10.3 \\
\hline AUG 04, 1969 & 4.77 & SEP 11 & 56.18 & MAR 01, 1971 & -1.0 \\
\hline SEP 04, 1969 & 4.80 & MAR 05, 1985 & 57.70 & SEP 01 & -16.3 \\
\hline ОСТ 08 & 5.00 & SEP 05 & 61.27 & MAR 01, 1972 & -7.3 \\
\hline NOV 06 & 5.62 & MAR 10, 1986 & 57.24 & SEP 12 & -12.0 \\
\hline DEC 03 & 4.85 & SEP 23 & 54.80 & MAR 01, 1973 & -6.6 \\
\hline
\end{tabular}


Table 3.-Water levels in selected wells-Continued

\begin{tabular}{|c|c|c|c|c|c|}
\hline Date & Water level & Date & Water level & Date & Water level \\
\hline \multicolumn{2}{|c|}{ (A-14-1)22bad-1-Continued } & \multicolumn{2}{|c|}{ (A-14-1)34adb-1-Continued } & \multicolumn{2}{|c|}{ (A-14-1)34adb-1-Continued } \\
\hline SEP 12,1973 & -9.8 & JAN 06, 1970 & 15.90 & JAN 03, 1991 & 20.40 \\
\hline MAR 13, 1974 & -7.4 & FEB 10 & 13.99 & MAR 08 & 20.20 \\
\hline SEP 13 & -10.4 & MAR 03 & 12.75 & & \\
\hline MAR 10, 1975 & -3.6 & SEP 02 & 11.56 & (B-10-1)11dad-1 & \\
\hline OCT 14 & -9.3 & MAR 01, 1971 & 10.99 & Altitude 4,680 & \\
\hline SEP 15,1976 & -9.8 & SEP 01 & 6.27 & APR 25, 1990 & 15.89 \\
\hline MAR 09, 1977 & -3.5 & MAR 01, 1972 & 9.14 & MAY 29 & 16.30 \\
\hline SEP 28 & -2.7 & SEP 12 & 10.29 & JUN 26 & 16.92 \\
\hline MAR 09, 1978 & -2.5 & MAR 01, 1973 & 17.43 & AUG 27 & 17.48 \\
\hline SEP 19 & -4.6 & SEP 12 & 9.43 & SEP 25 & 18.16 \\
\hline MAR 13, 1979 & -1.0 & $\operatorname{MAR} 13,1974$ & 8.98 & OCT 29 & 19.22 \\
\hline SEP 20 & -1.0 & SEP 13 & 8.97 & NOV 26 & 19.83 \\
\hline MAR 11, 1980 & -2.2 & MAR 10, 1975 & 12.52 & JAN 03, 1991 & 20.56 \\
\hline SEP 18 & -4.3 & OCT 14 & 11.45 & MAR 06 & 20.75 \\
\hline MAR 17,1981 & -2.0 & MAR 16, 1976 & 11.45 & & \\
\hline SEP 22 & -4.0 & SEP 15 & 17.18 & $(B-11-1) 9 c d b-1$ & \\
\hline MAR 08, 1982 & -4.3 & MAR 09, 1977 & 29.80 & Altitude 4,420 & \\
\hline SEP 09 & -4.5 & SEP 28 & 23.43 & AUG 05, 1969 & -5.4 \\
\hline MAR 03, 1983 & -3.8 & MAR 09, 1978 & 14.91 & SEP 04 & -5.1 \\
\hline SEP 02 & -8.0 & SEP 19 & 15.23 & OCT 09 & -5.2 \\
\hline MAR 05, 1984 & -6.1 & MAR 13, 1979 & 26.70 & NOV 07 & -5.2 \\
\hline SEP 11 & -7.6 & SEP 20 & 17.42 & DEC 02 & -5.0 \\
\hline MAR 05, 1985 & -4.4 & MAR 11, 1980 & 14.66 & JAN 06, 1970 & -4.6 \\
\hline SEP 05 & -3.5 & SEP 18 & 12.95 & FEB 09 & -6.1 \\
\hline $\operatorname{MAR} 10,1986$ & -5.9 & MAR 04, 1981 & 23.22 & MAR 04 & -5.6 \\
\hline SEP 29 & -6.0 & SEP 22 & 13.63 & MAR 12, 1971 & -6.3 \\
\hline MAR 04, 1987 & -4.5 & MAR 08, 1982 & 14.00 & SEP 01 & -7.2 \\
\hline SEP 15 & -0.4 & SEP 09 & 8.60 & MAR 01, 1972 & -6.8 \\
\hline MAR 04, 1988 & -2.1 & MAR 03, 1983 & 11.40 & SEP 12 & -6.4 \\
\hline SEP 23 & 1.80 & SEP 02 & 8.97 & MAR 02, 1973 & -6.6 \\
\hline MAR 07, 1989 & .23 & MAR 02, 1984 & 12.32 & SEP 13 & -5.9 \\
\hline SEP 18 & -1.1 & SEP 11 & 10.34 & MAR 13, 1974 & -5.9 \\
\hline MAR 01, 1990 & -1.0 & MAR 05, 1985 & 12.86 & SEP 11 & -6.6 \\
\hline APR 26 & -3.2 & SEP 05 & 13.45 & MAR 10, 1975 & -6.8 \\
\hline MAY 30 & -4.1 & MAR 10, 1986 & 7.32 & OCT 15 & -6.8 \\
\hline JUN 28 & -4.8 & SEP 23 & 11.78 & MAR 15, 1976 & -6.8 \\
\hline JUL 24 & -1.9 & MAR 04, 1987 & 15.94 & SEP 13 & -6.3 \\
\hline AUG 28 & 1.26 & SEP 15 & 17.74 & MAR 08, 1977 & -7.7 \\
\hline SEP 26 & 1.28 & MAR 04, 1988 & 19.39 & SEP 28 & -7.7 \\
\hline NOV 01 & 1.24 & SEP 23 & 38.08 & MAR 08, 1978 & -7.1 \\
\hline 29 & .79 & MAR 07, 1989 & 18.95 & SEP 19 & -6.4 \\
\hline JAN 04, 1991 & .88 & SEP 18 & 15.55 & MAR 12, 1979 & -7.4 \\
\hline MAR 08 & -0.7 & MAR 01, 1990 & 18.33 & SEP 18 & -7.3 \\
\hline & & APR 26 & 15.03 & MAR 10, 1980 & -6.8 \\
\hline$(A-14-1) 34 a d b-1$ & & MAY 29 & 12.25 & SEP 17 & -6.6 \\
\hline Altitude 4,540 & & JUN 26 & 9.74 & MAR 02, 1981 & -6.8 \\
\hline AUG 04, 1969 & 8.66 & JUL 25 & 11.01 & SEP 17 & -6.5 \\
\hline SEP 04 & 8.39 & AUG 27 & 21.50 & MAR 08, 1982 & -6.5 \\
\hline OCT 08 & 10.95 & SEP 26 & 19.02 & SEP 08 & -7.5 \\
\hline NOV 06 & 13.08 & NOV 01 & 18.98 & MAR 02, 1983 & -6.9 \\
\hline DEC 03 & 14.66 & 27 & 19.19 & AUG 31 & -6.8 \\
\hline
\end{tabular}


Table 3.-Water levels in selected wells-Continued

\begin{tabular}{|c|c|c|c|c|c|}
\hline Date & Water level & Date & Water level & Date & Water level \\
\hline \multicolumn{2}{|c|}{ (B-11-1)9cdb-1-Continued } & \multicolumn{2}{|c|}{ (B-11-1)14aaa-3-Continued } & \multicolumn{2}{|c|}{ (B-11-1)35cca-1-Continued } \\
\hline MAR 02, 1984 & -7.4 & SEP 17,1979 & -38.2 & SEP 19,1989 & -5.5 \\
\hline SEP 07 & -7.2 & MAR 10, 1980 & -34.5 & MAR 01, 1990 & -5.6 \\
\hline MAR 04, 1985 & -7.3 & SEP 17 & -35.8 & APR 25 & -5.1 \\
\hline SEP 05 & -6.9 & MAR 02, 1981 & -33.6 & MAY 29 & -5.6 \\
\hline MAR 10, 1986 & -7.7 & SEP 17 & -28.9 & JUN 28 & -4.0 \\
\hline SEP 23 & -6.3 & MAR 08, 1982 & -25.4 & JUL 24 & -4.3 \\
\hline MAR 05, 1987 & -7.1 & & & AUG 27 & -3.3 \\
\hline SEP 15 & -6.5 & (B-11-1)35caa-2 & & SEP 25 & -3.9 \\
\hline MAR 03, 1988 & -7.3 & Altltude $\mathbf{4 , 4 7 0}$ & & OCT 29 & -3.4 \\
\hline SEP 23 & -6.7 & AUG 05, 1969 & -16.0 & NOV 28 & -4.1 \\
\hline MAR 06, 1989 & -6.5 & SEP 04 & -15.8 & JAN 17, 1991 & -4.3 \\
\hline SEP 19 & -6.6 & ОСТ 09 & -16.0 & MAR 06 & -3.6 \\
\hline MAR 01, 1990 & -6.1 & NOV 07 & -16.6 & & \\
\hline APR 25 & -5.8 & DEC 02 & -16.3 & $(B-12-1) 8 c d b-2$ & \\
\hline MAY 29 & -6.3 & JAN 07, 1970 & -13.8 & Altitude $\mathbf{4 , 4 3 0}$ & \\
\hline JUN 28 & -6.3 & FEB 09 & -14.8 & AUG 05, 1969 & -10.9 \\
\hline JUL 24 & -6.3 & MAR 04 & -15.0 & SEP 04 & -10.4 \\
\hline AUG 27 & -6.1 & SEP 03 & -14.8 & ОСТ 08 & -10.6 \\
\hline SEP 25 & -6.4 & MAR 01, 1971 & -15.6 & NOV 06 & -10.2 \\
\hline ОСТ 29 & -6.6 & SEP 01 & -17.7 & DEC 03 & -9.6 \\
\hline NOV 28 & -6.4 & MAR 01, 1972 & -16.8 & JAN 06, 1970 & -9.4 \\
\hline JAN 17, 1991 & -6.8 & SEP 12 & -17.9 & FEB 10 & -9.4 \\
\hline \multirow[t]{2}{*}{ MAR 06} & -5.5 & MAR 02, 1973 & -16.5 & MAR 03 & -10.6 \\
\hline & & SEP 13 & -15.9 & SEP 03 & -9.1 \\
\hline (B-11-1)14aaa-3 & & MAR 13, 1974 & -14.0 & MAR 01, 1971 & -7.2 \\
\hline Altitude $\mathbf{4 , 4 3 0}$ & & SEP 11 & -16.9 & SEP 01 & -11.1 \\
\hline AUG 04, 1969 & -37.5 & MAR 12,1975 & -16.4 & MAR 01, 1972 & -11.3 \\
\hline SEP 04 & -37.5 & OCT 15 & -17.5 & SEP 12 & -10.7 \\
\hline ОСТ 09 & -40.5 & MAR 15, 1976 & -16.4 & MAR 01, 1973 & -7.9 \\
\hline NOV 07 & -43.2 & MAR 08, 1977 & -12.9 & SEP 12 & -11.3 \\
\hline DEC 02 & -41.1 & SEP 28 & -8.7 & MAR 13,1974 & -10.3 \\
\hline JAN 07, 1970 & -41.0 & MAR 08, 1978 & -12.7 & SEP 11 & -10.6 \\
\hline FEB 09 & -40.7 & SEP 19 & -13.0 & MAR 10, 1975 & -10.1 \\
\hline MAR 04 & -39.8 & MAR 10, 1980 & 0. & OCT 14 & -9.7 \\
\hline SEP 03 & -41.3 & & & MAR 15, 1976 & -9.2 \\
\hline MAR 01, 1971 & -39.0 & (B-11-1)35cca-1 & & SEP 14 & -10.2 \\
\hline SEP 01 & -44.3 & Altitude $\mathbf{4 , 4 7 5}$ & & MAR 08, 1977 & -10.0 \\
\hline MAR 01, 1972 & -41.8 & FEB 23,1983 & -12.3 & SEP 28 & -8.9 \\
\hline SEP 21 & -41.2 & MAR 02 & -12.3 & MAR 09, 1978 & -8.5 \\
\hline MAR 02, 1973 & -40.9 & AUG 31 & -14.3 & SEP 19 & -8.6 \\
\hline SEP 13 & -41.4 & MAR 02, 1984 & -12.9 & MAR 12, 1979 & -9.0 \\
\hline MAR 13, 1974 & -40.1 & SEP 07 & -12.7 & SEP 17 & -9.5 \\
\hline SEP 11 & -42.2 & MAR 04, 1985 & -13.4 & MAR 10, 1980 & -8.6 \\
\hline MAR 12,1975 & -40.8 & SEP 05 & -10.0 & SEP 17 & -9.4 \\
\hline OCT 15 & -39.9 & MAR 10, 1986 & -9.5 & MAR 02, 1981 & -9.3 \\
\hline MAR 19, 1976 & -40.6 & SEP 23 & -9.8 & SEP 17 & -8.0 \\
\hline MAR 08, 1977 & -39.8 & MAR 05, 1987 & -9.7 & MAR 08, 1982 & -8.0 \\
\hline SEP 28 & -39.1 & SEP 15 & -7.2 & SEP 08 & -10.6 \\
\hline MAR 08, 1978 & -39.4 & MAR 03, 1988 & -6.8 & MAR 02, 1983 & -10.3 \\
\hline SEP 19 & -39.2 & SEP 23 & -4.5 & AUG 31 & -15.8 \\
\hline MAR 20, 1979 & -39.2 & MAR 06, 1989 & -4.9 & MAR 02, 1984 & -14.2 \\
\hline
\end{tabular}




\section{Table 3.-Water levels in selected wells-Continued}

\begin{tabular}{|c|c|c|c|c|c|}
\hline Date & Water level & Date & Water level & Date & Water level \\
\hline \multicolumn{2}{|c|}{ (B-12-1)8cdb-2-Continued } & \multicolumn{2}{|c|}{ (B-12-1)26cca-1-Continued } & \multicolumn{2}{|c|}{ (B-13-1)10bba-1-Continued } \\
\hline SEP 07, 1984 & -15.2 & SEP 08, 1982 & -12.5 & MAR 06, 1989 & 4.55 \\
\hline MAR 04, 1985 & -13.2 & MAR 02, 1983 & -11.9 & SEP 18 & 4.64 \\
\hline SEP 05 & -12.9 & AUG 31 & -12.7 & MAR 01, 1990 & 4.82 \\
\hline MAR 10, 1986 & -12.4 & MAR 02, 1984 & -12.9 & APR 25 & 4.88 \\
\hline SEP 23 & -12.3 & SEP 07 & -12.6 & MAY 29 & 4.36 \\
\hline MAR 04, 1987 & -11.5 & MAR 04, 1985 & -12.8 & JUN 28 & 4.47 \\
\hline SEP 15 & -11.6 & SEP 19 & -12.2 & JUL 24 & 4.49 \\
\hline MAR 04, 1988 & -9.6 & MAR 10, 1986 & -13.9 & AUG 27 & 4.60 \\
\hline SEP 23 & -8.6 & SEP 29 & -13.2 & SEP 26 & 4.89 \\
\hline MAR 06, 1989 & -8.1 & MAR 05, 1987 & -13.2 & OCT 30 & 4.80 \\
\hline SEP 29 & -8.4 & SEP 15 & -12.4 & NOV 26 & 5.13 \\
\hline MAR 01, 1990 & -7.4 & MAR 03, 1988 & -12.1 & JAN 03, 1991 & 5.17 \\
\hline APR 25 & -7.0 & SEP 23 & -11.2 & MAR 07 & 4.87 \\
\hline MAY 29 & -7.7 & MAR 15, 1989 & -11.4 & & \\
\hline JUN 28 & -7.6 & SEP 18 & -11.7 & (B-13-1)28abb-1 & \\
\hline JUL 24 & -7.5 & MAR 01, 1990 & -12.0 & Altitude $\mathbf{4 , 4 6 0}$ & \\
\hline AUG 27 & -7.2 & APR 25 & -11.9 & MAY 29, 1990 & 9.67 \\
\hline SEP 25 & -7.2 & MAY 29 & -12.2 & JUN 27 & 9.87 \\
\hline NOV 30 & -6.0 & JUN 28 & -12.6 & JUL 24 & 10.01 \\
\hline JAN 16, 1991 & -5.8 & JUL 24 & -12.4 & AUG 27 & 9.98 \\
\hline \multirow[t]{2}{*}{ MAR 09} & -5.5 & AUG 27 & -11.8 & SEP 26 & 10.68 \\
\hline & & SEP 25 & -12.2 & ОСТ 29 & 11.45 \\
\hline (B-12-1)15adc-1 & & OCT 29 & -12.0 & NOV 26 & 11.65 \\
\hline Altitude 4,415 & & NOV 28 & -11.8 & JAN 03, 1991 & 11.96 \\
\hline APR 26, 1990 & -11.5 & JAN 16, 1991 & -11.6 & MAR 07 & 11.68 \\
\hline MAY 30 & -10.8 & MAR 07 & -12.0 & & \\
\hline JUN 28 & -10.9 & & & (B-13-1)30acc-1 & \\
\hline JUL 24 & -11.4 & (B-13-1)10bba-1 & & Altitude $\mathbf{4 , 4 1 0}$ & \\
\hline AUG 27 & -11.4 & Altitude 4,485 & & AUG 05, 1969 & -22.3 \\
\hline SEP 25 & -11.3 & APR 11,1978 & 4.80 & SEP 04 & -21.5 \\
\hline ОСТ 29 & -10.9 & SEP 19 & 5.53 & ОСТ 08 & -22.5 \\
\hline NOV 29 & -11.0 & MAR 13, 1979 & 5.03 & NOV 06 & -22.1 \\
\hline JAN 17, 1991 & -11.2 & SEP 20 & 4.65 & DEC 03 & -23.5 \\
\hline \multirow[t]{2}{*}{ MAR 07} & -10.8 & MAR 11,1980 & 4.82 & JAN 06, 1970 & -22.2 \\
\hline & & SEP 18 & 4.65 & FEB 10 & -23.1 \\
\hline (B-12-1)15adc-2 & & MAR 04, 1981 & 4.79 & MAR 03 & -23.7 \\
\hline Altitude 4,415 & & SEP 22 & 5.77 & SEP 02 & -19.9 \\
\hline ОСТ 29,1990 & -7.1 & MAR 08, 1982 & 4.43 & MAR 01, 1971 & -21.6 \\
\hline NOV 29 & -6.9 & SEP 09 & 3.93 & SEP 01 & -22.9 \\
\hline JAN 17, 1991 & -7.4 & MAR 03, 1983 & 3.86 & MAR 01, 1972 & -23.9 \\
\hline \multirow{2}{*}{ MAR 07} & -7.3 & SEP 02 & 4.66 & SEP 12 & -21.8 \\
\hline & & MAR 05, 1984 & 3.82 & MAR 01, 1973 & -21.8 \\
\hline (B-12-1)26cca-1 & & SEP 11 & 4.30 & SEP 12 & -22.3 \\
\hline Altitude 4,420 & & MAR 05, 1985 & 4.02 & MAR 13, 1974 & -19.9 \\
\hline MAR 20, 1979 & -9.6 & SEP 05 & 4.55 & SEP 11 & -20.2 \\
\hline SEP 20 & -7.3 & MAR 10, 1986 & 4.00 & MAR 10, 1975 & -20.1 \\
\hline MAR 10, 1980 & -12.5 & SEP 23 & 4.16 & OCT 14 & -21.7 \\
\hline SEP 17 & -5.8 & MAR 04, 1987 & 4.63 & MAR 15, 1976 & -20.2 \\
\hline MAR 20, 1981 & -12.9 & SEP 15 & 4.96 & SEP 14 & -22.1 \\
\hline SEP 17 & -10.7 & MAR 04, 1988 & 4.75 & MAR 08, 1977 & -18.8 \\
\hline MAR 08, 1982 & -11.1 & SEP 23 & 4.75 & SEP 28 & -19.7 \\
\hline
\end{tabular}


Table 3.-Water levels in selected wells-Continued

\begin{tabular}{|c|c|c|c|c|c|}
\hline Date & Water level & Date & Water level & Date & Water level \\
\hline \multicolumn{2}{|c|}{ (B-13-1)30acc-1-Continued } & \multicolumn{2}{|c|}{ (B-14-1)31aaa-1-Continued } & \multicolumn{2}{|c|}{ (B-14-2)27ddd-1-Continued } \\
\hline MAR 09, 1978 & -20.5 & MAR 05, 1985 & 9.93 & SEP 23,1988 & .38 \\
\hline SEP 19 & -20.6 & SEP 05 & 10.22 & MAR 22, 1989 & 9.29 \\
\hline MAR 12, 1979 & -19.8 & MAR 10, 1986 & 5.82 & SEP 18 & 11.62 \\
\hline SEP 19 & -20.7 & SEP 23 & 11.39 & MAR 01, 1990 & 15.01 \\
\hline MAR 10, 1980 & -19.5 & MAR 04, 1987 & 11.35 & APR 25 & 14.86 \\
\hline SEP 17 & -21.4 & & & MAY 29 & 15.00 \\
\hline MAR 02, 1981 & -20.2 & (B-14-2)27ddd-1 & & JUN 27 & 13.70 \\
\hline SEP 17 & -19.0 & Altitude 4,890 & & JUL 24 & 15.24 \\
\hline MAR 08, 1982 & -19.4 & AUG 04, 1969 & 8.69 & AUG 27 & 16.10 \\
\hline SEP 08 & -24.1 & SEP 04 & 9.97 & SEP 26 & 16.70 \\
\hline MAR 02, 1983 & -22.6 & OCT 08 & 13.75 & ОСТ 30 & 16.75 \\
\hline AUG 31 & -27.7 & NOV 06 & 15.30 & NOV 26 & 18.07 \\
\hline MAR 02, 1984 & -23.2 & DEC 03 & 15.34 & JAN 04, 1991 & 18.52 \\
\hline SEP 07 & -23.6 & JAN 06, 1970 & 13.06 & MAR 07 & 15.95 \\
\hline MAR 04, 1985 & -22.2 & FEB 10 & 9.95 & & \\
\hline SEP 05 & -22.6 & MAR 03 & 9.93 & $(B-15-1) 34 c c c-1$ & \\
\hline MAR 10, 1986 & -23.5 & MAR 12, 1971 & 11.12 & Altitude 4,510 & \\
\hline SEP 23 & -19.0 & SEP 01 & 10.01 & AUG 04, 1969 & 5.87 \\
\hline MAR 04, 1987 & -22.3 & MAR 01, 1972 & 11.08 & SEP 04 & 6.04 \\
\hline SEP 15 & -21.5 & SEP 12 & 11.39 & ОСТ 08 & 7.42 \\
\hline MAR 09, 1988 & -21.2 & MAR 01, 1973 & 15.94 & NOV 06 & 8.08 \\
\hline SEP 23 & -19.8 & SEP 12 & 10.15 & DEC 03 & 8.34 \\
\hline MAR 15, 1989 & -23.6 & MAR 13,1974 & 11.30 & JAN 06, 1970 & 9.10 \\
\hline SEP 19 & -20.4 & SEP 13 & 10.86 & FEB 10 & 9.32 \\
\hline MAR 01, 1990 & -19.8 & MAR 10, 1975 & 11.69 & MAR 03 & 9.46 \\
\hline APR 25 & -19.4 & OCT 14 & 11.82 & SEP 02 & 6.09 \\
\hline MAY 29 & -19.0 & MAR 16, 1976 & 11.92 & MAR 01, 1971 & 8.45 \\
\hline JUN 28 & -18.7 & SEP 15 & 11.97 & SEP 01 & 7.19 \\
\hline JUL 24 & -18.7 & MAR 09, 1977 & 16.75 & MAR 01, 1972 & 9.15 \\
\hline AUG 27 & -19.2 & SEP 28 & 16.90 & SEP 12 & 6.80 \\
\hline SEP 25 & -19.2 & MAR 09, 1978 & 11.10 & MAR 01, 1973 & 9.34 \\
\hline OCT 29 & -18.7 & SEP 19 & 13.27 & SEP 12 & 6.67 \\
\hline NOV 29 & -17.5 & MAR 13,1979 & 12.13 & MAR 13,1974 & 8.01 \\
\hline JAN 16, 1991 & -17.2 & SEP 20 & 10.12 & SEP 13 & 6.70 \\
\hline \multirow[t]{2}{*}{ MAR 07} & -17.0 & MAR 11,1980 & 7.97 & MAR 10, 1975 & 8.75 \\
\hline & & SEP 18 & 10.64 & OCT 14 & 7.98 \\
\hline (B-14-1)31aaa-1 & & MAR 04, 1981 & 15.54 & MAR 16, 1976 & 9.25 \\
\hline Altitude 4,790 & & SEP 22 & 13.91 & SEP 15 & 7.06 \\
\hline MAR 20, 1978 & 11.59 & MAR 08, 1982 & 10.77 & MAR 09, 1977 & 9.82 \\
\hline SEP 19 & 11.02 & SEP 09 & 8.88 & SEP 28 & 7.98 \\
\hline MAR 13, 1979 & 12.28 & MAR 03, 1983 & 11.00 & MAR 08, 1978 & 9.11 \\
\hline SEP 20 & 11.09 & SEP 02 & 7.83 & SEP 19 & 6.80 \\
\hline MAR 11,1980 & 10.95 & MAR 05, 1984 & 12.22 & MAR 13,1979 & 7.88 \\
\hline SEP 18 & 9.73 & SEP 11 & 7.82 & SEP 20 & 6.05 \\
\hline MAR 04, 1981 & 11.18 & MAR 05, 1985 & 10.03 & MAR 11,1980 & 7.45 \\
\hline SEP 22 & 13.65 & SEP 05 & 8.52 & SEP 18 & 7.64 \\
\hline MAR 08, 1982 & 11.95 & MAR 10, 1986 & 7.14 & MAR 04, 1981 & 9.68 \\
\hline SEP 09 & 11.48 & SEP 23 & 10.80 & SEP 22 & 7.80 \\
\hline MAR 03, 1983 & 7.34 & MAR 04, 1987 & 12.57 & MAR 08, 1982 & $7.7 \overline{6}$ \\
\hline SEP 02 & 9.88 & SEP 15 & 12.37 & SEP 09 & 7.54 \\
\hline SEP 11,1984 & 9.49 & MAR 04, 1988 & 14.47 & MAR 03, 1983 & 8.74 \\
\hline
\end{tabular}


Table 3.-Water levels in selected wells-Continued

\begin{tabular}{|c|c|}
\hline Date & Water level \\
\hline \multicolumn{2}{|c|}{ (B-14-2)27ddd-1-Continued } \\
\hline SEP 02, 1983 & 7.72 \\
\hline MAR 02, 1984 & 8.98 \\
\hline SEP 11 & 7.72 \\
\hline MAR 05, 1985 & 8.26 \\
\hline SEP 05 & 6.97 \\
\hline MAR 10, 1986 & 7.54 \\
\hline SEP 23 & 7.11 \\
\hline MAR 04, 1987 & 9.91 \\
\hline SEP 17 & 8.45 \\
\hline MAR 04, 1988 & 9.80 \\
\hline SEP 23 & 11.02 \\
\hline MAR 07, 1989 & 9.49 \\
\hline SEP 18 & 8.15 \\
\hline MAY 29, 1990 & 8.60 \\
\hline JUL 11 & 8.12 \\
\hline 24 & 7.95 \\
\hline AUG 27 & 8.08 \\
\hline SEP 26 & 8.73 \\
\hline OCT 30 & 9.40 \\
\hline NOV 26 & 9.65 \\
\hline JAN 03, 1991 & 9.68 \\
\hline MAR 07 & 9.74 \\
\hline \multicolumn{2}{|c|}{ (B-15-1)34ccc-2 } \\
\hline \multicolumn{2}{|l|}{ Altitude 4,515} \\
\hline MAR 11, 1980 & 8.97 \\
\hline SEP 18 & 9.08 \\
\hline MAR 04, 1981 & 11.01 \\
\hline SEP 22 & 9.22 \\
\hline MAR 08, 1982 & 9.88 \\
\hline SEP 09 & 8.95 \\
\hline MAR 03, 1983 & 10.28 \\
\hline SEP 02 & 9.08 \\
\hline SEP 11, 1984 & 9.15 \\
\hline SEP 05, 1985 & 8.33 \\
\hline SEP 29, 1986 & 8.75 \\
\hline SEP 17,1987 & 9.75 \\
\hline SEP 23, 1988 & 7.34 \\
\hline SEP 18, 1989 & 9.28 \\
\hline MAR 01, 1990 & 9.82 \\
\hline JUN 27 & 9.69 \\
\hline JUL 11 & 9.48 \\
\hline 24 & 9.34 \\
\hline AUG 27 & 9.29 \\
\hline SEP 26 & 9.90 \\
\hline OCT 29 & 10.69 \\
\hline NOV 26 & 10.87 \\
\hline JAN 03, 1991 & 10.98 \\
\hline MAR 07 & 11.05 \\
\hline
\end{tabular}

\begin{tabular}{ll}
\hline Date & Water level \\
\hline 13S 38E 03DDB1 & \\
Altitude 4,850 & \\
MAR 08, 1977 & 24.26 \\
SEP 30 & 26.72 \\
MAR 21, 1978 & 24.76 \\
APR 04,1990 & 22.77 \\
MAY 30 & 23.88 \\
JUN 26 & 25.22 \\
JUL 25 & 25.49 \\
AUG 27 & 25.77 \\
SEP 26 & 25.41 \\
OCT 30 & 24.28 \\
NOV 26 & 23.91 \\
JAN 04, 1991 & 23.96 \\
MAR 07 & 23.45 \\
\end{tabular}

$\begin{array}{ll}\text { 13S 40E 30ACB1 } \\ \text { Altitude 5,050 } \\ \text { SEP 08, 1969 } \\ \text { OCT 10 } \\ \text { NOV 13 } & 36.55 \\ \text { DEC 04 } & 27.02 \\ \text { JAN 08, 1970 } & 24.62 \\ \text { FEB 11 } & 23.93 \\ \text { MAR 09 } & 22.95 \\ \text { MAR 08, 1977 } & 20.19 \\ \text { SEP 29 } & 20.66 \\ \text { MAR 21, 1978 } & 19.85 \\ \text { APR 26, 1990 } & 35.39 \\ \text { SEP 26 } & 17.85 \\ \text { NOV 01 } & 30.25 \\ 27 & 51.35 \\ \text { JAN 04, 1991 } & 44.92 \\ \text { MAR 07 } & 42.24 \\ & 39.86 \\ & 34.56\end{array}$

14S 38E 04BBC1

Altitude 4,800

APR 11, $1990 \quad 20.11$

$26 \quad 19.25$

MAY 30

JUN 26

JUL 25

AUG 27

SEP 26

OCT 30

NOV 27

JAN 04, 1991

22.89

MAR 07

22.49

12.28

\begin{tabular}{ll}
\hline Date & Water level \\
\hline 14S 38E 15CDC1 &
\end{tabular}

Altitude 4,800

AUG 11, $1969 \quad 27.89$

SEP $08 \quad 32.62$

$23 \quad 31.84$

OCT $10 \quad 28.70$

NOV $13 \quad 24.06$

DEC $04 \quad 22.68$

JAN 08, $1970 \quad 23.83$

FEB $11 \quad 20.90$

MAR $09 \quad 20.60$

$\begin{array}{ll}18 & 20.67\end{array}$

SEP 24

MAR 23, $1971 \quad 21.37$

SEP $15 \quad 23.68$

MAR 08, $1972 \quad 17.98$

SEP $27 \quad 29.09$

MAR 28, $1973 \quad 20.07$

SEP $26 \quad 30.54$

MAR 20, $1974 \quad 22.15$

SEP $23 \quad 40.20$

MAR 19, $1975 \quad 23.84$

SEP $19 \quad 37.15$

MAR 10, $1976 \quad 21.78$

SEP $09 \quad 31.84$

MAR 08, $1977 \quad 22.06$

JUL $06 \quad 46.02$

AUG $12 \quad 52.58$

$17 \quad 46.30$

SEP $20 \quad 43.02$

$29 \quad 41.53$

NOV $09 \quad 36.41$

DEC 21

FEB 09, $1978 \quad$. $\quad 31.32$

MAR $21 \quad 29.95$

APR $26 \quad 25.20$

MAY $31 \quad 22.60$

JUL 12

AUG $23 \quad 38.43$

SEP $26 \quad 33.81$

NOV $08 \quad 28.85$

DEC $20 \quad 26.26$

JAN 24, $1979 \quad 24.92$

MAR $07 \quad 23.83$

APR 20

MAY 23

JUL $25 \quad 33.76$

SEP $12 \quad 35.05$

OCT $24 \quad 31.39$

DEC 12

FEB 07, $1980 \quad 25.98$

MAR $26 \quad 24.83$ 
Table 3.-Water levels in selected wells-Continued

\begin{tabular}{|c|c|c|c|c|c|}
\hline Date & Water level & Date & Water level & Date & Water level \\
\hline \multicolumn{2}{|c|}{ 14S 38E 15CDC1-Continued } & \multicolumn{2}{|c|}{ 14S 38E 15CDC1-Continued } & \multicolumn{2}{|c|}{ 14S 39E 29DAD1-Continued } \\
\hline MAY 14,1980 & 21.31 & MAR 09,1988 & 20.36 & MAY 29,1990 & 7.80 \\
\hline JUL 15 & 27.07 & MAY 16 & 26.85 & JUN 26 & 6.83 \\
\hline SEP 09 & 25.62 & JUL 05 & 44.16 & JUL 25 & 7.54 \\
\hline NOV 04 & 23.48 & SEP 14 & 48.10 & AUG 27 & 5.97 \\
\hline JAN 07, 1981 & 22.14 & NOV 29 & 33.99 & SEP 26 & 7.73 \\
\hline MAR 24 & 22.02 & JAN 06, 1989 & 31.55 & OCT 30 & 8.15 \\
\hline APR 15 & 21.95 & MAR 24 & 28.70 & NOV 27 & 8.07 \\
\hline MAY 19 & 22.93 & MAY 04 & 24.49 & JAN 04, 1991 & 8.26 \\
\hline JUL 08 & 38.21 & JUL 11 & 44.29 & MAR 08 & 6.98 \\
\hline AUG 20 & 44.00 & SEP 13 & 37.75 & & \\
\hline SEP 22 & 33.22 & NOV 21 & 32.69 & 15S 38E 22DDC1 & \\
\hline NOV 04 & 29.27 & JAN 23, 1990 & 29.80 & Altitude 4,760 & \\
\hline FEB 10, 1982 & 25.37 & FEB 28 & 28.87 & AUG 11, 1969 & 18.15 \\
\hline MAR 23 & 23.94 & MAR 13 & 27.44 & SEP 08 & 17.93 \\
\hline MAY 05 & 22.14 & APR 26 & 28.63 & OCT 10 & 19.22 \\
\hline JUN 15 & 19.29 & MAY 30 & 31.10 & NOV 13 & 21.02 \\
\hline JUL 27 & 24.20 & JUL 25 & 44.85 & DEC 04 & 21.74 \\
\hline SEP 08 & 25.70 & AUG 27 & 47.96 & JAN 08, 1970 & 22.64 \\
\hline OCT 27 & 21.83 & SEP 26 & 43.30 & FEB 11 & 23.10 \\
\hline DEC 07 & 20.90 & OCT 30 & 40.42 & MAR 09 & 23.38 \\
\hline JAN 13, 1983 & 20.26 & NOV 27 & 38.32 & SEP 29, 1977 & 22.34 \\
\hline MAR 02 & 19.84 & JAN 04, 1991 & 38.99 & MAR 21, 1978 & 25.30 \\
\hline MAY 25 & 17.73 & MAR 07 & 33.91 & JAN 04, 1990 & 25.21 \\
\hline JUL 07 & 15.85 & & & APR 18 & 24.19 \\
\hline SEP 20 & 17.17 & 14S 39E 08ADA1 & & 25 & 24.76 \\
\hline NOV 09 & 17.22 & Altitude 4,850 & & MAY 30 & 24.20 \\
\hline JAN 05, 1984 & 16.13 & AUG 11,1969 & 96.38 & JUN 26 & 22.29 \\
\hline MAR 28 & 16.70 & SEP 08 & 95.96 & JUL 24 & 20.78 \\
\hline MAY 30 & 14.28 & OCT 10 & 95.52 & AUG 27 & 21.76 \\
\hline JUL 18 & 15.56 & NOV 13 & 95.61 & SEP 26 & 22.91 \\
\hline SEP 05 & 14.85 & JAN 08, 1970 & 95.54 & OCT 30 & 23.97 \\
\hline NOV 15 & 15.41 & FEB 11 & 95.63 & NOV 27 & 24.63 \\
\hline JAN 04, 1985 & 15.27 & MAR 09 & 95.59 & JAN 04, 1991 & 25.21 \\
\hline MAR 20 & 16.29 & MAR 08, 1977 & 91.50 & MAR 07 & 25.85 \\
\hline MAY 02 & 15.04 & SEP 30 & 89.05 & & \\
\hline JUL 11 & 32.27 & MAR 21, 1978 & 92.14 & 15S 39E 15CAD1 & \\
\hline SEP 09 & 21.03 & FEB 28,1990 & 84.10 & Altitude 4,720 & \\
\hline NOV 20 & 17.90 & APR 10 & 84.55 & JUN 29, 1990 & 5.89 \\
\hline JAN 23, 1986 & 17.52 & 26 & 84.64 & JUL 25 & 5.31 \\
\hline MAR 05 & 15.34 & MAY 29 & 84.61 & AUG 27 & 2.76 \\
\hline MAY 21 & 12.41 & JUN 26 & 84.09 & SEP 26 & 6.88 \\
\hline JUL 01 & 22.27 & AUG 27 & 83.83 & OCT 31 & 7.12 \\
\hline SEP 24 & 16.75 & SEP 26 & 84.67 & NOV 27 & 7.16 \\
\hline NOV 07 & 15.94 & OCT 30 & 85.03 & JAN 04, 1991 & 7.64 \\
\hline JAN 28, 1987 & 15.75 & NOV 27 & 85.38 & MAR 08 & 6.20 \\
\hline MAR 11 & 15.80 & JAN 04, 1991 & 85.58 & & \\
\hline MAY 28 & 18.73 & MAR 08 & 86.48 & 15S 39E 23BBB 1 & \\
\hline JUL 09 & 43.05 & & & Alttude $4,725.4$ & \\
\hline SEP 22 & 24.00 & 14S 39E 29DAD1 & & AUG 11,1969 & 2.98 \\
\hline NOV 13 & 21.69 & Altitude 4,755 & & SEP 08 & 2.79 \\
\hline JAN 28, 1988 & 20.93 & APR 26,1990 & 7.66 & 23 & 3.64 \\
\hline
\end{tabular}


Table 3.-Water levels in selected wells-Continued

\begin{tabular}{|c|c|c|c|c|c|}
\hline Date & Water level & Date & Water level & Date & Water level \\
\hline \multicolumn{2}{|c|}{ 15S 39E 23BBB1-Continued } & \multicolumn{2}{|c|}{ 15S 39E 23BBB1-Continued } & \multicolumn{2}{|c|}{ 16S 39E 18CDA1-Continued } \\
\hline OCT 10, 1969 & 4.48 & MAR 23, 1981 & 1.56 & MAR 08, 1972 & 24.06 \\
\hline NOV 13 & 4.40 & APR 15 & 2.83 & SEP 27 & 40.86 \\
\hline DEC 04 & 4.75 & MAY 19 & 2.31 & MAR 28, 1973 & 25.65 \\
\hline JAN 08, 1970 & 4.91 & JUL 08 & 3.13 & SEP 26 & 42.00 \\
\hline FEB 11 & 2.03 & AUG 20 & 3.87 & MAR 20, 1974 & 27.06 \\
\hline MAR 09 & 2.57 & SEP 22 & 6.62 & SEP 23 & 51.69 \\
\hline 18 & 2.80 & NOV 04 & 5.62 & MAR 19, 1975 & 32.91 \\
\hline SEP 24 & 3.75 & MAR 23, 1982 & 1.33 & SEP 19 & 42.30 \\
\hline MAR 23, 1971 & .95 & MAY 05 & 2.51 & MAR 10, 1976 & 30.46 \\
\hline SEP 15 & 3.37 & JUN 15 & 2.44 & SEP 09 & 41.01 \\
\hline MAR 08, 1972 & 1.18 & JUL 27 & 3.25 & MAR 09, 1977 & 27.13 \\
\hline SEP 27 & 4.51 & SEP 08 & 3.63 & JUL 06 & 44.10 \\
\hline MAR 28, 1973 & .74 & OCT 27 & 2.52 & AUG 18 & 47.42 \\
\hline SEP 26 & 3.53 & DEC 07 & 1.74 & SEP 29 & 45.95 \\
\hline MAR 20, 1974 & 1.19 & MAR 02, 1983 & .83 & NOV 09 & 40.47 \\
\hline SEP $23^{\circ}$ & 5.02 & MAY 25 & 1.77 & DEC 21 & 37.05 \\
\hline MAR 19, 1975 & 1.28 & JUL 07 & 2.52 & FEB 09, 1978 & 33.25 \\
\hline SEP 19 & 3.85 & SEP 20 & 3.48 & MAR 21 & 30.83 \\
\hline MAR 10, 1976 & 2.20 & NOV 09 & 1.17 & APR 26 & 28.85 \\
\hline SEP 09 & 3.50 & JAN 05, 1984 & 1.08 & MAY 31 & 27.52 \\
\hline MAR 09, 1977 & 5.59 & MAR 28 & 1.04 & JUL 12 & 40.27 \\
\hline AUG 12 & 3.56 & MAY 30 & 3.20 & AUG 23 & 48.62 \\
\hline 18 & 3.96 & JUL 18 & 2.49 & SEP 26 & 46.02 \\
\hline SEP 20 & 4.54 & SEP 05 & 4.14 & NOV 08 & 40.00 \\
\hline 29 & 5.13 & & & DEC 20 & 35.49 \\
\hline NOV 09 & 6.18 & 15S 39E 34CBD1 & & JAN 24, 1979 & 32.91 \\
\hline DEC 21 & 4.19 & Altitude 4,650 & & MAR 07 & 30.86 \\
\hline FEB 08, 1978 & 1.42 & AUG 11, 1969 & 6.62 & APR 20 & 29.66 \\
\hline MAR 21 & 2.15 & SEP 08 & 6.98 & MAY 23 & 28.52 \\
\hline APR 26 & 2.13 & OCT 10 & 7.05 & JUL 25 & 48.13 \\
\hline MAY 31 & 3.39 & NOV 13 & 6.86 & SEP 12 & 48.55 \\
\hline JUL 12 & 2.68 & DEC 04 & 6.95 & OCT 24 & 46.19 \\
\hline AUG 23 & 3.67 & JAN 08, 1970 & 7.13 & DEC 12 & 40.26 \\
\hline SEP 26 & 4.45 & FEB 11 & 6.58 & FEB 07, 1980 & 35.58 \\
\hline NOV 08 & 5.64 & MAR 09 & 7.00 & MAR 26 & 31.49 \\
\hline DEC 20 & 5.00 & & & MAY 14 & 26.17 \\
\hline JAN 23, 1979 & 5.51 & 16S 39E 18CDA1 & & JUL 15 & 30.81 \\
\hline MAR 06 & 2.81 & Altitude $4,542.7$ & & SEP 09 & 38.72 \\
\hline APR 19 & 2.77 & AUG 11, 1969 & 26.61 & NOV 04 & 34.77 \\
\hline MAY 23 & 3.56 & SEP 08 & 32.34 & JAN 07, 1981 & 30.23 \\
\hline JUL 25 & 3.49 & 23 & 32.17 & MAR 24 & 25.27 \\
\hline SEP 12 & 4.72 & OCT 10 & 33.12 & APR 15 & 24.71 \\
\hline OCT 24 & 4.71 & NOV 13 & 30.29 & MAY 19 & 23.88 \\
\hline DEC 12 & 6.05 & DEC 04 & 27.91 & JUL 08 & 33.51 \\
\hline FEB 07, 1980 & 3.33 & JAN 08, 1970 & 27.68 & AUG 20 & 36.48 \\
\hline MAR 26 & 1.81 & FEB 11 & 23.50 & SEP 22 & 27.80 \\
\hline MAY 14 & 1.66 & MAR 03 & 22.43 & NOV 04 & 29.89 \\
\hline JUL 15 & 3.14 & 18 & 21.86 & MAY 05, 1982 & 24.16 \\
\hline SEP 10 & 3.37 & SEP 24 & 43.00 & JUN 15 & 21.93 \\
\hline NOV 04 & 4.75 & MAR 23, 1971 & 27.63 & JUL 27 & 28.57 \\
\hline JAN 07, 1981 & 4.08 & SEP 15 & 36.00 & SEP 08 & 33.67 \\
\hline
\end{tabular}


Table 3.-Water levels in selected wells-Continued

\begin{tabular}{|c|c|c|c|c|c|}
\hline Date & Water level & Date & Water level & Date & Water level \\
\hline \multicolumn{2}{|c|}{ 16S 39E 18CDA1-Continued } & \multicolumn{2}{|c|}{ 16S 39E 18CDA1-Continued } & \multicolumn{2}{|c|}{ 16S 40E 29CCB1-Continued } \\
\hline OCT 27, 1982 & 28.74 & NOV 27,1990 & 32.38 & MAR 25, 1970 & 15.32 \\
\hline MAR 02, 1983 & 27.00 & MAR 07 & 27.85 & 31 & 15.41 \\
\hline MAY 25 & 23.28 & & & APR 05 & 15.91 \\
\hline JUL 07 & 22.10 & 16S 40E 29CCB1 & & 10 & 16.60 \\
\hline SEP 20 & 28.23 & Altitude $4,504.9$ & & 15 & 16.89 \\
\hline NOV 09 & 25.93 & AUG 05, 1969 & 5.30 & 20 & 17.43 \\
\hline JAN 05, 1984 & 23.98 & 10 & 4.32 & 25 & 17.63 \\
\hline MAR 28 & 22.00 & 13 & 3.60 & 30 & 17.90 \\
\hline MAY 30 & 21.22 & 20 & 3.70 & MAY 05 & 17.88 \\
\hline JUL 18 & 26.96 & 25 & 4.98 & 10 & 17.96 \\
\hline SEP 05 & 26.86 & 31 & 6.15 & 15 & 18.07 \\
\hline NOV 15 & 24.23 & SEP 05 & 4.01 & 20 & 18.54 \\
\hline JAN 04, 1985 & 23.34 & 10 & 3.96 & 25 & 18.75 \\
\hline MAR 20 & 21.81 & 15 & 4.95 & 31 & 18.88 \\
\hline MAY 02 & 20.96 & 20 & 4.99 & JUN 05 & 19.00 \\
\hline JUL 11 & 29.19 & 25 & 5.95 & 10 & 19.05 \\
\hline SEP 09 & 33.40 & 30 & 6.73 & 15 & 19.19 \\
\hline NOV 20 & 26.25 & OCT 05 & 7.24 & 20 & 19.37 \\
\hline JAN 23, 1986 & 24.81 & 10 & 7.83 & 24 & 19.56 \\
\hline MAR 05 & 24.40 & 15 & 8.43 & 25 & 18.95 \\
\hline MAY 21 & 23.87 & 20 & 8.95 & 30 & 19.08 \\
\hline JUL 01 & 26.10 & 25 & 9.32 & JUL 05 & 15.30 \\
\hline SEP 24 & 28.16 & 31 & 9.33 & SEP 24 & 7.70 \\
\hline NOV 07 & 26.53 & NOV 05 & 10.57 & 25 & 7.83 \\
\hline JAN 28, 1987 & 24.60 & 10 & 11.20 & 30 & 8.38 \\
\hline MAR 11 & 24.32 & 15 & 11.87 & OCT 05 & 8.89 \\
\hline MAY 28 & 22.68 & 20 & 12.40 & 10 & 8.35 \\
\hline JUL 09 & 32.87 & 25 & 12.82 & 15 & 8.18 \\
\hline SEP 22 & 26.80 & 30 & 13.31 & 20 & 7.52 \\
\hline NOV 13 & 25.18 & DEC 05 & 13.86 & 25 & 5.83 \\
\hline JAN 28, 1988 & 23.75 & 10 & 14.50 & 31 & 6.67 \\
\hline MAR 09 & 23.08 & 15 & 15.28 & NOV 05 & 6.24 \\
\hline MAY 17 & 19.56 & 20 & 16.12 & 08 & 5.68 \\
\hline JUL 05 & 33.59 & 25 & 16.26 & 10 & 6.25 \\
\hline SEP 14 & 31.82 & 31 & 16.94 & 15 & 7.13 \\
\hline NOV 29 & 29.83 & JAN 05, 1970 & 17.30 & 20 & 7.38 \\
\hline JAN 06, 1989 & 29.84 & 10 & 17.71 & 25 & 6.63 \\
\hline MAR 24 & 25.96 & 15 & 18.26 & 30 & 7.18 \\
\hline MAY 04 & 25.10 & 20 & 16.70 & DEC 05 & 8.20 \\
\hline JUL 11 & 36.56 & 25 & 13.92 & 10 & 8.55 \\
\hline SEP 13 & 38.33 & 31 & 13.07 & MAR 23, 1971 & 3.90 \\
\hline NOV 21 & 30.83 & FEB 05, 1970 & 13.37 & 25 & 3.59 \\
\hline JAN 23, 1990 & 27.08 & 10 & 13.44 & 31 & 4.19 \\
\hline MAR 13 & 26.00 & 15 & 13.39 & APR 05 & 4.80 \\
\hline APR 18 & 21.90 & 20 & 13.08 & 10 & 5.45 \\
\hline 25 & 22.89 & 25 & 13.22 & 15 & 6.54 \\
\hline MAY 30 & 24.74 & 28 & 13.28 & 18 & 6.70 \\
\hline JUN 27 & 27.08 & MAR 05 & 13.50 & 20 & 6.57 \\
\hline JUL 24 & 36.35 & 10 & 13.72 & 25 & 5.88 \\
\hline SEP 26 & 38.19 & 15 & 13.74 & 26 & 3.57 \\
\hline OCT 29 & 33.97 & 20 & 13.91 & 30 & 3.89 \\
\hline
\end{tabular}


Table 3.-Water levels in selected wells-Continued

\begin{tabular}{|c|c|c|c|c|c|}
\hline Date & Water level & Date & Water level & Date & Water level \\
\hline \multicolumn{2}{|c|}{ 16S 40E 29CCB1-Continued } & \multicolumn{2}{|c|}{ 16S 40E 29CCB1-Continued } & \multicolumn{2}{|c|}{ 16S 40E 29CCB1-Continued } \\
\hline MAY 05, 1971 & 4.83 & NOV 30,1978 & 16.72 & JUL 20, 1979 & 7.69 \\
\hline 10 & 5.85 & DEC 05 & 15.90 & 25 & 4.17 \\
\hline 15 & 4.70 & 10 & 15.74 & SEP 15 & 6.41 \\
\hline 20 & 4.06 & 11 & 15.72 & 20 & 7.16 \\
\hline 25 & 4.00 & 15 & 15.96 & 25 & 7.84 \\
\hline 31 & 3.63 & 20 & 16.32 & 30 & 8.71 \\
\hline JUN 05 & 3.33 & 25 & 16.65 & OCT 05 & 9.86 \\
\hline 10 & 3.20 & 31 & 17.04 & 10 & 11.04 \\
\hline 15 & 3.01 & JAN 01, 1979 & 17.02 & 15 & 12.26 \\
\hline 20 & 2.68 & 05 & 17.33 & 20 & 12.90 \\
\hline 25 & 2.18 & 10 & 17.83 & 25 & 13.27 \\
\hline 27 & 1.97 & 15 & 18.15 & 31 & 14.03 \\
\hline 30 & 4.30 & 20 & 18.49 & DEC 12 & 19.25 \\
\hline JUL 06 & 6.36 & 23 & 18.73 & 15 & 19.36 \\
\hline 09 & 5.87 & 25 & 18.65 & 20 & 19.36 \\
\hline 18 & 6.51 & 31 & 19.10 & 25 & 19.35 \\
\hline 20 & 6.14 & FEB 03 & 19.34 & 31 & 19.35 \\
\hline 25 & 5.20 & 05 & 19.34 & JAN 05, 1980 & 19.35 \\
\hline 27 & 5.83 & 10 & 19.34 & 10 & 17.82 \\
\hline AUG 05 & 3.93 & 15 & 18.64 & 15 & 8.27 \\
\hline 10 & 5.52 & 20 & 18.47 & 20 & 10.77 \\
\hline 15 & 5.61 & 25 & 18.61 & 25 & 12.03 \\
\hline 20 & 5.63 & 28 & 18.78 & 31 & 12.98 \\
\hline 25 & 5.49 & MAR 05 & 18.83 & FEB 05 & 13.56 \\
\hline 31 & 5.05 & 10 & 18.40 & 10 & 14.16 \\
\hline SEP 15 & 6.58 & 15 & 17.01 & 15 & 12.45 \\
\hline MAR 08, 1972 & 6.07 & 20 & 13.43 & 20 & 8.88 \\
\hline SEP 27 & 9.46 & 25 & 9.44 & 25 & 8.90 \\
\hline MAR 28, 1973 & 10.48 & 31 & 7.50 & 29 & 8.91 \\
\hline SEP 26 & 7.57 & APR 05 & 7.79 & MAR 05 & 7.95 \\
\hline MAR 20, 1974 & 3.86 & 10 & 7.74 & 07 & 7.70 \\
\hline SEP 23 & 9.27 & 15 & 7.93 & 26 & 8.34 \\
\hline MAR 19, 1975 & 10.49 & 20 & 8.24 & 31 & 7.87 \\
\hline SEP 19 & 8.50 & 25 & 8.77 & APR 03 & 7.16 \\
\hline MAR 10, 1976 & 15.08 & 30 & 9.27 & 05 & 7.16 \\
\hline SEP 09 & 7.93 & MAY 05 & 9.69 & 10 & 7.37 \\
\hline MAR 09, 1977 & 23.37 & 10 & 10.14 & 15 & 7.77 \\
\hline JUL 06 & 3.44 & 15 & 10.46 & 20 & 8.20 \\
\hline AUG 18 & 6.70 & 20 & 11.03 & 25 & 8.42 \\
\hline SEP 29 & 10.66 & 25 & 11.44 & 30 & 8.83 \\
\hline NOV 09 & 16.47 & 31 & 12.16 & MAY 05 & 9.27 \\
\hline DEC 21 & 16.46 & JUN 05 & 12.62 & 10 & 9.37 \\
\hline FEB 08, 1978 & 13.47 & 10 & 13.14 & 14 & 7.93 \\
\hline MAR 21 & 9.37 & 15 & 13.62 & JUL 15 & 9.03 \\
\hline APR 26 & 8.00 & 20 & 14.11 & 20 & 9.95 \\
\hline MAY 31 & 11.00 & 25 & 14.55 & 25 & 8.87 \\
\hline JUL 12 & 2.18 & 29 & 14.91 & 31 & 8.55 \\
\hline AUG 23 & 3.92 & 30 & 9.66 & AUG 05 & 9.00 \\
\hline SEP 26 & 8.69 & JUL 05 & 5.20 & 10 & 9.67 \\
\hline NOV 08 & 14.85 & 10 & 7.85 & 15 & 10.52 \\
\hline 25 & 16.50 & 15 & 8.91 & 20 & 11.31 \\
\hline
\end{tabular}


Table 3.-Water levels in selected wells-Continued

\begin{tabular}{|c|c|c|c|c|c|}
\hline Date & Water level & Date & Water level & Date & Water level \\
\hline \multicolumn{2}{|c|}{ 16S 40E 29CCB1-Continued } & \multicolumn{2}{|c|}{ 16S 40E 29CCB1-Continued } & \multicolumn{2}{|c|}{ 16S 40E 29CCB1-Continued } \\
\hline AUG 25, 1980 & 11.97 & SEP 10, 1981 & 14.84 & MAY 20, 1982 & 6.37 \\
\hline 31 & 12.89 & 15 & 16.11 & 25 & 6.69 \\
\hline SEP 05 & 13.70 & 25 & 17.83 & 31 & 7.07 \\
\hline 06 & 13.88 & 30 & 18.58 & JUN 05 & 7.53 \\
\hline 10 & 13.77 & OCT 05 & 19.23 & 10 & 8.00 \\
\hline 11 & 13.70 & 10 & 19.65 & 15 & 8.69 \\
\hline 15 & 14.10 & 15 & 19.76 & 20 & 9.69 \\
\hline 20 & 14.80 & 20 & 20.09 & JUL 31 & 10.39 \\
\hline 25 & 15.54 & 25 & 20.48 & AUG 05 & 11.36 \\
\hline 30 & 16.27 & 31 & 21.03 & 10 & 11.93 \\
\hline ОСТ 05 & 16.84 & NOV 04 & 21.25 & 15 & 11.45 \\
\hline 10 & 17.52 & 05 & 21.33 & 20 & 12.14 \\
\hline 15 & 17.94 & 10 & 21.64 & 25 & 12.44 \\
\hline 20 & 18.44 & 15 & 21.84 & 31 & 13.09 \\
\hline 25 & 18.76 & 20 & 22.15 & SEP 05 & 13.74 \\
\hline 31 & 19.26 & 25 & 22.24 & 10 & 13.91 \\
\hline NOV 03 & 19.45 & 30 & 22.43 & 15 & 14.18 \\
\hline 04 & 19.48 & DEC 05 & 22.53 & 20 & 14.84 \\
\hline JAN 07, 1981 & 22.83 & 10 & 22.67 & 25 & 15.56 \\
\hline MAR 24 & 18.74 & 11 & 22.70 & 30 & 15.16 \\
\hline 25 & 18.52 & 15 & 22.48 & OCT 01 & 15.12 \\
\hline 31 & 17.08 & 20 & 22.39 & 05 & 14.59 \\
\hline APR 05 & 16.42 & 25 & 22.03 & 10 & 14.29 \\
\hline 10 & 16.29 & 31 & 21.34 & 15 & 14.27 \\
\hline 15 & 16.52 & JAN 05, 1982 & 20.49 & 20 & 14.37 \\
\hline 20 & 16.72 & 10 & 20.12 & 25 & 14.56 \\
\hline 25 & 16.88 & 15 & 19.70 & 31 & 14.57 \\
\hline 30 & 16.13 & 20 & 19.68 & NOV 05 & 14.38 \\
\hline MAY 05 & 15.91 & 25 & 19.76 & 10 & 14.25 \\
\hline 10 & 15.98 & 31 & 19.62 & 15 & 14.22 \\
\hline 15 & 16.20 & FEB 05 & 19.64 & 20 & 14.05 \\
\hline 20 & 16.49 & 10 & 19.53 & 25 & 13.58 \\
\hline 25 & 16.35 & 15 & 19.66 & 30 & 13.28 \\
\hline 31 & 15.28 & 20 & 17.51 & DEC 05 & 13.47 \\
\hline JUN 03 & 15.16 & 25 & 13.40 & 10 & 13.29 \\
\hline 05 & 15.23 & 28 & 12.69 & 15 & 13.22 \\
\hline 10 & 15.41 & MAR 05 & 9.32 & 20 & 13.22 \\
\hline 15 & 15.90 & 10 & 7.64 & 25 & 13.23 \\
\hline JUL 10 & 15.03 & 15 & 6.28 & 31 & 12.97 \\
\hline 15 & 10.35 & 20 & 4.85 & JAN 05, 1983 & 13.05 \\
\hline 20 & 11.73 & 25 & 5.27 & 10 & 12.74 \\
\hline 25 & 12.72 & 31 & 5.37 & 13 & 12.55 \\
\hline 31 & 13.77 & APR 05 & 4.83 & 15 & 12.51 \\
\hline AUG 02 & 8.30 & 10 & 4.96 & 20 & 12.63 \\
\hline 05 & 8.87 & 12 & 3.01 & 25 & 12.88 \\
\hline 10 & 9.69 & 15 & 4.03 & 31 & 12.56 \\
\hline 15 & 9.99 & 20 & 4.76 & FEB 05 & 12.65 \\
\hline 20 & 10.93 & 22 & 4.98 & 10 & 12.69 \\
\hline 25 & 11.81 & MAY 05 & 5.08 & 15 & 12.11 \\
\hline 31 & 12.94 & 10 & 5.72 & 20 & 11.11 \\
\hline SEP 05 & 13.85 & 15 & 6.03 & 25 & 10.40 \\
\hline
\end{tabular}


Table 3.-Water levels in selected wells-Continued

\begin{tabular}{|c|c|c|c|c|c|}
\hline Date & Water level & Date & Water level & Date & Water level \\
\hline \multicolumn{2}{|c|}{ 16S 40E 29CCB1-Continued } & \multicolumn{2}{|c|}{ 16S 40E 29CCB1-Continued } & \multicolumn{2}{|c|}{ 16S 40E 29CCB1-Continued } \\
\hline FEB 28, 1983 & 9.62 & NOV 05,1983 & 10.91 & AUG 20, 1984 & 5.39 \\
\hline MAR 05 & 7.80 & 10 & 10.86 & 25 & 5.66 \\
\hline 10 & 7.17 & 15 & 10.45 & 31 & 6.21 \\
\hline 15 & 6.02 & 20 & 10.01 & SEP 05 & 6.71 \\
\hline 20 & 5.65 & 25 & 9.66 & 10 & 7.11 \\
\hline 25 & 4.30 & 30 & 8.96 & 15 & 7.64 \\
\hline 31 & 4.37 & DEC 05 & 8.56 & 20 & 8.24 \\
\hline APR 05 & 4.23 & 10 & 7.59 & 25 & 8.09 \\
\hline 10 & 4.53 & 15 & 6.75 & 30 & 8.26 \\
\hline 15 & 4.06 & 20 & 6.44 & OCT 05 & 8.62 \\
\hline 20 & 4.58 & 25 & 6.24 & 10 & 9.02 \\
\hline 25 & 4.83 & 31 & 6.15 & 15 & 8.89 \\
\hline 30 & 4.38 & JAN 05, 1984 & 6.06 & 20 & 9.03 \\
\hline MAY 05 & 2.68 & 10 & 5.94 & 25 & 9.15 \\
\hline 10 & 3.68 & 15 & 5.88 & 31 & 9.15 \\
\hline 15 & 3.33 & 20 & 5.84 & NOV 05 & 9.06 \\
\hline 19 & 2.00 & 25 & 5.94 & 10 & 8.57 \\
\hline 20 & 2.67 & 31 & 5.94 & 15 & 8.00 \\
\hline 25 & 3.89 & FEB 05 & 6.06 & 20 & 8.08 \\
\hline 31 & 4.74 & 10 & 6.18 & 25 & 8.28 \\
\hline JUN 05 & 5.29 & 15 & 5.54 & 30 & 8.34 \\
\hline 10 & 5.83 & 20 & 5.58 & DEC 05 & 8.46 \\
\hline 15 & 6.13 & 25 & 5.60 & 10 & 8.61 \\
\hline 20 & 6.50 & 29 & 5.61 & 15 & 8.83 \\
\hline 25 & 6.99 & MAR 05 & 5.72 & 20 & 9.06 \\
\hline 30 & 7.46 & 10 & 5.47 & 25 & 9.34 \\
\hline JUL 05 & 7.24 & 15 & 3.44 & 31 & 9.65 \\
\hline 10 & 7.69 & 20 & 1.88 & JAN 15, 1985 & 10.43 \\
\hline 15 & 8.26 & 25 & 1.84 & 20 & 10.68 \\
\hline 20 & 9.04 & 26 & 1.32 & 25 & 10.94 \\
\hline 25 & 9.85 & 31 & .94 & 31 & 11.30 \\
\hline 31 & 10.65 & APR 01 & .57 & FEB 05 & 11.63 \\
\hline AUG 05 & 11.36 & 05 & 1.99 & 10 & 11.94 \\
\hline 10 & 10.55 & 10 & 1.76 & 15 & 12.23 \\
\hline 15 & 10.35 & MAY 30 & 5.26 & 20 & 12.40 \\
\hline 20 & 7.83 & 31 & 5.33 & 25 & 12.71 \\
\hline 25 & 8.25 & JUN 05 & 5.61 & 28 & 12.84 \\
\hline 31 & 9.03 & 10 & 5.13 & MAR 05 & 13.01 \\
\hline SEP 05 & 9.55 & 15 & 5.60 & 10 & 13.24 \\
\hline 10 & 10.13 & 20 & 5.93 & 15 & 13.39 \\
\hline 15 & 10.74 & 25 & 6.33 & 18 & 13.53 \\
\hline 20 & 11.36 & 30 & 6.79 & 20 & 13.46 \\
\hline 25 & 11.92 & JUL 05 & 7.29 & 25 & 13.20 \\
\hline 30 & 11.87 & 10 & 7.90 & 31 & 11.46 \\
\hline OCT 01 & 11.94 & 15 & 8.51 & APR 05 & 6.64 \\
\hline 05 & 10.81 & 20 & 9.23 & 08 & 1.84 \\
\hline 10 & 10.61 & 25 & 9.63 & 10 & 3.01 \\
\hline 15 & 10.43 & 31 & 5.04 & 15 & 3.78 \\
\hline 20 & 10.45 & AUG 05 & 4.75 & 20 & 4.44 \\
\hline 25 & 10.59 & 10 & 5.77 & 25 & 4.71 \\
\hline 31 & 10.77 & 15 & 6.49 & 30 & 5.10 \\
\hline
\end{tabular}


Table 3.-Water levels in selected wells-Continued

\begin{tabular}{|c|c|c|c|c|c|}
\hline Date & Water level & Date & Water level & Date & Water level \\
\hline \multicolumn{2}{|c|}{ 16S 40E 29CCB1-Continued } & \multicolumn{2}{|c|}{ 16S 40E 29CCB1-Continued } & \multicolumn{2}{|c|}{ 16S 40E 29CCB1-Continued } \\
\hline MAY 05, 1985 & 5.67 & JAN 15, 1986 & 9.68 & SEP 25,1986 & 7.92 \\
\hline 10 & 6.07 & 20 & 9.71 & 30 & 7.44 \\
\hline 15 & 6.33 & 25 & 9.28 & OCT 05 & 7.47 \\
\hline 20 & 6.68 & 31 & 9.28 & 10 & 7.79 \\
\hline 25 & 7.07 & FEB 05 & 8.35 & 15 & 8.18 \\
\hline 31 & 7.67 & 10 & 8.16 & 20 & 8.45 \\
\hline JUN 05 & 8.31 & 15 & 7.32 & 25 & 8.73 \\
\hline 10 & 9.08 & 19 & .60 & 31 & 9.20 \\
\hline 15 & 9.83 & 20 & 1.09 & NOV 07 & 9.33 \\
\hline 20 & 10.13 & 25 & 2.29 & 10 & 9.51 \\
\hline 25 & 8.65 & 28 & 2.68 & 15 & 9.80 \\
\hline 30 & 7.14 & MAR 05 & 3.16 & 20 & 10.06 \\
\hline JUL 05 & 4.75 & 10 & 2.34 & 25 & 10.31 \\
\hline 10 & 3.09 & 15 & 2.69 & 30 & 10.56 \\
\hline 15 & 4.27 & 20 & 2.92 & DEC 05 & 10.84 \\
\hline 20 & 5.66 & 25 & 3.52 & 10 & 11.03 \\
\hline 25 & 6.37 & 31 & 4.08 & 15 & 11.15 \\
\hline 31 & 7.14 & APR 05 & 3.01 & 20 & 11.54 \\
\hline AUG 05 & 7.62 & 10 & 3.68 & 25 & 11.81 \\
\hline 10 & 6.80 & 15 & 2.71 & 31 & 12.05 \\
\hline 15 & 2.81 & 20 & 3.27 & JAN 05, 1987 & 12.44 \\
\hline 20 & 3.49 & 25 & 2.48 & 10 & 12.64 \\
\hline 25 & 5.14 & 30 & 2.29 & 15 & 12.93 \\
\hline 31 & 5.92 & MAY 05 & 3.09 & 20 & 13.31 \\
\hline SEP 05 & 6.38 & 10 & 2.29 & 25 & 13.60 \\
\hline 10 & 6.71 & 15 & 3.04 & 31 & 13.86 \\
\hline 15 & 7.27 & 20 & 3.83 & FEB 03 & 14.02 \\
\hline 20 & 7.71 & 25 & 4.29 & 05 & 11.52 \\
\hline 25 & 8.16 & 31 & 4.91 & 10 & 12.00 \\
\hline 30 & 8.57 & JUN 05 & 5.49 & 15 & 9.51 \\
\hline ОСТ 05 & 9.01 & 10 & 5.95 & 20 & 10.17 \\
\hline 10 & 9.38 & 15 & 6.36 & 25 & 10.39 \\
\hline 15 & 9.69 & 20 & 6.75 & 28 & 10.47 \\
\hline 20 & 10.12 & 25 & 7.03 & MAR 05 & 10.50 \\
\hline 25 & 10.31 & 30 & 3.45 & 10 & 10.38 \\
\hline 31 & 10.86 & JUL 05 & 2.86 & 15 & 10.26 \\
\hline NOV 05 & 11.25 & 10 & 4.25 & 20 & 10.30 \\
\hline 10 & 11.50 & 15 & 5.02 & 25 & 10.25 \\
\hline 14 & 11.77 & 20 & 5.72 & 31 & 10.38 \\
\hline 15 & 11.75 & 25 & 6.14 & APR 05 & 10.64 \\
\hline 20 & 11.61 & 31 & 6.62 & 10 & 10.74 \\
\hline 25 & 11.26 & AUG 05 & 7.15 & 15 & 10.89 \\
\hline 30 & 9.71 & 10 & 7.77 & 20 & 11.16 \\
\hline DEC 05 & 9.12 & 15 & 6.88 & 25 & 11.34 \\
\hline 10 & 8.84 & 20 & 2.46 & 30 & 11.67 \\
\hline 15 & 8.75 & 25 & 4.71 & MAY 05 & 11.92 \\
\hline 20 & 8.86 & 31 & 5.38 & 10 & 12.17 \\
\hline 25 & 9.05 & SEP 05 & 6.17 & 15 & 12.46 \\
\hline 31 & 9.32 & 10 & 6.71 & 20 & 12.49 \\
\hline JAN 05, 1986 & 9.45 & 15 & 7.24 & 25 & 12.47 \\
\hline 10 & 9.55 & 20 & 7.72 & 31 & 12.44 \\
\hline
\end{tabular}


Table 3.-Water levels in selected wells-Continued

\begin{tabular}{|c|c|c|c|c|c|}
\hline Date & Water level & Date & Water level & Date & Water level \\
\hline \multicolumn{2}{|c|}{ 16S 40E 29CCB1-Continued } & \multicolumn{2}{|c|}{ 16S 40E 29CCB1-Continued } & \multicolumn{2}{|c|}{ 16S 40E 29CCB1-Continued } \\
\hline JUN 05, 1987 & 12.63 & FEB 15, 1988 & 19.34 & NOV 05, 1988 & 12.61 \\
\hline 10 & 12.87 & 20 & 19.61 & 10 & 12.80 \\
\hline 15 & 13.29 & 22 & 19.79 & 15 & 13.14 \\
\hline 20 & 13.75 & 25 & 19.74 & 20 & 13.46 \\
\hline 25 & 13.94 & 29 & 18.88 & 25 & 13.67 \\
\hline 30 & 4.11 & MAR 05 & 14.67 & 30 & 13.50 \\
\hline JUL 05 & 7.21 & 10 & 14.04 & DEC 05 & 13.47 \\
\hline 10 & 4.39 & 15 & 13.95 & 10 & 13.88 \\
\hline 15 & 6.95 & 20 & 14.04 & 15 & 14.28 \\
\hline 20 & 7.91 & 25 & 14.24 & 20 & 14.69 \\
\hline 25 & 8.59 & 31 & 14.48 & 25 & 15.10 \\
\hline 31 & 9.69 & APR 05 & 14.70 & 30 & 15.59 \\
\hline AUG 05 & 9.69 & 10 & 14.91 & 31 & 15.64 \\
\hline 10 & 7.36 & 15 & 15.18 & JAN 05, 1989 & 16.10 \\
\hline 15 & 5.78 & 20 & 15.42 & 10 & 16.67 \\
\hline 20 & 2.65 & 25 & 15.52 & 15 & 17.06 \\
\hline 22 & .72 & 30 & 15.56 & 20 & 17.44 \\
\hline 25 & 3.66 & MAY 05 & 15.48 & 25 & 17.83 \\
\hline 31 & 5.27 & 10 & 15.59 & 31 & 18.67 \\
\hline SEP 05 & 6.21 & 15 & 15.80 & FEB 05 & 18.86 \\
\hline 10 & 6.69 & 20 & 15.95 & 10 & 19.06 \\
\hline 15 & 7.23 & 25 & 15.99 & 15 & 19.11 \\
\hline 20 & 7.85 & 31 & 16.25 & 20 & 19.19 \\
\hline 25 & 8.59 & JUN 05 & 4.81 & 25 & 19.47 \\
\hline 30 & 9.27 & 10 & 7.12 & 28 & 19.27 \\
\hline OCT 05 & 9.93 & 15 & 8.62 & MAR 05 & 17.42 \\
\hline 10 & 10.48 & 20 & 9.84 & 10 & 13.34 \\
\hline 15 & 10.87 & 25 & 7.16 & 15 & 9.10 \\
\hline 20 & 11.45 & 30 & 7.42 & 20 & 5.50 \\
\hline 25 & 12.07 & JUL 05 & 8.55 & 25 & 5.26 \\
\hline 31 & 12.67 & 10 & 9.74 & 31 & 5.58 \\
\hline NOV 05 & 13.11 & 15 & 10.96 & APR 05 & 6.20 \\
\hline 10 & 13.58 & 20 & 7.21 & 10 & 6.61 \\
\hline 15 & 13.84 & 25 & 9.80 & 15 & 6.95 \\
\hline 20 & 14.07 & 31 & 11.21 & 20 & 7.23 \\
\hline 25 & 14.34 & AUG 05 & 3.04 & 25 & 7.53 \\
\hline 30 & 14.64 & 10 & 6.36 & 30 & 7.82 \\
\hline DEC 05 & 14.88 & 15 & 7.39 & MAY 05 & 8.17 \\
\hline 10 & 15.11 & 20 & 7.20 & 10 & 8.37 \\
\hline 15 & 15.33 & 25 & 7.47 & 15 & 8.73 \\
\hline 20 & 15.53 & 26 & 6.50 & 20 & 9.19 \\
\hline 25 & 15.95 & SEP 15 & 8.40 & 25 & 9.66 \\
\hline 31 & 16.28 & 20 & 9.07 & 31 & 10.06 \\
\hline JAN 05, 1988 & 16.50 & 25 & 9.91 & JUN 05 & 10.56 \\
\hline 10 & 16.84 & 30 & 10.52 & 10 & 11.08 \\
\hline 15 & 17.21 & OCT 05 & 11.02 & 15 & 11.62 \\
\hline 20 & 17.65 & 10 & 11.42 & 20 & 2.85 \\
\hline 25 & 18.48 & 15 & 12.36 & 25 & 4.45 \\
\hline 31 & 18.21 & 20 & 12.00 & 30 & 6.35 \\
\hline FEB 05 & 18.59 & 25 & 12.42 & JUL 05 & 7.18 \\
\hline 10 & 19.05 & 31 & 12.45 & 10 & 8.10 \\
\hline
\end{tabular}


Table 3.-Water levels in selected wells-Continued

\begin{tabular}{|c|c|}
\hline Date & Water level \\
\hline \multicolumn{2}{|c|}{ 16S 40E 29CCB1-Continued } \\
\hline JUL 15, 1989 & 9.08 \\
\hline 20 & 9.61 \\
\hline 25 & 10.30 \\
\hline 31 & 11.46 \\
\hline AUG 05 & 11.81 \\
\hline 10 & 12.85 \\
\hline 15 & 13.34 \\
\hline 20 & 13.19 \\
\hline 25 & 6.52 \\
\hline 31 & 8.29 \\
\hline SEP 05 & 9.70 \\
\hline 10 & 10.75 \\
\hline 15 & 11.54 \\
\hline 20 & 12.55 \\
\hline 25 & 13.31 \\
\hline 30 & 14.34 \\
\hline ОСТ 05 & 15.40 \\
\hline 10 & 16.36 \\
\hline 15 & 17.12 \\
\hline 20 & 17.37 \\
\hline 25 & 17.91 \\
\hline 31 & 18.30 \\
\hline NOV 05 & 18.54 \\
\hline 10 & 19.29 \\
\hline 15 & 19.85 \\
\hline 20 & 20.00 \\
\hline 25 & 20.29 \\
\hline 30 & 20.22 \\
\hline DEC 05 & 20.72 \\
\hline 10 & 20.74 \\
\hline 15 & 21.73 \\
\hline 20 & 21.94 \\
\hline 25 & 21.96 \\
\hline 31 & 23.63 \\
\hline
\end{tabular}

\begin{tabular}{rr}
\hline Date & Water level \\
\hline 16S 40 E 29CCB1-Continued \\
JAN 05, 1990 & 23.84 \\
10 & 24.22 \\
15 & 22.97 \\
20 & 23.74 \\
25 & 24.91 \\
31 & 24.98 \\
FEB 05 & 25.33 \\
10 & 25.54 \\
15 & 25.41 \\
20 & 25.76 \\
21 & 25.78 \\
22 & 25.78 \\
25 & 21.48 \\
28 & 19.49 \\
MAR 05 & 20.76 \\
10 & 20.73 \\
15 & 20.21 \\
20 & 20.81 \\
25 & 19.98 \\
31 & 20.83 \\
APR 04 & 20.73 \\
10 & 20.98 \\
15 & 21.78 \\
20 & 22.33 \\
25 & 22.60 \\
26 & 22.75 \\
30 & 23.41 \\
MAY 05 & 23.63 \\
10 & 24.22 \\
15 & 23.93 \\
20 & 24.05 \\
24 & 19.98 \\
29 & 8.83 \\
JUN 26 & 6.37 \\
& \\
\hline & \\
& \\
&
\end{tabular}

\begin{tabular}{cc}
\hline Date & Water level \\
\hline 16S 40E 29CCB1-Continued \\
JUL 20, 1990 & 6.29 \\
25 & 7.89 \\
31 & 9.77 \\
AUG 05 & 5.91 \\
06 & 3.73 \\
10 & 6.50 \\
15 & 7.64 \\
20 & 8.67 \\
25 & 9.80 \\
27 & 10.22 \\
31 & 10.85 \\
SEP 05 & 11.87 \\
10 & 13.01 \\
15 & 14.53 \\
20 & 15.90 \\
25 & 17.37 \\
26 & 17.54 \\
30 & 18.42 \\
OCT 05 & 19.51 \\
10 & 20.58 \\
15 & 21.72 \\
20 & 22.83 \\
25 & 23.61 \\
31 & 24.17 \\
NOV 01 & 24.39 \\
05 & 24.64 \\
11 & 25.02 \\
15 & 25.35 \\
20 & 25.27 \\
25 & 25.72 \\
27 & 25.71 \\
JAN 04, 1991 & 27.09 \\
17 & 27.52 \\
MAR 08 & 22.44 \\
& \\
\hline & \\
\hline
\end{tabular}




\section{Table 4.-Discharge of selected flowing wells}

Location: See page 2 for an explanation of the numbering systems for hydrologic-data sites.

Discharge: $\mathrm{gal} / \mathrm{min}$, gallons per minute.

\begin{tabular}{|c|c|c|c|c|c|}
\hline Location & Date & $\begin{array}{c}\text { Discharge } \\
\text { (gal/min) }\end{array}$ & Location & Date & $\begin{array}{c}\text { Discharge } \\
\text { (gal/min) }\end{array}$ \\
\hline \multirow[t]{8}{*}{$(A-11-1) 18$ baa -1} & $03-01-90$ & 30.0 & $(B-12-1) 8 c d b-2$ & $08-27-90$ & 1.2 \\
\hline & $06-28-90$ & 30.0 & & $09-25-90$ & 1.2 \\
\hline & $07-26-90$ & 30.0 & & $10-29-90$ & 1.2 \\
\hline & $09-27-90$ & 30.0 & & $11-30-90$ & 1.2 \\
\hline & $10-29-90$ & 33.0 & & $01-16-91$ & 1.2 \\
\hline & $11-28-90$ & 37.5 & & $03-09-91$ & 1.2 \\
\hline & $01-17-91$ & 37.5 & $(B-12-1) 15 a d c-1$ & $04-19-90$ & 1.5 \\
\hline & $03-08-91$ & 30.0 & & $05-30-90$ & 2.0 \\
\hline \multirow[t]{10}{*}{$(A-12-1) 17$ daa-1 } & $04-26-90$ & 4.6 & & $06-28-90$ & 1.7 \\
\hline & $05-29-90$ & 4.0 & & $07-24-90$ & 1.9 \\
\hline & $06-28-90$ & 3.8 & & $08-27-90$ & 2.0 \\
\hline & $07-25-90$ & 3.8 & & $09-25-90$ & 1.9 \\
\hline & $09-26-90$ & 3.3 & & $10-29-90$ & 1.7 \\
\hline & $10-31-90$ & 3.5 & & $11-29-90$ & 1.6 \\
\hline & $11-20-90$ & 3.8 & & $01-17-91$ & 1.7 \\
\hline & $12-20-90$ & 3.5 & & $03-17-91$ & 1.6 \\
\hline & $01-17-91$ & 3.5 & $(B-12-1) 15 a d c-2$ & $10-29-90$ & 3.8 \\
\hline & $03-09-91$ & 3.3 & & $11-29-90$ & 3.3 \\
\hline \multirow[t]{13}{*}{$(A-14-1) 22 b a d-1$} & $03-11-80$ & 6.0 & & $01-17-91$ & 4.0 \\
\hline & $09-18-80$ & 6.0 & & $03-07-91$ & 4.0 \\
\hline & $03-04-81$ & 4.3 & $(B-12-1) 26 c c a-1$ & $03-01-90$ & .7 \\
\hline & $09-22-81$ & 5 & & $04-25-90$ & .8 \\
\hline & $03-08-82$ & 7.0 & & $08-27-90$ & .5 \\
\hline & $09-09-82$ & 10 & & $09-25-90$ & .6 \\
\hline & $09-18-89$ & 1.3 & & $10-29-90$ & .6 \\
\hline & $03-01-90$ & .5 & & $01-16-91$ & .6 \\
\hline & $04-26-90$ & 6.0 & $(B-13-1) 30$ acc-1 & $03-01-73$ & .7 \\
\hline & $04-27-90$ & 6.0 & & $09-12-73$ & .7 \\
\hline & $05-30-90$ & 8.6 & & $03-13-74$ & .6 \\
\hline & $06-28-90$ & 10.0 & & $09-11-74$ & .6 \\
\hline & $07-24-90$ & 3.5 & & $03-10-75$ & .6 \\
\hline \multirow[t]{11}{*}{$(B-11-1) 9 c d b-1$} & $03-01-90$ & .7 & & $10-14-75$ & .6 \\
\hline & $04-25-90$ & .5 & & $03-15-76$ & .7 \\
\hline & $05-29-90$ & .6 & & $09-14-76$ & .7 \\
\hline & $06-28-90$ & .6 & & $03-08-77$ & .6 \\
\hline & $07-24-90$ & .6 & & $09-28-77$ & .8 \\
\hline & $08-27-90$ & .5 & & $03-09-78$ & .7 \\
\hline & $09-25-90$ & .5 & & $09-19-78$ & .7 \\
\hline & $10-29-90$ & .6 & & $03-12-79$ & .7 \\
\hline & $11-28-90$ & .6 & & $09-19-79$ & .6 \\
\hline & $01-17-91$ & .6 & & $03-10-80$ & .6 \\
\hline & $03-06-91$ & .6 & & $09-17-80$ & .6 \\
\hline \multirow[t]{5}{*}{$(B-12-1) 8 c d b-2$} & $03-01-90$ & 1.1 & & $03-02-81$ & .7 \\
\hline & $04-25-90$ & 1.1 & & $09-17-81$ & .8 \\
\hline & $05-29-90$ & 1.1 & & $03-08-82$ & .7 \\
\hline & $06-28-90$ & 1.3 & & $03-01-90$ & .5 \\
\hline & $07-24-90$ & 1.4 & & $04-25-90$ & .5 \\
\hline
\end{tabular}


Table 4.-Discharge of selected flowing wells-Continued

\begin{tabular}{lcc}
\hline Location & Date & $\begin{array}{c}\text { Discharge } \\
\text { (gal/min) }\end{array}$ \\
\hline (B-13-1)30acc-1 & $05-29-90$ & 0.3 \\
& $06-28-90$ & .4 \\
& $07-24-90$ & .4 \\
& $08-27-90$ & .4 \\
& $09-25-90$ & .4 \\
\hline
\end{tabular}

\begin{tabular}{lcc}
\hline Location & Date & $\begin{array}{c}\text { Discharge } \\
\text { (gal/min) }\end{array}$ \\
\hline (B-13-1)30acc-1 & $10-29-90$ & 0.4 \\
& $11-29-90$ & .4 \\
& $01-16-91$ & .4 \\
& $03-07-91$ & .4 \\
& & \\
\hline
\end{tabular}




\section{Table 5.-Temperature and specific conductance of water from selected wells}

Location: See page 2 for an explanation of the numbering systems for hydrologic-data sites.

Water temperature: In degrees Celsius.

Specific conductance: In microsiemens per centimeter at 25 degrees Celsius; - indicates no data available.

\begin{tabular}{|c|c|c|c|}
\hline Location & Date & $\begin{array}{c}\text { Water } \\
\text { temperature }\end{array}$ & $\begin{array}{c}\text { Specific } \\
\text { conductance }\end{array}$ \\
\hline \multirow[t]{11}{*}{$(A-11-1) 18 b a a-1$} & $03-01-90$ & 11.0 & 570 \\
\hline & $04-27-90$ & 11.0 & 475 \\
\hline & $05-30-90$ & 11.5 & 430 \\
\hline & $06-28-90$ & 12.0 & 500 \\
\hline & $07-26-90$ & 12.0 & 520 \\
\hline & $08-27-90$ & 12.5 & 485 \\
\hline & $09-27-90$ & 12.0 & 495 \\
\hline & $10-29-90$ & 11.5 & 465 \\
\hline & $11-28-90$ & 10.5 & 455 \\
\hline & $01-17-91$ & 10.0 & 540 \\
\hline & $03-08-91$ & 11.5 & 465 \\
\hline \multirow[t]{11}{*}{ (A-12-1)17daa-1 } & $04-26-90$ & 20.0 & 510 \\
\hline & $05-29-90$ & 20.0 & 500 \\
\hline & $06-28-90$ & 20.5 & 520 \\
\hline & $07-25-90$ & 20.5 & 500 \\
\hline & $08-28-90$ & 20.5 & 500 \\
\hline & $09-26-90$ & 20.5 & 520 \\
\hline & $10-31-90$ & 20.0 & 455 \\
\hline & $11-30-90$ & 20.0 & 470 \\
\hline & $12-20-90$ & 20.0 & 470 \\
\hline & $01-17-91$ & 20.0 & 490 \\
\hline & $03-09-91$ & 20.0 & 490 \\
\hline \multirow[t]{7}{*}{$(A-12-1) 29 c a b-1$} & $06-29-90$ & 15.5 & 520 \\
\hline & $08-28-90$ & 19.0 & 495 \\
\hline & $10-22-90$ & 10.5 & 415 \\
\hline & $10-29-90$ & 10.5 & 415 \\
\hline & $11-28-90$ & 9.5 & 455 \\
\hline & $01-16-91$ & 14.0 & 490 \\
\hline & $03-06-91$ & 16.5 & 500 \\
\hline \multirow[t]{6}{*}{$(A-12-1) 31$ dab-2 } & $03-01-90$ & 16.5 & - \\
\hline & $04-27-90$ & 16.0 & 410 \\
\hline & $10-29-90$ & 16.0 & 440 \\
\hline & $11-28-90$ & 15.5 & 380 \\
\hline & $01-16-91$ & 14.5 & 440 \\
\hline & $03-07-91$ & 14.5 & 415 \\
\hline \multirow[t]{2}{*}{$(A-14-1) 22 b a d-1$} & 03-01-90 & 9.5 & 455 \\
\hline & $04-26-90$ & 10.0 & 460 \\
\hline
\end{tabular}


Table 5.-Temperature and specific conductance of water from selected wells-Continued

\begin{tabular}{|c|c|c|c|}
\hline Location & Date & $\begin{array}{c}\text { Water } \\
\text { temperature }\end{array}$ & $\begin{array}{c}\text { Specific } \\
\text { conductance }\end{array}$ \\
\hline$(A-14-1) 22 b a d-1$-Continued & $\begin{array}{l}05-30-90 \\
06-28-90 \\
07-24-90\end{array}$ & $\begin{array}{l}10.0 \\
10.0 \\
10.0\end{array}$ & $\begin{array}{l}470 \\
475 \\
485\end{array}$ \\
\hline$(B-11-1) 9 c d b-1$ & $\begin{array}{l}03-01-90 \\
04-25-90 \\
05-29-90 \\
06-28-90 \\
07-24-90 \\
08-27-90 \\
09-25-90 \\
10-29-90 \\
11-28-90 \\
01-17-91 \\
03-06-91\end{array}$ & \begin{tabular}{r|}
10.0 \\
11.0 \\
10.0 \\
11.0 \\
11.0 \\
12.0 \\
12.0 \\
11.0 \\
10.0 \\
10.0 \\
9.5
\end{tabular} & $\begin{array}{r}910 \\
950 \\
960 \\
960 \\
1,000 \\
960 \\
980 \\
840 \\
950 \\
950 \\
970\end{array}$ \\
\hline$(B-11-1) 35 c c a-1$ & $\begin{array}{l}03-01-90 \\
04-25-90 \\
05-29-90 \\
06-28-90 \\
07-24-90 \\
08-27-90 \\
09-25-90 \\
10-29-90 \\
11-28-90 \\
01-07-91 \\
03-06-91\end{array}$ & $\begin{array}{l}10.0 \\
11.0 \\
11.0 \\
11.0 \\
11.0 \\
11.5 \\
14.5 \\
13.0 \\
10.5 \\
10.0 \\
10.0\end{array}$ & $\begin{array}{l}730 \\
770 \\
750 \\
780 \\
800 \\
800 \\
800 \\
670 \\
800 \\
770 \\
740\end{array}$ \\
\hline$(B-12-1) 8 c d b-2$ & $\begin{array}{l}03-01-90 \\
04-25-90 \\
05-29-90 \\
06-28-90 \\
07-24-90 \\
08-27-90 \\
09-25-90 \\
10-29-90 \\
11-30-90 \\
01-16-91 \\
03-09-91\end{array}$ & $\begin{array}{l}13.0 \\
13.0 \\
13.5 \\
13.0 \\
13.0 \\
14.5 \\
13.5 \\
12.5 \\
13.0 \\
12.5 \\
12.0\end{array}$ & $\begin{array}{r}730 \\
770 \\
1,200 \\
800 \\
810 \\
770 \\
770 \\
780 \\
750 \\
730 \\
730\end{array}$ \\
\hline$(B-12-1) 15 a d c-1$ & $\begin{array}{l}04-26-90 \\
05-30-90 \\
06-28-90 \\
07-24-90 \\
08-27-90\end{array}$ & $\begin{array}{l}17.5 \\
18.0 \\
18.5 \\
18.0 \\
19.0\end{array}$ & $\begin{array}{l}860 \\
870 \\
880 \\
890 \\
850\end{array}$ \\
\hline
\end{tabular}


Table 5.-Temperature and specific conductance of water from selected wells-Continued

\begin{tabular}{|c|c|c|c|}
\hline Location & Date & $\begin{array}{c}\text { Water } \\
\text { temperature }\end{array}$ & $\begin{array}{c}\text { Specific } \\
\text { conductance }\end{array}$ \\
\hline \multirow[t]{5}{*}{ (B-12-1)15adc-1-Continued } & $09-25-90$ & 19.0 & 860 \\
\hline & $10-29-90$ & 17.5 & 790 \\
\hline & $11-29-90$ & 17.5 & 820 \\
\hline & $01-17-91$ & 17.5 & 830 \\
\hline & $03-07-91$ & 17.0 & 860 \\
\hline \multirow[t]{4}{*}{$(B-12-1) 15 a d c-2$} & $10-29-90$ & 17.5 & 820 \\
\hline & $11-29-90$ & 17.5 & 830 \\
\hline & $01-17-91$ & 17.5 & 840 \\
\hline & $03-07-91$ & 17.0 & 890 \\
\hline \multirow[t]{11}{*}{$(B-12-1) 26 c c a-1$} & $03-01-90$ & 12.0 & 560 \\
\hline & $04-25-90$ & 12.5 & 600 \\
\hline & $05-29-90$ & 12.0 & 600 \\
\hline & $06-28-90$ & 13.0 & 600 \\
\hline & $07-24-90$ & 13.5 & 660 \\
\hline & $08-27-90$ & 13.0 & 650 \\
\hline & $09-25-90$ & 14.0 & 600 \\
\hline & $10-29-90$ & 12.5 & 550 \\
\hline & $11-28-90$ & 12.5 & 560 \\
\hline & $01-16-91$ & 12.0 & 600 \\
\hline & $03-07-91$ & 12.0 & 580 \\
\hline
\end{tabular}




\section{Table 6.-Records of selected springs \\ [ - indicates no data available]}

Location: See page 2 for an explanation of numbering systems for hydrologic-data sites.

Altitude of land surface: In feet above sea level interpolated from U.S. Geological Survey topographic maps.

Probable source of water: Geologic unit from which spring discharges: $\mathrm{Cz} u$, Cenozoic undivided; $\mathrm{pC} u$, Precambrian undivided.

Discharge: gal/min, gallons per minute.

Specific conductance: $\mu \mathrm{S} / \mathrm{cm}$, microsiemens per centimeter at 25 degrees Celsius.

Water temperature: ${ }^{\circ} \mathrm{C}$, degrees Celsius.

\begin{tabular}{|c|c|c|c|c|c|c|c|c|}
\hline \multirow[b]{2}{*}{ Location } & \multirow[b]{2}{*}{ Name } & \multirow{2}{*}{$\begin{array}{c}\text { Altitude } \\
\text { of land } \\
\text { surface } \\
\text { (feet) }\end{array}$} & \multirow{2}{*}{$\begin{array}{l}\text { Probable } \\
\text { source } \\
\text { of water }\end{array}$} & \multicolumn{5}{|c|}{ Field measurements } \\
\hline & & & & $\begin{array}{l}\text { Discharge } \\
\text { (gal/min) }\end{array}$ & $\begin{array}{c}\text { Specific } \\
\text { conductance } \\
(\mu \mathrm{S} / \mathrm{cm})\end{array}$ & $\begin{array}{l}\text { Water } \\
\text { temperature } \\
\left({ }^{\circ} \mathrm{C}\right)\end{array}$ & $\mathrm{pH}$ & Date \\
\hline$(A-10-1) 21$ bab-S1 & - & 4,770 & $\mathrm{Czu}$ & 100 & - & - & - & $06-06-90$ \\
\hline$(A-10-1) 21$ bca-S1 & - & 4,770 & $\mathrm{Czu}$ & 500 & - & - & - & $06-06-90$ \\
\hline \multirow{2}{*}{$(A-10-1) 33$ bac-S1 } & Whites Inc. & 4,820 & $\mathrm{Czu}$ & 76.4 & 530 & 12.0 & 6.4 & $06-06-90$ \\
\hline & & & & 117 & 500 & 9.0 & 7.4 & $12-06-90$ \\
\hline \multirow[t]{2}{*}{$(\mathrm{A}-11-1) 10 \mathrm{ccd}-\mathrm{S} 1$} & Little Ballard Spring & 4,500 & $\mathrm{Czu}$ & 447 & 650 & 12.0 & 6.5 & $05-31-90$ \\
\hline & & & & 277 & - & - & - & $11-21-90$ \\
\hline \multirow[t]{2}{*}{$(A-11-1) 15 b b c-S 1$} & Big Ballard Spring & 4,490 & $\mathrm{Czu}$ & 829 & 600 & 12.0 & 6.2 & $05-31-90$ \\
\hline & & & & 344 & - & - & - & $11-21-90$ \\
\hline \multirow[t]{3}{*}{$(A-11-1) 17 b d b-S 1$} & Spring Creek Number 1 & 4,445 & $\mathrm{Czu}$ & 3,150 & 540 & 14.5 & 6.3 & $06-05-90$ \\
\hline & & & & 2,060 & 670 & 8.0 & 7.9 & $11-28-90$ \\
\hline & & & & 2,050 & 740 & 9.0 & 8.1 & $12-06-90$ \\
\hline \multirow[t]{2}{*}{$(A-11-1) 18 b c d-S 1$} & Spring Creek Number 3 & 4,430 & $\mathrm{Czu}$ & 2,280 & - & - & - & $06-05-90$ \\
\hline & & & & 3,890 & - & - & - & $12-06-90$ \\
\hline \multirow[t]{2}{*}{ (A-11-1) 18bdd-S1 } & Spring Creek Number 2 & 4,430 & $\mathrm{Czu}$ & 2,280 & - & - & - & $06-05-90$ \\
\hline & & & & 3,890 & - & - & - & $12-06-90$ \\
\hline$(A-12-1) 4 b a b-S 1$ & Chambers Spring & 4,470 & $\mathrm{Czu}$ & 421 & 680 & 13.0 & 6.4 & $05-31-90$ \\
\hline \multirow[t]{2}{*}{ (A-13-1) 17dca-S1 } & Joseph Smith Spring & 4,460 & $\mathrm{Czu}$ & 216 & 640 & 11.0 & 6.3 & $06-01-90$ \\
\hline & & & & 165 & 620 & 4.5 & 7.9 & $11-10-90$ \\
\hline \multirow[t]{2}{*}{$(A-13-1) 20 d b b-S 1$} & Corbitt Spring & 4,400 & $\mathrm{Czu}$ & 854 & 510 & 17.5 & 6.5 & $06-07-90$ \\
\hline & & & & 942 & 500 & 8.5 & 7.8 & $11-20-90$ \\
\hline \multirow[t]{2}{*}{$(A-13-1) 20 a b a-S 1$} & William Smith Spring & 4,460 & $\mathrm{Czu}$ & 347 & 590 & 12.0 & 7.8 & $06-01-90$ \\
\hline & & & & 68.2 & 600 & 5.0 & 6.9 & $11-20-90$ \\
\hline \multirow[t]{2}{*}{$(\mathrm{A}-13-1) 20 \mathrm{cca}-\mathrm{S} 1$} & - & 4,440 & $\mathrm{Czu}$ & 600 & 540 & 11.0 & 6.3 & $05-31-90$ \\
\hline & & & & 722 & - & 7.5 & 8.1 & $11-10-90$ \\
\hline \multirow[t]{2}{*}{$(A-13-1) 29 c c d-S 1$} & Gittins Springs & 4,440 & $\mathrm{Czu}$ & 395 & 380 & 14.5 & 6.3 & $05-31-90$ \\
\hline & & & & 503 & 480 & 6.0 & 8.0 & $11-10-90$ \\
\hline \multirow[t]{2}{*}{$(A-13-1) 32 a d c-S 1$} & Hopkins Spring & 4,470 & $\mathrm{Czu}$ & 520 & 640 & 12.0 & 6.4 & $05-31-90$ \\
\hline & & & & 280 & - & - & - & $11-21-90$ \\
\hline \multirow[t]{2}{*}{ (A-14-1)15acb-S1 } & Clear Creek Spring & 4,460 & $\mathrm{Czu}$ & 1,200 & 600 & 12.0 & 6.4 & $06-07-90$ \\
\hline & & & & 552 & 600 & 12.0 & 7.5 & $11-20-90$ \\
\hline \multirow[t]{2}{*}{$(A-14-1) 33 a b b-S 1$} & - & 4,440 & $\mathrm{Czu}$ & 100 & 950 & 4.5 & 8.2 & $06-07-90$ \\
\hline & & & & 2 & - & - & - & $11-10-90$ \\
\hline \multirow[t]{2}{*}{$(A-14-1) 34 b d c-S 1$} & Peart-Thompson & 4,490 & $\mathrm{Czu}$ & 100 & 730 & 10.5 & 6.4 & $06-07-90$ \\
\hline & & & & 30.0 & 650 & 10.0 & 7.2 & $11-20-90$ \\
\hline (A-14-2)29bcd-S1 & Ranger Spring & 5,850 & $\mathrm{pCu}$ & 5,660 & - & - & - & $06-07-90$ \\
\hline (A-14-2)30bba-S1 & Cherry Creek Spring & 5,310 & $\mathrm{Czu}$ & 200 & - & - & - & $06-07-90$ \\
\hline$(B-10-1) 10 a a c-S 1$ & Wellsville Spring & 4,535 & $\mathrm{Czu}$ & 822 & 730 & 18.0 & 6.7 & $06-06-90$ \\
\hline & & & & 930 & 820 & 11.5 & 7.6 & $12-07-90$ \\
\hline$(B-10-1) 10 c a b-S 1$ & Murray Spring & 4,640 & $\mathrm{Czu}$ & 900 & 530 & 11.0 & 6.8 & $06-06-90$ \\
\hline & & & & 1,280 & 495 & 10.0 & 8.2 & $12-07-90$ \\
\hline$(B-11-1) 21 d a c-S 1$ & Gardner Spring & 4,440 & $\mathrm{Czu}$ & 596 & 520 & 11.5 & 6.2 & $06-05-90$ \\
\hline & & & & 1,990 & - & - & - & $12-07-90$ \\
\hline$(B-11-1) 27 d c a-S 1$ & Darley Spring & 4,480 & $\mathrm{Czu}$ & 1,500 & 485 & 9.5 & 8.0 & $12-07-90$ \\
\hline$(B-11-1) 34$ dac-S1 & Northfield Spring & 4,510 & $\mathrm{Czu}$ & 3,200 & 500 & 10.0 & 6.7 & $06-06-90$ \\
\hline & & & & 1,050 & 455 & 10.5 & 7.4 & $11-28-90$ \\
\hline $16 S$ 40E 22DBB1 & Low Spring & 4,800 & $\mathrm{Czu}$ & 11.5 & 405 & 10.5 & 6.3 & $06-08-90$ \\
\hline & & & & 7.3 & 345 & 10.0 & 7.4 & $12-07-90$ \\
\hline
\end{tabular}




\title{
Table 7.-Field measurements of discharge, specific conductance, and water temperature at selected surface-water sites
}

\author{
[- indicates no data available]
}

Location: See page 2 for explanation of the numbering systems for hydrologic-data sites; location numbers are listed in approximate downstream order; letter following location number indicates type of site: B, canal or ditch; W, stream.

Discharge: $\mathrm{ft}^{3} / \mathrm{s}$, cubic feet per second.

Specific conductance: $\mu \mathrm{S} / \mathrm{cm}$, microsiemens per centimeter at 25 degrees Celsius.

Water temperature: ${ }^{\circ} \mathrm{C}$, degrees Celsius.

\begin{tabular}{|c|c|c|c|c|c|}
\hline Location & Date & $\begin{array}{c}\text { Discharge } \\
\left(\mathrm{ft}^{3} / \mathrm{s}\right)\end{array}$ & $\begin{array}{c}\text { Specific } \\
\text { conductance } \\
(\mu \mathrm{S} / \mathrm{cm})\end{array}$ & $\begin{array}{c}\text { Water } \\
\text { temperature } \\
\left({ }^{\circ} \mathrm{C}\right)\end{array}$ & Remarks \\
\hline \multirow[t]{3}{*}{$16 S 40 E$ 04DDC1B } & $08-08-90$ & 30.9 & 310 & 13.5 & \multirow[t]{3}{*}{ Cub River Canal } \\
\hline & $08-09-90$ & 30.6 & 325 & 12.5 & \\
\hline & $08-10-90$ & 29.9 & 320 & 13.0 & \\
\hline \multirow[t]{3}{*}{$16 S 40 E$ 09CBC $1 B$} & $08-08-90$ & 1.11 & - & - & \multirow[t]{3}{*}{ Diversion from Cub River Canal } \\
\hline & $08-09-90$ & 1.27 & - & - & \\
\hline & $08-10-90$ & 1.01 & - & - & \\
\hline \multirow[t]{3}{*}{$16 S 40 E$ 09СBC2B } & $08-08-90$ & 35.4 & 315 & 14.0 & \multirow[t]{3}{*}{ Cub River Canal } \\
\hline & $08-09-90$ & 33.8 & 260 & 13.5 & \\
\hline & $08-10-90$ & 32.2 & 320 & 14.0 & \\
\hline \multirow[t]{3}{*}{$16 S 40 E$ 09СВСзВ } & $08-08-90$ & 4.72 & - & - & \multirow[t]{3}{*}{ Diversion from Cub River Canal } \\
\hline & $08-09-90$ & 2.29 & 258 & 13.5 & \\
\hline & $08-10-90$ & 2.46 & 315 & 14.0 & \\
\hline \multirow[t]{3}{*}{$16 \mathrm{~S} 40 \mathrm{E}$ 08DAB1B } & $08-08-90$ & 3.77 & - & - & \multirow[t]{3}{*}{ Diversion from Cub River Canal } \\
\hline & 08-09-90 & 3.76 & 315 & 14.0 & \\
\hline & $08-10-90$ & 0 & - & - & \\
\hline \multirow[t]{3}{*}{ 16S 40E 08CAA1B } & $08-08-90$ & 1.27 & - & - & \multirow[t]{3}{*}{ Diversion from Cub River Canal } \\
\hline & $08-09-90$ & .904 & 325 & 14.0 & \\
\hline & $08-10-90$ & .706 & 314 & 14.5 & \\
\hline \multirow[t]{3}{*}{ 16S 40E 08BBB1B } & $08-08-90$ & 21.5 & 325 & 15.0 & \multirow[t]{3}{*}{ Cub River Canal } \\
\hline & $08-09-90$ & 21.5 & 330 & 15.0 & \\
\hline & $08-10-90$ & 22.8 & 325 & 15.0 & \\
\hline \multirow[t]{3}{*}{ 16S 40E 07AAA1B } & $08-08-90$ & 1.06 & - & - & \multirow[t]{3}{*}{ Diversion from Cub River Canal } \\
\hline & $08-09-90$ & 1.54 & 330 & 15.0 & \\
\hline & $08-10-90$ & 1.89 & 325 & 14.5 & \\
\hline \multirow[t]{3}{*}{$16 S 40 E$ 06DCB1B } & $08-08-90$ & .134 & 946 & 14.0 & \multirow[t]{3}{*}{ Inflow to Cub River Canal } \\
\hline & $08-09-90$ & .134 & 1,160 & 14.0 & \\
\hline & $08-10-90$ & .134 & 1,160 & 14.0 & \\
\hline \multirow[t]{3}{*}{ 16S 40E 06CAD1B } & $08-08-90$ & .107 & 859 & 15.5 & \multirow[t]{3}{*}{ Inflow to Cub River Canal } \\
\hline & $08-09-90$ & .268 & 1,090 & 15.5 & \\
\hline & $08-10-90$ & .107 & 990 & 14.0 & \\
\hline \multirow[t]{3}{*}{$16 \mathrm{~S} 40 \mathrm{E}$ 06CBB1B } & $08-08-90$ & 23.1 & 325 & 17.0 & \multirow[t]{3}{*}{ Cub River Canal } \\
\hline & $08-09-90$ & 22.1 & 330 & 15.5 & \\
\hline & $08-10-90$ & 25.2 & 335 & 16.0 & \\
\hline
\end{tabular}




\section{Table 7.-Field measurements of discharge, specific conductance, and water temperature at selected surface-water sites-Continued}

\begin{tabular}{|c|c|c|c|c|c|}
\hline Location & Date & $\begin{array}{c}\text { Discharge } \\
\left(\mathrm{ft}^{3} / \mathrm{s}\right)\end{array}$ & $\begin{array}{c}\text { Specific } \\
\text { conductance } \\
(\mu \mathrm{S} / \mathrm{cm})\end{array}$ & $\begin{array}{c}\text { Water } \\
\text { temperature } \\
\left({ }^{\circ} \mathrm{C}\right)\end{array}$ & Remarks \\
\hline 16S 39E 26CBC $1 B$ & $\begin{array}{l}08-08-90 \\
08-09-90 \\
08-10-90\end{array}$ & $\begin{array}{l}68.9 \\
64.4 \\
67.3\end{array}$ & $\begin{array}{l}940 \\
890 \\
900\end{array}$ & $\begin{array}{l}23.0 \\
22.0 \\
21.0\end{array}$ & Cub River Canal \\
\hline 16S 39E 26CCA1B & $\begin{array}{l}08-08-90 \\
08-09-90 \\
08-10-90\end{array}$ & $\begin{array}{c}.447 \\
.447 \\
2.01\end{array}$ & $\frac{-}{-}$ & $\frac{-}{-}$ & Diversion from Cub River Canal \\
\hline 16S 39E 26CDD1B & $\begin{array}{l}08-08-90 \\
08-09-90 \\
08-10-90\end{array}$ & $\begin{array}{l}14.49 \\
14.5 \\
13.9\end{array}$ & - & - & Diversion from Cub River Canal \\
\hline 16S 39E 26CDD2B & $\begin{array}{l}08-08-90 \\
08-09-90 \\
08-10-90\end{array}$ & $\begin{array}{l}1.56 \\
2.24 \\
6.44\end{array}$ & - & $\frac{-}{-}$ & Diversion from Cub River Canal \\
\hline$(A-15-1) 32 c d d-1 B$ & $\begin{array}{l}08-08-90 \\
08-09-90 \\
08-10-90\end{array}$ & $\begin{array}{l}0 \\
1.43 \\
3.34\end{array}$ & $\frac{-}{960}$ & $\frac{-}{22.0}$ & Diversion from Cub River Canal \\
\hline$(A-14-1) 5 a b b-1 B$ & $\begin{array}{l}08-08-90 \\
08-09-90 \\
08-10-90\end{array}$ & $\begin{array}{l}7.60 \\
7.89 \\
8.57\end{array}$ & $\begin{array}{l}838 \\
900 \\
960\end{array}$ & $\begin{array}{l}23.0 \\
23.0 \\
22.0\end{array}$ & Diversion from Cub River Canal \\
\hline$(A-14-1) 5 b d d-1 B$ & $\begin{array}{l}08-08-90 \\
08-09-90 \\
08-10-90\end{array}$ & $\begin{array}{l}6.10 \\
5.81 \\
3.08\end{array}$ & $\begin{array}{l}- \\
925 \\
950\end{array}$ & $\begin{array}{l}- \\
24.0 \\
23.0\end{array}$ & Diversion from Cub River Canal \\
\hline$(A-14-1) 5 b d d-2 B$ & $\begin{array}{l}08-08-90 \\
08-09-90 \\
08-10-90\end{array}$ & $\begin{array}{l}1.70 \\
1.72 \\
2.11\end{array}$ & $\begin{array}{l}- \\
925 \\
950\end{array}$ & $\begin{array}{l}-\overline{24.0} \\
23.0\end{array}$ & Diversion from Cub River Canal \\
\hline$(A-14-1) 5 b d c-1 B$ & $\begin{array}{l}08-08-90 \\
08-09-90 \\
08-10-90\end{array}$ & $\begin{array}{l}2.46 \\
2.40 \\
2.41\end{array}$ & - & $\frac{-}{-}$ & Diversion from Cub River Canal \\
\hline$(A-14-1) 5 c a b-1 B$ & $\begin{array}{l}08-08-90 \\
08-09-90 \\
08-10-90\end{array}$ & $\begin{array}{l}2.57 \\
3.21 \\
2.14\end{array}$ & - & - & Diversion from Cub River Canal \\
\hline$(A-14-1) 5 c d c-1 B$ & $\begin{array}{l}08-08-90 \\
08-09-90 \\
08-10-90\end{array}$ & $\begin{array}{l}46.9 \\
39.7 \\
40.8\end{array}$ & $\begin{array}{l}890 \\
890 \\
990\end{array}$ & $\begin{array}{l}24.0 \\
24.0 \\
22.5\end{array}$ & Cub River Canal \\
\hline$(A-12-1) 14$ baa-1B & $\begin{array}{l}08-22-90 \\
08-22-90\end{array}$ & $\begin{array}{l}25.0 \\
21.7\end{array}$ & $\begin{array}{l}370 \\
355\end{array}$ & $\begin{array}{l}12.5 \\
16.0\end{array}$ & Logan Northern Canal \\
\hline$(A-12-1) 11$ cdd-1B & $\begin{array}{l}08-22-90 \\
08-22-90\end{array}$ & $\begin{array}{l}.109 \\
.030\end{array}$ & $\begin{array}{l}355 \\
345\end{array}$ & $\begin{array}{l}13.0 \\
16.0\end{array}$ & Diversion from Logan Northern Canal \\
\hline$(A-12-1) 11$ codd-2B & $\begin{array}{l}08-22-90 \\
08-22-90\end{array}$ & $\begin{array}{l}.371 \\
.276\end{array}$ & $\begin{array}{l}355 \\
355\end{array}$ & $\begin{array}{l}13.0 \\
16.0\end{array}$ & Diversion from Logan Northern Canal \\
\hline$(A-12-1) 11$ cda-1B & $\begin{array}{l}08-22-90 \\
08-22-90\end{array}$ & $\begin{array}{l}.022 \\
.067\end{array}$ & $\begin{array}{l}350 \\
371\end{array}$ & $\begin{array}{l}14.0 \\
16.0\end{array}$ & Diversion from Logan Northern Canal \\
\hline
\end{tabular}




\section{Table 7.-Field measurements of discharge, specific conductance, and water temperature at selected surface-water sites-Continued}

\begin{tabular}{|c|c|c|c|c|c|}
\hline Location & Date & $\begin{array}{c}\text { Discharge } \\
\left(\mathrm{ft}^{3} / \mathrm{s}\right)\end{array}$ & $\begin{array}{c}\text { Specific } \\
\text { conductance } \\
(\mu \mathrm{S} / \mathrm{cm})\end{array}$ & $\begin{array}{c}\text { Water } \\
\text { temperature } \\
\left({ }^{\circ} \mathrm{C}\right)\end{array}$ & Remarks \\
\hline$(A-12-1) 11 \mathrm{cad}-1 \mathrm{~B}$ & $\begin{array}{l}08-22-90 \\
08-22-90\end{array}$ & $\begin{array}{r}0.007 \\
.033\end{array}$ & - & - & Diversion from Logan Northern Canal \\
\hline$(A-12-1) 11 c a b-1 B$ & $\begin{array}{l}08-22-90 \\
08-22-90\end{array}$ & $\begin{array}{l}24.4 \\
22.7\end{array}$ & $\begin{array}{l}365 \\
356\end{array}$ & $\begin{array}{l}14.5 \\
17.0\end{array}$ & Logan Northern Canal \\
\hline$(A-12-1) 36 b c a-1 W$ & $\begin{array}{l}11-07-90 \\
11-08-90 \\
11-09-90\end{array}$ & $\begin{array}{l}62.3 \\
76.5 \\
62.6\end{array}$ & $\begin{array}{l}215 \\
370 \\
370\end{array}$ & $\begin{array}{l}7.5 \\
3.5 \\
4.5\end{array}$ & Logan River \\
\hline$(A-12-1) 35 a d a-1 B$ & $\begin{array}{l}11-07-90 \\
11-08-90 \\
11-09-90\end{array}$ & $\begin{array}{l}9.54 \\
9.81 \\
9.83\end{array}$ & $\begin{array}{l}- \\
- \\
-\end{array}$ & $\begin{array}{l}- \\
- \\
-\end{array}$ & Inflow to Logan River \\
\hline$(A-12-1) 34 d d d-1 W$ & $\begin{array}{l}11-07-90 \\
11-08-90 \\
11-09-90\end{array}$ & $\begin{array}{l}73.7 \\
82.0 \\
78.9\end{array}$ & $\begin{array}{l}340 \\
365 \\
345\end{array}$ & $\begin{array}{l}4.0 \\
3.5 \\
5.0\end{array}$ & Logan River \\
\hline$(A-10-1) 6 c c c-1 B$ & $08-07-90$ & 48.1 & 460 & 16.0 & Wellsville-Mendon Lower Canal \\
\hline$(B-10-1) 1 d d d-1 B$ & $08-07-90$ & 2.07 & 412 & 21.0 & Diversion from Wellsville-Mendon Lower Canal \\
\hline$(B-10-1) 1 d d c-1 B$ & $08-07-90$ & .882 & 586 & 16.0 & Inflow to Wellsville-Mendon Lower Canal \\
\hline$(B-10-1) 1 d d c-2 B$ & $08-07-90$ & .071 & - & - & Inflow to Wellsville-Mendon Lower Canal \\
\hline$(B-10-1) 1 d d c-3 B$ & $08-07-90$ & .446 & 580 & 15.0 & Diversion from Wellsville-Mendon Lower Canal \\
\hline$(B-10-1) 1 d b c-1 B$ & $08-07-90$ & .396 & - & - & Diversion from Wellsville-Mendon Lower Canal \\
\hline$(8-10-1) 11$ aaa-1B & $08-07-90$ & 1.52 & - & - & Diversion from Wellsville-Mendon Lower Canal \\
\hline$(\mathrm{B}-10-1) 11$ aac-1B & $08-07-90$ & .504 & - & - & Diversion from Wellsville-Mendon Lower Canal \\
\hline$(B-10-1) 11 a d b-1 B$ & $08-07-90$ & 36.1 & 440 & - & Wellsville-Mendon Lower Canal \\
\hline$(B-10-1) 10 d a b-1 B$ & $08-07-90$ & .882 & - & - & Diversion from Wellsville-Mendon Lower Canal \\
\hline$(B-10-1) 10 d b b-1 B$ & $08-07-90$ & 3.16 & 447 & 25.0 & Diversion from Wellsville-Mendon Lower Canal \\
\hline$(B-10-1)$ 10bca-1B & $08-07-90$ & 36.0 & 450 & 23.0 & Wellsville-Mendon Lower Canal \\
\hline$(B-10-1) 3 c b d-1 B$ & $08-07-90$ & 1.14 & - & - & Diversion from Wellsville-Mendon Lower Canal \\
\hline$(B-10-1) 3 c a b-1 B$ & $08-07-90$ & .468 & - & - & Diversion from Wellsville-Mendon Lower Canal \\
\hline$(B-10-1) 3 b d c-1 B$ & $08-07-90$ & 2.30 & - & - & Diversion from Wellsville-Mendon Lower Canal \\
\hline$(B-11-1) 34 c d a-1 B$ & $08-07-90$ & 1.41 & - & - & Diversion from Wellsville-Mendon Lower Canal \\
\hline$(B-11-1) 34 c a b-1 B$ & $08-07-90$ & 19.8 & 450 & 24.0 & Wellsville-Mendon Lower Canal \\
\hline$(B-11-1) 27 c c c-1 B$ & $08-07-90$ & 5.05 & - & - & Diversion from Wellsville-Mendon Lower Canal \\
\hline$(B-11-1) 27 d d a-1 B$ & 08-07-90 & 1.43 & - & - & Diversion from Wellsville-Mendon Lower Canal \\
\hline
\end{tabular}


Table 7.-Field measurements of discharge, specific conductance, and water temperature at selected surface-water sites-Continued

\begin{tabular}{|c|c|c|c|c|c|}
\hline Location & Date & $\begin{array}{c}\text { Discharge } \\
\left(\mathrm{ft}^{3} / \mathrm{s}\right)\end{array}$ & $\begin{array}{c}\text { Specific } \\
\text { conductance } \\
(\mu \mathrm{S} / \mathrm{cm})\end{array}$ & $\begin{array}{c}\text { Water } \\
\text { temperature } \\
\left({ }^{\circ} \mathrm{C}\right)\end{array}$ & Remarks \\
\hline$(B-11-1) 28 a d c-1 B$ & $08-07-90$ & 1.36 & - & - & Diversion from Wellsville-Mendon Lower Canal \\
\hline$(B-11-1) 28 a c c-1 B$ & $08-07-90$ & .042 & - & - & Diversion from Wellsville-Mendon Lower Canal \\
\hline$(B-11-1) 28 b a a-1 B$ & $08-07-90$ & 1.18 & - & - & Diversion from Wellsville-Mendon Lower Canal \\
\hline$(B-11-1) 28 b a a-2 B$ & $08-07-90$ & 14.1 & 440 & 26.0 & Wellsville-Mendon Lower Canal \\
\hline$(A-10-1) 2 d c c-1 W$ & $\begin{array}{l}11-07-90 \\
11-08-90 \\
11-09-90\end{array}$ & $\begin{array}{l}51.4 \\
48.6 \\
52.3\end{array}$ & $\begin{array}{l}400 \\
360 \\
325\end{array}$ & $\begin{array}{l}4.0 \\
5.5 \\
7.5\end{array}$ & Blacksmith Fork \\
\hline$(A-10-1) 2 d c d-1 B$ & $\begin{array}{l}11-07-90 \\
11-08-90 \\
11-09-90\end{array}$ & $\begin{array}{l}3.42 \\
3.51 \\
3.33\end{array}$ & $\begin{array}{l}363 \\
550 \\
560\end{array}$ & $\begin{array}{r}9.5 \\
10.0 \\
10.0\end{array}$ & Inflow to Blacksmith Fork \\
\hline$(A-10-1) 2 c d a-1 B$ & $\begin{array}{l}11-07-90 \\
11-08-90 \\
11-09-90\end{array}$ & $\begin{array}{l}2.24 \\
2.09 \\
2.01\end{array}$ & $\begin{array}{l}\overline{445} \\
445\end{array}$ & $\begin{array}{l}- \\
7.0 \\
8.5\end{array}$ & Diversion from Blacksmith Fork \\
\hline$(A-10-1) 2 \mathrm{cad}-1 \mathrm{~B}$ & $\begin{array}{l}11-07-90 \\
11-08-90 \\
11-09-90\end{array}$ & $\begin{array}{l}5.42 \\
5.33 \\
5.56\end{array}$ & $\begin{array}{l}- \\
450 \\
445\end{array}$ & $\begin{array}{l}- \\
6.5 \\
8.5\end{array}$ & Diversion from Blacksmith Fork \\
\hline$(A-10-1) 2 b c b-1 B$ & $\begin{array}{l}11-07-90 \\
11-08-90 \\
11-09-90\end{array}$ & $\begin{array}{l}1.39 \\
1.39 \\
1.36\end{array}$ & $\begin{array}{l}450 \\
450 \\
300\end{array}$ & $\begin{array}{l}5.5 \\
6.0 \\
7.5\end{array}$ & Diversion from Blacksmith Fork \\
\hline$(A-10-1) 3 a a b-1 B$ & $\begin{array}{l}11-07-90 \\
11-08-90 \\
11-09-90\end{array}$ & $\begin{array}{l}1.18 \\
1.06 \\
1.21\end{array}$ & $\begin{array}{l}- \\
440 \\
440\end{array}$ & $\begin{array}{l}- \\
6.0 \\
8.5\end{array}$ & Diversion from Blacksmith Fork \\
\hline$(A-11-1) 34 d d c-1 B$ & $\begin{array}{l}11-07-90 \\
11-08-90 \\
11-09-90\end{array}$ & $\begin{array}{l}9.32 \\
8.70 \\
8.33\end{array}$ & $\begin{array}{l}445 \\
385 \\
430\end{array}$ & $\begin{array}{l}5.5 \\
6.5 \\
7.0\end{array}$ & Diversion from Blacksmith Fork \\
\hline$(A-11-1) 34 d a c-1 W$ & $\begin{array}{l}11-07-90 \\
11-08-90 \\
11-09-90\end{array}$ & $\begin{array}{l}45.3 \\
41.4 \\
36.6\end{array}$ & $\begin{array}{l}- \\
450 \\
455\end{array}$ & $\begin{array}{l}- \\
6.0 \\
8.5\end{array}$ & Blacksmith Fork \\
\hline$(A-9-1) 10 b b c-1 W$ & $\begin{array}{l}11-07-90 \\
11-08-90 \\
11-09-90\end{array}$ & $\begin{array}{l}12.7 \\
17.0 \\
17.5\end{array}$ & $\begin{array}{l}- \\
445 \\
445\end{array}$ & $\begin{array}{r}- \\
4.5 \\
6.0\end{array}$ & Little Bear River \\
\hline$(A-10-1) 33 b d c-1 W$ & $\begin{array}{l}11-07-90 \\
11-08-90 \\
11-09-90\end{array}$ & $\begin{array}{l}18.2 \\
21.6 \\
21.9\end{array}$ & $\begin{array}{l}- \\
475 \\
471\end{array}$ & $\begin{array}{l}- \\
5.5 \\
7.5\end{array}$ & Little Bear River \\
\hline$(B-11-1) 13 a a b-1 W$ & $\begin{array}{l}08-27-90 \\
09-27-90 \\
11-01-90 \\
11-29-90 \\
01-17-91 \\
03-09-91\end{array}$ & $\begin{array}{l}19.1 \\
22.4 \\
20.6 \\
21.3 \\
22.1 \\
22.2\end{array}$ & $\begin{array}{l}450 \\
550 \\
480 \\
520 \\
580 \\
530\end{array}$ & $\begin{array}{r}17.5 \\
15.5 \\
8.0 \\
5.0 \\
7.0 \\
10.0\end{array}$ & Spring Creek \\
\hline
\end{tabular}


Table 7.-Field measurements of discharge, specific conductance, and water temperature at selected surface-water sites-Continued

\begin{tabular}{|c|c|c|c|c|c|}
\hline Location & Date & $\begin{array}{c}\text { Discharge } \\
\left(\mathrm{ft}^{3} / \mathrm{s}\right)\end{array}$ & $\begin{array}{c}\text { Specific } \\
\text { conductance } \\
(\mu \mathrm{S} / \mathrm{cm})\end{array}$ & $\begin{array}{c}\text { Water } \\
\text { temperature } \\
\left({ }^{\circ} \mathrm{C}\right)\end{array}$ & Remarks \\
\hline (B-11-1)34dac-S1 & $\begin{array}{l}06-06-90 \\
07-26-90 \\
08-27-90 \\
09-25-90 \\
11-06-90 \\
11-28-90 \\
01-17-91 \\
03-09-91\end{array}$ & $\begin{array}{l}7.12 \\
7.07 \\
6.72 \\
2.77 \\
2.54 \\
2.33 \\
1.84 \\
2.32\end{array}$ & $\begin{array}{l}- \\
530 \\
520 \\
540 \\
470 \\
460 \\
590 \\
520\end{array}$ & $\begin{array}{l}- \\
11.0 \\
10.5 \\
11.0 \\
10.5 \\
10.0 \\
10.0 \\
10.0\end{array}$ & Northfield Spring \\
\hline $15 S$ 41E 08ABB1W & $\begin{array}{l}07-31-90 \\
08-28-90 \\
09-26-90 \\
10-31-90 \\
11-29-90\end{array}$ & $\begin{array}{l}40.3 \\
25.8 \\
17.5 \\
13.8 \\
13.4\end{array}$ & $\begin{array}{l}270 \\
230 \\
295 \\
265 \\
306\end{array}$ & $\begin{array}{r}14.5 \\
17.5 \\
13.5 \\
7.0 \\
.5\end{array}$ & Cub River near Franklin, Idaho \\
\hline
\end{tabular}

